\title{
Mediale Zirkulation und Mise en Relation. Relationales Geschichtserleben: Do-it-yourself-History!? (Cluster IV)
}

Die Darstellungen der $\rightarrow$ Kap. 4-6 haben deutlich gemacht, dass aus der von mir entwickelten pragmatischen Perspektive auf digitale Reprisen von Filmen und deren Formen von aisthetischer Historiografie - auch unter der Berücksichtigung der technischen Voraussetzungen von digitalen Bildern $(\rightarrow$ Kap. 2$)-$ Kontextualität und Relativität als grundlegende Prinzipien der Bedeutungsproduktion anzusehen sind. ${ }^{1}$

Dieses $\rightarrow$ Kap. 7 widmet sich jenem wahrnehmungskonfigurierenden und damit auch geschichtsbildmodellierenden Prinzip, welches ich als Mise en Relation bezeichne: Mise en Relation wird als Wahrnehmungsakt, -erfahrung und -haltung angesichts von zeitlichen Differenzen verstanden. Diese Untersuchungsperspektive bindet sich in diesem $\rightarrow$ Kap. 7 an ein spezifisches Phänomen, das dem Film als Medium und Kunstform schon seit seinen Anfängen aus produktions- und distributionstechnischen Gründen innewohnt und heute unter den Vorzeichen der digitalen Domäne eine Aktualisierung mit wahrnehmungstheoretischen Konsequenzen durchläuft: Es geht um die Versionenhaftigkeit filmischer Werke, welche sich vor allem auf deren Reproduzierbarkeit gründet; das Phänomen der Versionenhaftigkeit war schon in der analogen Domäne in Form einer technischen beziehungsweise fotochemisch basierten Reproduzierbarkeit gegeben, in der digitalen Domäne wird es nun umso mehr augenscheinlich.

Zur Erinnerung: Der besondere Ansatz dieser Studie liegt darin, nicht vordringlich den Fokus auf das ontologisch Neue von digitalisierten Filmen $\mathrm{zu}$ richten und dieses bestimmen $\mathrm{zu}$ wollen; vielmehr stehen die Konzeptualisierungen filmischer Wahrnehmungsformen in der Reprise als geschichtsbildmodellierende Prozesse im Zentrum des Interesses. Gegenstand sind insofern konkrete Praktiken und Phänomene der digitalen Reprise, an die nicht allein die Frage gestellt wird, was an ihnen neu ist, sondern darüber hinaus, wie sie uns unter bestimmten Bedingungen vermitteln, dass sie neu seien.

1 Diese Grundsätzlichkeit spiegelt sich in dem etwas größeren Umfang dieses die Fallstudien abschließenden $\rightarrow$ Kap. 7 . 
Mein Ansatz ist nachhaltig geprägt durch ein Geschichtsbild im Zeichen interagierender zeitlicher Schichtungen (methodologisch von mir auch Cluster genannt, vgl. $\rightarrow$ Kap. 3), welches einem medienarchäologischen Prinzip verpflichtet ist. Damit wird das Narrativ eines technologischen, teleologisch ausgerichteten Fortschritts, in dem die Ansicht vertreten wird, die digitale Revolution habe einen Bruch in den Erscheinungs- und Wirkungsformen von Bewegtbildern herbeigeführt, in einer kritischen, zumal pragmatischen Perspektive gesehen.

Heutige Phänomene der Aktualisierung und der Reprise von Filmen in der digitalen Domäne sind in ihrer dynamischen und extrem zeitgebundenen Dimension zu fassen: Sie unterliegen den meist von Marktinteressen geprägten und daher bewusst sehr kurzen Entwicklungszyklen digitaler Technologien, welche ständige Migrationen und Updates er- und einfordern. Auch dies fließt in meine Analyse der erinnerungskulturellen und geschichtsbildmodellierenden Wirkungs- und Wahrnehmungsweisen ein und führt zu der Profilierung der Reprisen als Passagen ( $\rightarrow$ Kap. 3 und $\rightarrow$ Kap. 5). Das zentrale Interesse dieses $\rightarrow$ Kap. 7 gilt vor diesem Hintergrund der Erfahrung der raumzeitlichen Relativität von Filmen, welche - so die paradoxe These dieses Kapitels - insbesondere in der digitalen Domäne zum kulturindustriellen Argument der Konsekration gerät - zum Vehikel der Weihung als Kunstwerk. ${ }^{2}$ Relativität analysiere ich gemäß meinem wahrnehmungstheoretischen Ansatz als Erfahrung beziehungsweise - im Anschluss an $\rightarrow$ Kap. 6 - auch als Form eines Erfahrungskonsums. Vor allem Letzterer zeitigt den Effekt von emotionalen Ein- und Anbindungen, wodurch Erwartungen an den Erinnerungs- wie den Gegenwartswert eines Filmes nachhaltig beeinflusst werden können. Die in diesem $\rightarrow$ Kap. 7 behandelten Ausprägungen von Mise en Relation stellen somit den letzten zu untersuchenden Aspekt von aisthetischer Historiografie und medienhistorisch wirksamer Erfahrungsbildung dar.

Insofern gilt zur generellen Einordnung dieses $\rightarrow$ Kap. 7: Die $\rightarrow$ Kap. 4-6 weisen grundsätzlich eine doppelsträngige Argumentationsstruktur auf, indem sie jeweils a. ausgewählte ästhetische Strategien anhand von Phänomenen digitaler Reprisen herauspräparieren und b. die entsprechenden, auch von kulturindustriellen Kontexten imprägnierten Erfahrungs- und Erwartungshorizonte in Hinblick auf ihre Geschichtsbildmodellierungen dimensionieren. Dies führt überblicksartig zu der - auch methodologisch aufgeschlüsselten Matrix:

2 Vgl. auch unten die Ausführungen zum Begriff der Konsekration bei Bourdieu (2011c). 
$\rightarrow$ Kap. 4: Es werden wechselseitige Bilder im Format Werbeclip in den Blick genommen, die zunächst aus einer zeit- und filmbildtheoretischen Perspektive heraus Ausprägungen und (memo)phänomenale Effekte der Fetischisierung digitaler Reprisen beschreibbar machen.

$\rightarrow$ Kap. 5: Die pragmatische Analyse performativer Aufführungskontexte und Bedeutungsproduktionen von Reprisen frühen Kinos wird verbunden mit einem phänomenologischen, auf die leibliche Gegenwärtigkeit hin ausgelegten Wahrnehmungsmodell - dies seinerseits gekoppelt an die Vorstellung interagierender, differenzieller Zeitschichten: Bridging the gap, marking the difference (Kessler 2011). Die Überlegungen führen zu einer Modellierung des Zuschauers als körperliche (Nach-)Vollzugsinstanz von medial vermittelten Eindrücken von Geschichtlichkeit.

$\rightarrow$ Kap. 6: Hier wird die Legierung der Register von präexistenten, rekontextualisierten Spielfilmbildern und Non-Fiction-Bildern aus semiopragmatischer Perspektive als funktionale Inkorporierung des Imaginären in Geschichtsbildern herauspräpariert und über eine Vorstellung des Prothetischen wiederum an die präsentisch-leibliche Dimension des die Bilder erlebenden Rezipienten geknüpft.

Das vorliegende $\rightarrow$ Kap. 7 untersucht nun vor diesem Hintergrund - wie einleitend ausgeführt - als ästhetische Strategie Formen und Effekte der Mise en Relation verschiedener Filmversionen zueinander. Aufgrund der generellen Bedeutung dieses Prinzips für die bereits genannten Aspekte medienhistoriografischer Erfahrungsbildung hat das $\rightarrow$ Kap. 7 somit synthetisierenden und resümierenden Charakter. In der Analyse konkreter Phänomene greift insofern das $\rightarrow$ Kap. 7 auf die unterschiedlichen Gegenstände der vorherigen Analysekapitel zurück: Sowohl Werbeclips, filmhistorische Dokumentationen und performative Aufführungskontexte als auch Formen der Reprise werden in der Perspektive einer Mise en Relation untersucht.

Inhaltlich ergeben sich damit nachhaltige Ergänzungen zu bereits entworfenen Konzepten. Das Prinzip des Performativen wird - über das bisherige Modell einer filmphänomenologisch verstandenen Interaktion mit Bewegtbildern hinausgehend - nun an einigen Beispielen als tatsächliche körperlich-leibliche Handlungsgeste ausgeführt. ${ }^{3}$

3 Die in $\rightarrow$ Kap. 6 bereits in Aspekten diskutierte Problemstellung der Modellierung des Verhältnisses von (sinnlich) wahrnehmendem Subjekt und Bewegtbildern, welche Geschichte als überzeitliche und unpersönliche Instanz vermitteln, wird in diesem $\rightarrow$ Kap. 7 in einer weiteren Variation aufgegriffen: Auf welche Weise kann man unter diesen Bedingungen eine Form von Do-it-yourself-Geschichte mit Bewegtbildern angehen, wie sie als Phänomen an manchen Konfigurationen zu beobachten ist? 
Funktionale Zusammenhänge des Imaginären und Utopischen ( $\rightarrow$ Kap. 6$)$, die sich mit Formen der Fetischisierung verbinden können ( $\rightarrow$ Kap. 4 ), werden in diesem $\rightarrow$ Kap. 7 um einen weiteren, bisher meist nur implizit benannten Aspekt erweitert: Der Begriff der Konsekration als Weihung in Hinblick auf die Erfahrung eines Kunstwerks schließt an das Modell des Warenfetischismus an. Allerdings werden an dieser Stelle die medialen Erwartungs- und Erfahrungshorizonte um Konzepte des Soziologen Pierre Bourdieu (u. a. 2011c) ergänzt und erweitert: namentlich um die Idee des symbolischen Kapitals, des Prestiges und der kulturellen Autorität. Auf diese Weise werden Ausprägungen der Mise en Relation als Elemente der erlebnisorientierten Vermittlung kultureller Wertigkeiten systematisiert.

Grundsätzlich lassen sich vor diesem Hintergrund meine Darstellungen in den analytischen $\rightarrow$ Kap. 4-7 als Auslegungen von medienhistoriografisch wirksamen Aspekten begreifen, die zusammen ein gemeinsames pragmatisches und phänomenologisches Wahrnehmungsmodell entwerfen. In den einzelnen Kapiteln wird durch die Unterschiede in dem jeweiligen ästhetischen Analyseobjekt, welches jedes für sich andere Aspekte fokussiert, die Aufmerksamkeit auf unterschiedliche funktionale Einordnungen und damit verschiedene Diskurskreise gelenkt. Hier wird deutlich, dass das Problemfeld der digitalen Reprisen nur in einer polyperspektivischen Herangehensweise in seinen zahlreichen Facetten und Implikationen fassbar gemacht werden kann. Entsprechend der Denkfigur eines Clusters sehe ich die jeweiligen Möglichkeiten der theoretischen Auseinandersetzung mit Phänomenen digitaler Reprisen als ein Netz von Begriffen, die zunächst ahierarchisch nebeneinanderstehen und situativ, je nach Kontext, für die analytische Profilierung eines Aspekts aufeinander bezogen werden wollen. Obwohl die in Anschlag gebrachten Ansätze sich zum Teil unterschiedlichen Voraussetzungen verdanken, weisen sie eben doch auch wichtige Schnittmengen auf: Dazu zählen die Betonung performativer Wahrnehmungs- und Sinnbildungsprozesse sowie die wiederkehrende Bedeutung der Wechselwirkungen zwischen Aktuell-Präsentischem und Virtuell-Imaginärem.

In diesem $\rightarrow$ Kap. 7 werden bereits eingeführte grundsätzliche Epistemai der digitalen Domäne (bes. $\rightarrow$ Kap. 1, $\rightarrow$ Kap. $2, \rightarrow$ Kap. 4, $\rightarrow$ Kap. 5) wieder aufgenommen und als Erfahrungs- wie Erwartungshorizonte auf ihre medienhistoriografische Wirksamkeit hin reflektiert. Vor dem Hintergrund der Beschäftigung mit dem Phänomen der Relativität geraten vor allem Begriffe wie Versatilität und Universalität einzelner sinn- und erfahrungskonfigurierender Module in den Blick (u. a. Manovich 2001; Flückiger 2008; Distelmeyer 2012). Dies geht zurück auf die Eigenschaft digitaler Codes des Random Access, die 
Möglichkeit des Direktzugriffs auf einzelne Codes ( $\rightarrow$ Kap. 2). Dieser gilt als Ursprung der mit der digitalen Domäne verbundenen Auflösung von linearen Denkmustern, die in netzwerkartigen Strukturen aufgehen, welche sich etwa durch Hypertextstrukturen mit zahlreichen Links realisieren lassen: Als Flusser die Krise der Linearität (1992/1988) anhand der Fragestellung untersuchte, wie unser Denken, Fühlen, Wünschen, Handeln und Wahrnehmen durch die Struktur von Codes geformt wird, hob er besonders ebenjenes Prinzip hervor, das aus der Möglichkeit des Random Access digitaler Daten entspringt. ${ }^{4}$ Manovich (2001: u. a. 244-285) hat diesen Ansatz mit den Konzepten von Datenbankstrukturen weiter ausgebaut und die dadurch entstehenden Navigationsräume beschrieben. Distelmeyer (2012) greift seinerseits diesen Diskursstrang auf und bezieht das Prinzip der navigierend zu erschließenden Verräumlichung - auch im Vermittlungsmodus einer interaktiven Kartografie - auf die konkreten digitalen Erscheinungsbedingungen von Film in entsprechenden Dispositiven wie Blu-Ray-Disc oder DVD (Distelmeyer 2012: $170 \mathrm{ff}$.$) .$

Diese räumlichen Strukturen als bedeutungsgenerierende sowie als sinnlich erlebte phänomenale Erfahrungshorizonte mit medienhistoriografischer Wirksamkeit zu begreifen, macht den mehrfach hervorgehobenen Ansatz meiner Studie aus. Das vorliegende $\rightarrow$ Kap. 7 richtet dementsprechend seinen Fokus auf Erfahrungen und Haltungen des Vergleichens, in denen AktuellPräsentisches immer in Relation zu etwas Abwesendem - sei es Vergangenes oder eine synchrone, mögliche Variation des Anwesenden - erlebt wird.

4 Zur Digitalisierung als einem mathematischen Abstraktionsprozess von Denken und Wahrnehmung vgl. Flusser (1992: $22 \mathrm{f}$.) respektive Wolf (2000: 23). Letzterer benennt eine „Vermessung der Welt“, aufgrund derer Paradigmen der Effizienz - etwa nach empirisch-mathematisch bestimmbaren Best Practices - maßgeblich an Bedeutung für unsere Weltwahrnehmung gewinnen. Hier spricht Wolf auch von einer Denk- und Wahrnehmungshaltung des Vergleichs (auf der Grundlage abstrakter Daten, unterschiedlicher mathematischer Werte; vgl. $\rightarrow$ Kap. 2). Dies ist nicht zu verwechseln mit meiner Untersuchung des vergleichenden Sehens oder vergleichenden Hörens, bei der es vordringlich um den Einfluss bestimmter dispositivischer, medialer Anordnungen auf die Wahrnehmung von (Film-)Bildern und auf die erkenntnis- und geschichtsbildmodellierende Wirkung geht (vgl. weiter unten $\rightarrow$ Kap. 7.11 und 7.12). 


\section{Digitale Zirkulation. Kunstwert als ästhetischer und dispositivischer Effekt}

Benjamin (1977a/1936) beschreibt im Vorwort zu seinem berühmten KunstwerkAufsatz die dort formulierten Überlegungen als

Thesen über die Entwicklungstendenzen der Kunst unter den gegenwärtigen Produktionsbedingungen. Deren Dialektik macht sich im Überbau nicht weniger bemerkbar als in der Ökonomie. Darum wäre es falsch, den Kampfwert solcher Thesen zu unterschätzen. Sie setzen eine Anzahl überkommener Begriffe - wie Schöpfertum und Genialität, Ewigkeitswert und Geheimnis - beiseite (Vorwort von Benjamin 1977a: 9, Hervorh. FH).

So erklärt Walter Benjamin bereits einleitend zu seinem folgenreichen Aufsatz die Begriffe Schöpfertum, Genialität, Ewigkeitswert und Geheimnis für die Konzeption von Kunst des 2o. Jahrhunderts angesichts der neuen technischen Reproduktions- und Distributionsmöglichkeiten, die der Film bietet, für überkommen.

Das vorliegende $\rightarrow$ Kap. 7 widmet sich an dieser Stelle zunächst der Frage, inwieweit ebendiese von Benjamin für obsolet erklärten Begriffe als dispositivische wie ästhetische Praktiken entgegen der Prognose in der digitalen Domäne weiterbestehen und Erwartungshorizonte wie Erfahrungsräume prägen. Es wird deshalb in den nachfolgenden Überlegungen um die Koexistenz und die Wechselwirkungen verschiedener Konzepte und Praktiken gehen, in denen zum einen Traditionen und Vorstellungen zum Kunstbegriff aus dem 19. wie 20. Jahrhundert weiterbestehen; zum anderen ist dem Fakt Rechnung zu tragen, dass diese immer wieder unter den neuen (Reproduktions-)Bedingungen neu konfiguriert werden müssen. Damit werden in meiner Perspektive kulturelle Wertigkeiten als Effekte von sich nur passagenhaft konstituierenden Relationen entwickelt.

Davon, dass Benjamins Überlegungen angesichts der digitalen Reproduzierbarkeit eine breite aktualisierende Rezeption und Rekonzeptualisierung erfuhren, zeugen zahlreiche Publikationen seit Mitte der 199oer Jahre (vgl. $\rightarrow$ Kap. 2). Insbesondere Manovich (1994), Mitchell (1992), aber vor allem Wolf (2000) und Rodowick $(2007)^{5}$ haben grundsätzlich über die kulturellen Folgen

5 Rodowick (2007) etwa appliziert Nelson Goodmans (1997: 122 ff.) grundlegende Überlegungen zum autografischen und allografischen Kunstwerk vor dem Hintergrund der digitalen Reproduzierbarkeit auf Problemstellungen digitaler Bilder ( $\rightarrow$ Kap. 2): „Computer generated images are not autographic for two reasons: as ,synthetic' images they cannot be considered the physical act of the author's hand, nor do they result in an end product. Indeed one of the great creative powers of digital images is their lack of closure, a quality Philip 
des Prinzips digital nachgedacht und sich auch in den Diskurs um digitale Bilder eingeordnet (vgl. auch Flückiger 2008, vgl. $\rightarrow$ Kap. 2). In seinem Buch Abstracting Reality hat Wolf ein ganzes Kapitel der Frage nach dem Kunstwerk im digitalen Zeitalter gewidmet (2000: $5^{1} \mathrm{ff}$.). Er macht auf das bestehende Paradox aufmerksam, dass gerade die fehlende physische Präsenz, die Opazität und die Notwendigkeit einer technischen Anordnung zur Sicht- und Hörbarmachung des digitalen oder digitalisierten Kunstwerks zu einer Rückkehr der Aura geführt habe (Wolf 2000: 65). Dies bedinge die besondere Bedeutung des Kultwertes, gebunden an Rituale, die sich mehr denn je als kulturelle wie mediale, das heißt auch an Dispositive gekoppelte ästhetische Praktiken manifestieren (vgl. hierzu auch das Ende von $\rightarrow$ Kap. 6). Dies geht einher mit den Möglichkeiten der digitalen Reproduktion, ${ }^{6}$ einer daraus entstehenden Versionenhaftigkeit von Phänomenen und der Distribution. ${ }^{7}$ Insbesondere den Begriff der Zirkulation hat Elsaesser (2002) als synonym mit Digitalisierung identifiziert. Im Folgenden wird mitunter auch der Begriff mediale Migration verwendet, der im gegebenen Kontext analytisch produktiv gemacht wird. ${ }^{8}$ Methodologisch kann dies als eine aktualisierte Auslegung der berühmt gewordenen Fragestellung „Wann ist Kunst?“ von Nelson Goodman (1997) angesehen werden, in deren Kontext in verzeitlichter Perspektive symbolische Funktionen und Rezeptionsweisen als bestimmend für die Einschätzung eines Werkes herausgestellt werden (vgl. hierzu auch $\rightarrow$ Kap. 2).

Die Zirkulation und ständige Re-Kontextualisierung von digitalisierten Kunstwerken bedeutet methodologisch, dass die jeweiligen zeitlichen, das heißt situativen und räumlichen Kontexte eines Phänomens (beziehungsweise eines digitalen, als Kunst identifizierten Werkes) konstitutiv für dessen Wahrnehmungs- und Erfahrungshorizonte werden. Ähnlich argumentiert auch schon Wolf (2000), wenn er "Aura" als Resultat kultureller Praktiken beschreibt. Mit Patrick Frank (1989) erweitert Wolf den Aurabegriff von Benjamin: Aura wird bei Wolf zu einem Produkt, das aus funktionalen Konstellationen von

Rosen has characterized as ,practically infinite manipulability': they are easily reworked, reappropriated, and recontextualized“ (Rodowick 2007: 15). Hervorzuheben bei Rodowick ist an dieser Stelle die Vorstellung der ständigen, bedeutungskonfigurierenden ,Re-Kontextualisierung' eines Kunstwerks in der digitalen Domäne; vgl. hierzu weiter unten die Überlegungen - auch zur Rolle des ,Schöpfers' bei digitalen Re-Editionen.

6 Vermeintlich ohne Generationsverlust, vgl. hierzu die Diskussion bei Flückiger (2008: 46).

7 Schon Mitchell (1992: 53) formuliert dies mit der mehrschichtigen Vorstellung einer grundsätzlichen Unabgeschlossenheit digitaler Codes, manifest etwa bei digitalen Bildern: in deren "mutability and lack of closure, their tendency to proliferate limitless variants“. Diese Qualität bedinge auch die „unkonventionellen Kanäle der Distribution“.

8 Dieser Begriffsgebrauch spielt unter anderem auf den technischen Ablauf des digitalen Datenaustausches an. 
kultureller Autorität und Konsensus resultiert (Wolf 200o:67) $\cdot{ }^{9}$ Meinem Ansatz gemäß werde ich im Folgenden von der Aura als einem (Wahrnehmungs-)Effekt medialer Anordnungen und Vermittlungsformen sprechen. ${ }^{10}$ Ein solcher Effekt ist mit Blick auf meine bisherigen Modellierungen - etwa von historisierenden hin zu digitalisierenden Lektüremodi - auch als auratisierender Lektüre- beziehungsweise Erfahrungsmodus zu begreifen (vgl. insbes. $\rightarrow$ Kap. 3 und $\rightarrow$ Kap 6).

Vor dem Hintergrund der einleitend erwähnten (und von Benjamin für obsolet erklärten) Begriffe hat dies Konsequenzen: Die aufgrund der kollektiven Produktionszusammenhänge sowie der technischen Reproduzierbarkeit ohnehin schon prekäre Frage nach dem Autor eines Films wird nun angesichts der digitalen Domäne noch verkompliziert: „Digital reproduction [...] has also displaced aura onto its creator (the cult of the author or star performer) or its exchange value [...] both of which are enhanced by publicity" (Wolf 2000: 67, Hervorh. FH). Damit hebt Wolf zwei wichtige Aspekte hervor: $\mathrm{Zu}-$ nächst verweist er auf die Tatsache, dass der Begriff eines Schöpfers oder eines verantwortlich zeichnenden Genius als Urheber eines Werkes keineswegs obsolet ist. Vielmehr erlange umso nachhaltiger im Zeitalter der Zirkulation das Singularitätslabel ,Autor' oder ,Starschöpfer/Performer' Bedeutung und kompensiere damit die fehlende physische Einmaligkeit eines Kunstwerks.

Darüber hinaus, und dies stellt den zweiten wesentlichen Punkt dar, wird aufgrund der Zirkulation der Kontext für die Konstitution der jeweiligen kulturellen Autorität (des Schöpfers, des Genius, des verbundenen, nobilitierenden Stars) entscheidend. Das heißt, die kulturelle Autorität muss aufgrund des fehlenden fixen ,Ursprungs', des fehlenden festen Ursprungsortes

Mit Frank (1989) formuliert Wolf (2000: 67): „If we see aura as a by-product of cultural authority rather than as genius, we will see that mechanical reproduction need [sic!] not take away from the aura, but today helps to create it".

10 Im Zusammenhang mit den Epistemai der digitalen Domäne - Abstraktion, Dematerialisierung und Repräsentation - ergeben sich speziell in Fragen der schöpferischen, kreativen Hervorbringung und des Kunstbegriffs nachhaltig konzeptuelle Herausforderungen, da - auch in Erinnerung an Benjamin und die Aspekte der technischen Reproduzierbarkeit - Begriffe von Authentizität und Urheberschaft (s. u.) prekär werden. Hauptreferenz an dieser Stelle, auf die sich Wolf (2000), Rodowick (2007), Flückiger (2008) und Fossati (2009) beziehen, sind die symboltheoretischen Überlegungen des analytischen Philosophen Nelson Goodman ( $\rightarrow$ Kap. 2). Grundsätzlich lässt sich von Goodman (1997) die Vorstellung übernehmen, der zufolge Eigenschaften, welche Kunst und damit auch digitalen Film ausmachen, nicht unabhängig von der jeweiligen Rezeption zu sehen sind. So symbolisiert das Wahrnehmungscluster digitalisierter Film zu unterschiedlichen Zeiten variante Sinnkonfigurationen (vgl. zum Prinzip der Relationen die Ausführungen weiter unten in diesem $\rightarrow$ Kap. 7). 
oder -zeitpunkts angesichts der Zirkulation jeweils über den Kontext konstituiert und (öffentlich wirksam) vermittelt werden. Gerade durch die polymorphe Qualität und die Universalität digitaler Bilder und Kunstwerke (Flückiger 2008) sowie durch die fehlende unmittelbare physische Sichtbarkeit eines ,Werkes' erhalten die kontextuellen und diskursiven Konstitutionsmechanismen eine zentrale Bedeutung. Dies gilt vor allem auch für den wichtigen zum Schöpferkonzept gehörenden Begriff der Einzigartigkeit. Wie im Verlauf dieses Kapitels anhand der Fallanalysen deutlich werden wird, kommen hier rhetorische und wirkungsästhetische Muster zum Einsatz, die gerade im Horizont digitalisierter historischer Filme die Instanz eines neuen schöpferischen „Entdeckers" vermitteln (Bourdieu 2011c: 103; vgl. hierzu weiter unten). Ein anderes Verfahren zur Nobilitierung eines Werkes im Horizont digitaler Zirkulation ist die bewusste künstliche Limitierung des Zugangs zum Werk im Gegensatz zu dessen totaler Verfügbarkeit (Wolf 200o: 69; zu den wahrnehmungstheoretischen Konsequenzen vgl. unten). ${ }^{11}$

Grundsätzlich bedeutet dies, dass die Art und Weise der Ausstellung und Präsentation den kulturellen Wert eines medialen Phänomens konfiguriert. Im Folgenden wird deshalb untersucht, wie über bestimmte dispositivische und ästhetische Verfahrensweisen das digitalisierte Werk und seine Aura als Effekte performt werden. Der Begriff der Performance knüpft hier in mehrfacher Weise an das in $\rightarrow$ Kap. 5 entworfene Modell des Verhältnisses zwischen dem als Ereignis inszenierten ästhetischen Phänomen und dem raumzeitlich phänomenal erlebenden Subjekt an. Das Verhältnis zwischen Phänomen und Subjekt wird als kontinuierlicher, wechselseitiger Austauschprozess gesehen, in dem der Rezipient/Konsument gleichzeitig als Vollzugs- wie Nachvollzugsinstanz funktioniert (dies wird etwa am Modell des oben genannten „Entdeckers" als Element der erfahrenen Konsekration noch entwickelt).

11 Der Konzern Disney liefert in mehrfacher Hinsicht anschauliche Beispiele für die beschriebenen Konsekrationspraktiken. Zunächst hat Disney vor allem seit den frühen 2oooer Jahren immer wieder verschiedene Nobilitierungslabels für Heimkinoeditionen seiner bekanntesten Filme auf DVD und Blu-Ray-Disc entworfen: etwa die PlatinumEdition, die Diamond-Edition oder die Special Collection. Diese Editionen waren immer nur für kurze, sehr begrenzte Zeit auf dem Markt. Auf diese Weise sollte ein hoher Sammler- und auch Marktwert generiert werden. Ähnlich agierte Disney aber auch bereits im Rahmen der Kinodistribution - etwa in der Periode der 1940er bis 196oer Jahre: In regelmäßigen Abständen wurden sogenannte Klassiker wie etwa FANTASIA (1940) immer wieder - zum Teil aktualisiert unter der Maßgabe unterschiedlicher Normierungsprozesse (Begriff nach Garncarz 1992) - ins Kino gebracht und damit bewusst in immer wieder neue Kindheitserinnerungen und Biografien von Generationen als ,Erfahrungsschatz' eingeschrieben. 
Mediale Phänomene der Reprise verstehe ich in diesem Zusammenhang als audiovisuelle präsentische Passagen, die Sinn-und Bedeutungskonfigurationen im Moment ihrer Aufführung vermitteln ( $\rightarrow$ Kap. 3 und $\rightarrow$ Kap. 5). Mögliche historisierende Referenzierungen eines Vorher und Nachher werden damit immer wieder performativ konstituiert und im Somatisch-Sinnlichen erlebt. ${ }^{12}$

In diesem $\rightarrow$ Kap. 7 steht nun die Untersuchung der Erfahrung von Relativität im Mittelpunkt: namentlich die relationale Versionenhaftigkeit des Films als geschichtsbildmodellierende Erfahrung. Der in meiner Argumentation allgegenwärtige kulturindustrielle Kontext wird in $\rightarrow$ Kap. 7 insofern einbezogen, als die Relativität von Filmen als Konsekrationserfahrung verstanden wird. Damit gewinnen die Begriffe Sinn, Bedeutung, Prestige und kulturelle Autorität eine prozessuale und verzeitlichte Qualität. Das heißt, sie befinden sich in einem ständigen Wandel, sie sind abhängig von den jeweiligen (medialen) Bedingungen und Anordnungen und werden von dem Rezipienten/Nutzer als sinnliche Eindrücke (nach)vollzogen.

Darüber hinaus bindet ebendiese Form der prozessualen Bedeutungskonstitution den Rezipienten beziehungsweise Konsumenten in den Prozess ein - etwa im Moment der situativ geforderten Auswahl einer Filmversion. ${ }^{13}$ Dies wird durch digitale Dispositive begünstigt oder gar initiiert. Allerdings, und das ist einer der spezifischen Effekte, geschieht dies immer im Bewusstsein der (virtuellen) Koexistenz einer alternativen Möglichkeit. Auch hier kommt die charakteristische performative Rezeptionshaltung und sinnliche Erlebnisform zur Geltung.

12 Hier wird noch einmal meine Abgrenzung zu anderen Studien in diesem Gegenstandsbereich deutlich, denen es im Anschluss an Bourdieu (2011a/1971; 2011b/1972; 2011c; 2011d/1994) um die Aufschlüsselung der Verantwortlichkeiten der beteiligten Akteure, Institutionen und Instanzen der Konsekrationsakte geht - eine Perspektive, wie sie etwa Fossati (2009) einnimmt; wie vielfach betont, stehen bei mir die medialen Phänomene und ihre wirkungsästhetische Dimension im analytischen Fokus; vgl. weiterführend zu der praktischen, netzwerkartigen Interaktion verschiedener Instanzen und Institutionen in der Filmkonservierung und -Re-Edition Heller/Flückiger (2010), Cherchi Usai (2012) sowie Flückiger/Heller/Op den Kamp et al. (2016) bzw. Gracy (2007).

13 Vor diesem Hintergrund wird in $\rightarrow$ Kap. 7 nach den digitalen Konfigurationen gefragt, in denen über verschiedene Symbolsysteme und deren innere Relationen Versionen von Geschichts- und damit Weltmodellierungen vermittelt werden. Auch wenn ich dies in meinem phänomenologisch imprägnierten Ansatz nicht grundlegend systematisch übernehme, so könnte man weiterführend die Funktionsweisen eines kognitiven ,Weltenbaus‘ von medialen Konfigurationen mit Nelson Goodman (1990) beschreibbar machen: Komposition/Dekomposition, Gewichtung, Ordnen, Tilgung und Ergänzung, Deformation. 


\subsection{Die Relativierung von Prestige und kultureller Autorität als Erfahrungsmodus}

Konsekration als wesentlichen Teil der Erfahrung eines Werks in den Blick zu nehmen, schließt in mehrfacher Hinsicht an meine Überlegungen zum Warenfetischismus in $\rightarrow$ Kap. 4 an. In der Folge wird über den Rekurs auf die Begrifflichkeit von Pierre Bourdieu (2011a; 2011b; 2011c; 2011d) die Idee des symbolischen Kapitals, des Prestiges und der kulturellen Autorität - ergänzend zu den Überlegungen in Kap. 4 zum Fetischismus - als Erwartungs- und Erfahrungshorizont angesichts von Ausprägungen der Mise en Relation diskutiert. Auf diese Weise sollen auch Aspekte eines kulturellen,Werte'begriffs in mein Modell der aisthetischen Geschichtsbildmodellierung digitaler Reprisen erweiternd integriert werden. In dem Zusammenhang werden einige zentrale strukturelle Befunde von Bourdieu aufgegriffen, die sich - abweichend von Bourdieus ursprünglich kultursoziologischem Kontext - in einer wirkungsästhetischen Analyse digitaler Reprisen produktiv machen lassen.

Im Feld der kulturellen Produktion geht es nach Bourdieu (etwa 2011c) vor allem um die Akkumulation von symbolischem Kapital. Allerdings unterliege die Akkumulation von Kapital - im Gegensatz zu anderen Feldern der Produktion - im kulturellen Feld spezifischen Leugnungen und damit Formierungen der Wahrnehmung: Da der Kunsthandel (respektive die Kulturindustrie, FH) per se mit Dingen handle, die nicht handelbar seien, lasse sich in diesem Feld oberflächlich die Logik einer vorkapitalistischen Ökonomie verfolgen. ${ }^{14}$ Das bedeute, dass im Kontext der Vermarktung von Kultur der ökonomische Kontext geleugnet und vordergründig dem Kommerz eine Absage erteilt werde (Bourdieu 2011c: 98 ). ${ }^{15}$ Stattdessen stünden die Akkumulation von „Prestige“ und „Autorität" als Ziel und Währung im Mittelpunkt, die dann - so Bourdieus kultursoziologischer Befund - allerdings ihrerseits als symbolisches Kapital fungierten und nicht minder in tatsächlichen Gewinn umgesetzt werden könnten (Hafke 2012). Bourdieu bezeichnet dies als „Konsekrationskapital, das die Macht beinhaltet, Dingen (durch Marken- oder Signatureffekt) oder Personen (durch Veröffentlichung, Ausstellung usw.) die Konsekration und damit einen Wert zu verleihen“" (Bourdieu 2011c: 99).

\footnotetext{
14 Da die Praktiken im kulturellen Feld „als praktische Leugnungen [der ökonomischen Ziele, FH] funktionieren, kann man nur tun, was man tut, indem man so tut, als täte man es nicht" (Bourdieu 2011c: 98).

15 Die Leugnung der ökonomischen Interessen im Feld der kulturellen Produktion bezeichnet Bourdieu als mauvaise foi („schlechter Glaube“, hier übersetzt mit „Unaufrichtigkeit“ [Bourdieu 2011c: 100 f.]). In den weiteren Ausführungen beschreibt Bourdieu diese Zusammenhänge als eine in sich sehr widersprüchliche „ideologische Maske“.
} 
In meiner wahrnehmungstheoretisch ausgerichteten Untersuchung werden die genannten Bourdieu'schen Aspekte des symbolischen Kapitals als erfahrenes Kapital verstanden. Dies schließt wiederum an die Analysen einer Warenästhetik digitaler Reprisen ( $\rightarrow$ Kap. 4 ) an, bei der Gebrauchswertversprechen in den ästhetischen Erlebnisversprechen liegen. Dies führt weiter zu der Idee eines imaginär emotional bindenden Erfahrungskonsums angesichts von ästhetischen Objekten als Waren ( $\rightarrow$ Kap. 6$)$.

In Anknüpfung an diese bereits genannten kulturindustriell geprägten Wahrnehmungsmodi aus $\rightarrow$ Kap. 4 und $\rightarrow$ Kap. 6 werden Prestige und kulturelle Autorität als mediale Erwartungs- und Erfahrungshorizonte perspektiviert, die - um mit meinem Gegenstand zu argumentieren - unsere Erlebnisse von digitalisierten Filmen konfigurieren. Da im vorliegenden $\rightarrow$ Kap. 7 die Versionenhaftigkeit von Filmen im Mittelpunkt der Analyse steht, sind insofern Horizonte von Prestige und kultureller Autorität in ihrer Funktionalisierung in den entsprechenden relationalen, verhältnissetzenden Konfigurationen zu untersuchen.

Bourdieus sprechender Titel seines zentralen Textes (2011c) weist in diesem Zusammenhang auf die besondere Bedeutung der Erwartungshaltung des Rezipienten beziehungsweise Konsumenten hin: Die Produktion des Glaubens ist Dreh- und Angelpunkt für die Art und Weise, wie wir etwas als bedeutsames Kunstwerk (ästhetisch) erleben. Daher spielen - aus meiner Perspektive - die Erwartungshaltungen sowie die Wahrnehmungsmodi für die Vermittlung des kulturellen Wertes eine wesentliche Rolle. Dies knüpft in gewisser Weise an meine Überlegungen in $\rightarrow$ Kap. 4 an, denen zufolge H. Böhme zum Fetischismus eine ähnliche Wahrnehmungshaltung entwarf: Ein Fetisch kann nur dann seine Wirkung als solcher entfalten, wenn man daran glaubt. In meinem Ansatz gilt dies umso mehr für die digitale Qualität von Bildern (vgl. $\rightarrow$ Kap. 4): Die digitale Qualität eines Filmes ist nie das, was von sich aus immer schon digital ist, sondern es muss der Glaube als spezifischer Wahrnehmungsmodus erzeugt werden, dass dem so sei (F. Heller mit H. Böhme 2012: 370).

Bourdieus Konzepte und H. Böhmes Verständnis vom Fetischismus haben insofern eine gemeinsame Schnittmenge, als sie die (Wahrnehmungs-)Haltung des Rezipienten und Konsumenten in die Bestimmung eines Kunstwerkes und des Wertes einbeziehen.

Dennoch sind auch die diskursiven Differenzen in ihren Nuancen zu berücksichtigen. Der Begriff des Fetischismus ist vor allem psychoanalytisch und triebstrukturell besetzt, da er Verschiebungsmechanismen und Aspekte der Ersatzbefriedigung thematisiert. Im medialen (Wahrnehmungs-)Kontext führt dies vor allem zur Verhandlung von voyeuristischer Schaulust. Insofern 
vermitteln fetischisierende mediale Strukturen Erwartungs- und Erfahrungshaltungen der Bedürfnisbefriedigung.

Die zitierten Modelle des Soziologen Bourdieu operieren hingegen im Bereich der kritischen Analyse des Feldes der Kunstproduktion. Die sozialen Praktiken werden auf ihre hegemonialen Geltungsansprüche hin untersucht. Damit ergänzen Bourdieus Ausführungen an dieser Stelle meine Überlegungen in $\rightarrow$ Kap. 4 um Aspekte der Bedeutung der dispositivischen und ästhetischen Inszenierung von kultureller Autorität für das Erlebnis eines Filmwerkes in der digitalen Reprise.

Insbesondere hier wird, mit Bourdieu (2011c: 101) gesprochen, eine „Ideologie des Charismas“ wirksam. Die „Ideologie des Charismas“ - etwa das eines genialen Schöpfers - sei der eigentliche Ursprung des Glaubens an den Wert des Kunstwerks (Bourdieu 2011c: 101). ${ }^{16}$ So gelte es, in der Zirkulation von ästhetischen Objekten kritisch das omnipräsente Konzept des Autors als Schöpfer zu hinterfragen: Es gehe darum, die Faktoren, die eine Instanz im Kontext eines Werkes „autorisieren“, kritisch in den Blick zu nehmen (Bourdieu 2011c: 101). ${ }^{17}$ Bourdieu hat an dieser Stelle namentlich Kunsthändler und Verleger und deren gesellschaftliche Rolle im Blick.

Gerade bei der digitalen Zirkulation und Re-Edition von Filmen lassen sich diese Überlegungen produktiv adaptieren. Der anschlussfähige Grundgedanke liegt darin, dass das Werk eines Schöpfers von jemand anderem als Handelsware verwendet wird. Bei der Vermarktung des Werkes eines anderen

16 Diese „Ideologie des Charismas“, die auf den einen kreativen Schöpfer des Kunstwerks zielt, stellt sich beim Film doppelt kompliziert dar, da er von vornherein das Ergebnis gemeinschaftlicher und industrieller Zusammenhänge ist.

17 Hier ließe sich fruchtbar ein weiteres Diskursfeld zur partizipatorischen Medienkultur insbesondere auch mit Blick auf die digitale Medienumgebung - anschließen: Der vor allem von Henry Jenkins (1992) unter dem Begriff „textual poachers“ geprägte Ansatz, Fankulturen und die daraus resultierenden medialen Praktiken zu untersuchen, hat vielfach Resonanz in Studien zu einer partizipatorischen Digitalkultur gefunden. In einer solchen Perspektive wird die Entscheidungs- und Handlungsmacht der Produktionsseite der Medienindustrie über Angebote und Produkte relativiert, indem sie nicht mehr als alleinig bestimmende Faktoren gesehen werden. Vielmehr werden Fankulturen als auf die Medienindustrie Einfluss nehmende, produktive Akteure herausgestellt; etwa indem sie selbst zu (Ko-)Autoren von zirkulierenden audiovisuellen Paratexten, Fortschreibungen und Kommentaren werden (zum hier in Anschlag gebrachten Begriff des Produsers vgl. später weiterführende Anmerkungen). Nichtsdestotrotz hat H. Jenkins ausdrücklich darauf hingewiesen, dass ein Fanpublikum nicht automatisch gleichzusetzen sei mit einem (inter),aktiven Zuschauer“. Aus einer jüngeren Perspektive hat etwa Einwächter (2014) H. Jenkins und seine Interpreten auf ihre rhetorischen und methodischen Muster hin überblicksartig aufgearbeitet (2014: $158 \mathrm{f}$.). 
wird aber gerade das „Heilige“ des Werkes (Bourdieu 2011c: 101), das sich durch den ursprünglichen Autorenmythos konstituiert, instrumentalisiert und rekonfiguriert. Dies verbindet sich mit dem Narrativ eines „Entdeckers“, der ein Werk, das andernfalls im Zustand einer natürlichen und ungenutzten Ressource geblieben wäre, „zu ,entdecken' verstanden hat, indem er es durch eine Ausstellung, Veröffentlichung oder Inszenierung auf den Markt bringt" (Bourdieu 2011c: 101). Solche Verfahrensweisen fasst Bourdieu als Konsekrationsakte, die sich in der Interaktion verschiedener Akteure im Feld der kulturellen Produktion entfalten und wirksam werden.

Im Horizont meiner Fragestellungen rücken aber, anders als bei Bourdieu, die erinnerungskulturellen und geschichtsbildmodellierenden Konsequenzen in den Fokus, welche in einem besonderen Spannungsfeld stehen. Spezifische Widersprüchlichkeiten sind nicht zu übersehen: Auf der einen Seite geraten Konsekrationsakte als pragmatisch operierende Prozesse in den Blick, die mit Eindrücken von Prestige und kultureller Autorität den Glauben an ein auratisches, einzigartiges Kunstwerk vermitteln; auf der anderen Seite entfaltet sich die paradoxe Situation, dass die Konsekration eines Filmwerkes sich gerade des Reichtums seiner koexistierenden Versionen und Variationen verdankt. Dies hat auf Seiten des Zuschauers eine Wahrnehmungsdisposition zur Voraussetzung wie zur Folge, in der ständig der Glaube an eine imaginär präsente Alternative - eine mögliche andere Version - evoziert und als Erwartungshaltung eingefordert wird. Nicht mehr nur die singuläre Fassung eines Werkes zieht die Aufmerksamkeit auf sich, sondern das clusterförmige Ensemble an (auswählbaren) Varianten begründet den Eindruck von kultureller Werthaltigkeit. Selbst das ästhetische Vergnügen basiert zu einem beträchtlichen Teil auf der Mise en Relation und Differenzqualität der verschiedenen Versionen. Dies wird insbesondere in den Auswirkungen auf die Wahrnehmung konkreter Filme und ihrer diegetischen Universen zu untersuchen sein.

Nicht zuletzt sei in diesem Kontext des vorliegenden $\rightarrow$ Kap. 7 auf zwei weitere Widersprüche dieses Diskurskreises mit Blick auf die digitale Domäne hingewiesen: Ähnlich, wie es H. Böhme schon für den Fetisch beschrieb, gilt schon grundsätzlich bei Benjamin (1977a) eine bestimmte raumzeitliche Anordnung als notwendige Voraussetzung, um ein auratisches Kunstwerk als solches zu erleben:

Die Definition der Aura als ,einmalige Erscheinung einer Ferne, so nah sie sein mag‘, stellt nichts anderes dar als die Formulierung des Kultwerts des Kunstwerks in Kategorien der raum-zeitlichen Wahrnehmung. Ferne ist das Gegenteil von Nähe. Das wesentlich Ferne ist das Unnahbare. In der Tat ist Unnahbarkeit eine Hauptqualität des Kultbildes (Benjamin 1977a: 14, Fußnote 7). 
Wie bereits in $\rightarrow$ Kap. 4 entwickelt, bedeutet dies zum einen, dass der Glaube an ein auratisches Phänomen durch raumzeitliche Unerreichbarkeit situativ etabliert wird; zum anderen bedingt dies gleichzeitig einen Wahrnehmungsmodus (beim Fetisch noch deutlicher um den Aspekt der sexuellen Lust erweitert), der über die Performanz der Überhöhung den Rezipienten/Konsumenten als Teil des Prozesses benötigt.

So findet man im Kontext der digitalen Distributionsformen in unterschiedlichen Variationen den Topos der Aneignung (Klinger 2006a; Wortmann 2010; Distelmeyer 2012; F. Heller 2013a): Insbesondere bei digitalen Dispositiven wie DVD und Blu-Ray-Disc wird die Suggestion von possessiver Nähe zu Filmen im privaten Bereich hervorgehoben. Video-on-Demand stellt hierbei vor dem Hintergrund der suggerierten Personalisierung des Angebots durch Algorithmen, welche die Auswahl des sichtbaren Menüs strukturiert, eine besonders spannungsgeladene, zugespitzte Variante dar (Keiper 2014b).

Im Folgenden geht es deshalb um Erfahrungen von Relativität im Horizont potenziell unbegrenzter, allgegenwärtiger Zugriffsmöglichkeiten, Aneignungsversprechen und auratisierender Ferne. Die jeweiligen ästhetischen wie dispositivischen Konfigurationen spielen eine zentrale Rolle für geschichtsbildmodellierende Prozesse.

Zu der beschriebenen räumlichen Spannung gesellt sich in der analytischen Betrachtung auch eine zeitliche. H. Böhme weist auf das kulturpolitische Konstrukt von Ewigkeit hin - gerade wenn es um den musealen und erinnerungskulturellen Bereich geht:

Wir [H. Böhme] glauben, dass die Gesellschaften, je mehr sie ins Zeichen neoliberaler Globalisierung und der digitalen Immaterialität treten, desto stärker jener zeitstillenden Zonen bedürfen, in denen die Dinge [...] eine Sphäre der Zeitlosigkeit erhalten, die der Tauschsphäre entgegengesetzt ist, umzirkelt vom Noli me tangere der musealen Ordnung. Die gesammelten Dinge stellen die Transzendenz der Moderne dar, die annahm, Transzendenz entbehren zu können (H. Böhme 2011: 369-370).

In diesem von H. Böhme entworfenen grundsätzlicheren Kontext erhält die Konstitution von „zeitstillenden Zonen“ anthropologische und auch kulturpolitische Bedeutung. Aus wahrnehmungstheoretischer Perspektive mit Blick auf den Film impliziert die Vorstellung von "zeitstillenden Zonen“ einen besonderen Erfahrungshorizont, der sich zwischen den Polen der Erinnerung an Vergangenes und der dem Medium eigenen präsentischen Eindrücklichkeit aufspannt.

Vor diesem Hintergrund sind auch die Implikationen von Barbara Klingers (2006a) Aussage genauer zu perspektivieren, wenn sie schon für den 
amerikanischen TV-Kabelsender AMC befindet: „The channel asserts the value of its artifacts as timeless classics" (Klinger 2006a: 99, Hervorh. FH). Deshalb geht es nachstehend darum, die Erfahrung von Relativität in digitalen Reprisen in aller Widersprüchlichkeit aufzuspüren und (kultur- und memopolitisch) einzuordnen.

\section{7·3 Mise en Relation als Verfahren der Konsekration}

Im Zusammenhang mit heutigen Ausprägungen der Mise en Relation im Zeichen digitaler Dispositive ist einführend ein kurzer Exkurs zur generellen Versionenhaftigkeit von Film vonnöten - insbesondere auch in der Ausweisung der Konsequenzen. Stefan Drößler, Filmhistoriker und Direktor des Filmmuseums München, merkte 2004 eindrücklich an, dass die Film- respektive Medienwissenschaft bisher kaum Kenntnis davon genommen habe, dass das Material, mit dem sie arbeite, sich durch ständig wandelnde technische und dadurch auch ästhetische Bedingungen in der Zeit verändere. Diese grundsätzliche Beobachtung hat nachhaltige Folgen für (bestimmte) filmanalytische Fragestellungen. Es wird zunehmend schwierig, einen singulären, Referenztext ${ }^{\star}$ zu bestimmen. Die Präsenz von Filmen auf verschiedensten digitalen Plattformen und Speichermedien schafft immer neue Kontexte und Anordnungen, die - so meine These zur Wirkungsästhetik dieses Phänomens - eine spezifische mediale Erwartungshaltung über Prozesse der Mise en Relation kreieren. Insbesondere mit dem Vertrieb von filmhistorischem Material über digitale Dispositive und digitale Derivate (unterschiedliche Ausgabeformate) potenziert sich die bisherige Problematik der Filmfassungen als vermeintlich verlässliche Referenz (der Forschung) enorm. Es gibt in wissenschaftlichen Zusammenhängen meines Erachtens aktuell kaum das Bewusstsein für die Disparität digitaler Filmquellen. Dies wiegt umso schwerer - und das ist ja der Fokus dieser Studie und insbesondere des vorliegenden $\rightarrow$ Kap. $7_{- \text {, als }}$ die Relativität und Versatilität von filmischen Texten und Werken auch auf phänomenaler Ebene nachhaltige Konsequenzen zeitigen.

Das Phänomen der Versionenhaftigkeit von Filmen - Chris Wahl (2009) spricht auch von einer "generellen Versionenhaftigkeit des Films" (C. Wahl 2009: 10, Hervorh. FH) - existiert seit Beginn der Film- und Kinogeschichte. Zum einen sind unterschiedliche Fassungen ${ }^{18}$ eines Films ein Produktionseffekt (nach C. Wahl auch Präversionen). So war es etwa insbesondere in der

18 Zum Begriff der Fassung vgl. grundsätzlich Garncarz (1992): „Filmfassungen sind Ergebnisse von Variationsprozessen, in denen Filme signifikant variiert werden“ (1992: 13). 
früheren Phase der Filmproduktion bis in die 1920er Jahre hinein gängige Praxis, gleichzeitig mit mehreren Kameras zu drehen, um mehr Kameranegative für Kopien, auch für den Export, zur Verfügung zu haben (s. unten auch das Beispiel Die NibeLungen). ${ }^{19}$ Gleichzeitig entstanden verschiedene Fassungen ebenso als Distributionseffekt (C. Wahl nennt es unter anderem Postversionen): In unterschiedlichen geografischen Regionen wurden und werden andere technologische, kulturelle, moralische, ideologische, ästhetische Normierungsprozesse wirksam, an die der jeweilige Film von verschiedenen Instanzen und Akteuren („Spezialisten“) angepasst wird (Garncarz 1992: 18 ff.).

Im vorliegenden Kontext sind vor allem die Formatfassungen/-versionen (zur Begriffsunterscheidung vgl. C. Wahl 2009: 26) interessant, die technologischen Normierungsprozessen unterliegen - etwa in der Migration eines Films aus dem Kino in das Fernsehen, auf eine DVD oder in das Angebot eines Streamingdienstleisters. Inwieweit solche technologischen Normierungen neue Formatversionen kreieren können, ${ }^{20}$ die signifikant auch ästhetischen Variationen unterworfen werden, habe ich in $\rightarrow$ Kap. 2 anhand der Praxis der Colorization im amerikanischen Fernsehen der 198oer Jahre mit seinen erinnerungspolitischen Konsequenzen für das kulturelle Gedächtnis diskutiert. ${ }^{21}$ Ein ähnliches Verfahren der Veränderung von Filmwerken im Horizont der Verschiebung der Aufführungsdispositive - vom Kino als Ort der Filmaufführung hin zur Ausstrahlung im TV - wäre das Pan-und-Scan-Verfahren ( $\rightarrow$ Kap. 2).

Das Verhältnis zweier Fassungen oder Versionen zueinander bestimmt Garncarz (1992) mittels der Überprüfung des sogenannten Konstanzverhältnisses - wie hoch oder wie niedrig jeweils die Summe an Gemeinsamkeiten oder Differenzen zwischen den jeweiligen Filmen einzuschätzen ist (1992: 15).

Der Weltverband der Filmarchive FIAF ${ }^{22}$ hielt 2013 zu dem Problemkomplex sein jährliches Symposion unter dem Titel Multiversions ab - unter anderem weil die Existenz von verschiedenen Versionen eines Films eine Herausforderung sowohl für die bibliothekarisch-archivarische Erfassung als auch für die Sicherung darstellt. Außerdem ist das Phänomen von hoher Relevanz für Restaurierungen und Rekonstruktionen, die in ihrem jeweiligen

19 Garncarz (1992: 16) und C. Wahl (2009: 27) nennen 1914 als Startdatum der Praxis, Berriatúa (zit. n. Riambau 2013: 96) kommt zu einem anderen Ergebnis: „[F]ilming with more than one camera was widespread throughout the entire silent period“.

$20 \quad$ Hier wird Garncarz' Begriffsgebrauch im Kontext von Filmfassungen (1992) modifiziert.

21 Zur Frage, inwieweit die Praxis der Colorization auch als juristische Normierungspraxis verstanden werden kann, vgl. Op den Kamp (2018).

$22 \quad$ FIAF = Fédération Internationale des Archives du Film. Weitere Informationen zur hier zitierten Konferenz sind zu finden unter http://www.fiafcongress.org/2013/programa. php?lang=en (15.07.2016). 
Arbeitsprozess zwangsläufig eine Referenz für die Fassung, die sie wiederherstellen wollen, wählen müssen. Oft führen die Überlieferung und damit das Vorhandensein von verschiedenen Kopien und Fassungen, das heißt von unterschiedlichen Quellmaterialien, zu synthetischen, hypothetischen Restaurierungen (vgl. Bohn 2013b: 399 ff.). Solche Restaurierungen entstehen auch unter dem Narrativ der ,komplettesten' Fassung; eine solche wird dabei aus verschiedenen überlieferten Filmkopien, die unter Umständen auf ästhetischer und dramaturgischer Ebene variieren, hergestellt.

Das Vorhandensein von Versionen offenbart grundsätzlich eine Flexibilität des (historischen) Films - ob ästhetisch oder textuell auf der Storyebene. In digitalen Distributionskanälen können weitere Versionen eines Werkes in neuen Relationen ein ökonomisches sowie sinnliches Mehrwertversprechen darstellen. Wie in den theoretischen Vorbemerkungen dargelegt, werde ich dies als Teil einer Konsekration untersuchen, die sich in das mediale Erlebnis des Zuschauers - auch verstanden als Nutzer - übersetzt.

Riambau (2013: 97) schreibt im Vorwort der Begleitpublikation zum FIAFKongress, dass das Aufkommen der DVD den Zugang zu verschiedenen Versionen desselben Films oder zu zusätzlichem Material (Outtakes, alternative Enden) multipliziert habe. Das hier wohl populärkulturell bekannteste Phänomen von Film-Re-Editionen - die Vermarktung als Director's Cut impliziert ideell diskursive Verschiebungen: Das Label Director's Cut bekräftigt, so Riambaus Beobachtung, den moralischen Sieg über die vom Produzenten auferlegten Zwänge. Damit - so meine Formulierung - spielt das Label mit der Freisetzung einer konsekrativ wirksamen Autorenintention.

Interessant ist an Riambaus Aussage nicht nur die Tatsache, dass er den $\mathrm{Zu}-$ sammenhang zwischen der medienhistorischen Versionenhaftigkeit des Films generell und der Entwicklung der Laserdisc respektive später der DVD und der Blu-Ray-Disc deutlich macht. Darüber hinaus schließt Riambau gedanklich an meine These an, die einleitend in der Auseinandersetzung mit Benjamin zur Stellung des Kunstwerks im 20. Jahrhundert formuliert wurde: Riambau beschreibt die Bedeutung des Autorenkonzepts als wichtiges Narrativ für die Bestimmung des einen Films, dessen Autor/Regisseur als ,Schöpfer 'über das Dispositiv DVD mit den Möglichkeiten der Kontextualisierung durch audiovisuelle Paratexte eine Möglichkeit erhält, ,selbst zu Wort zu kommen‘ (am bekanntesten ist hier wohl der Fall von Blade Runner [1982], s. u.). Dass die DVD respektive die Blu-Ray-Disc hier als Trägermedium mit seiner Speicherkapazität - und damit mit der Möglichkeit, verschiedene Versionen gleichzeitig präsentieren zu können - eine widersprüchliche Rolle als Dispositiv von Reprisen spielt, wird im Folgenden an den Beispielen illustriert.

Diese Anordnung hat aber auch grundsätzliche Konsequenzen für die Wahrnehmung des editierten Films als Werk: Wenn Jan Distelmeyer (2012) 
seine umfassende Studie dem Dispositiv der DVD und Blu-Ray-Disc unter dem Titel Das flexible Kino widmet, so richtet er vor allem den Blick auf die Flexibilität der Filme im Kontext der genannten Dispositive. Distelmeyer hebt an dieser Stelle die oft mit editierten Outtakes und Deleted Scenes/Nicht verwendete $(n)$ Szenen hervor, die über DVDs beziehungsweise Blu-Ray-Discs zugänglich gemacht werden. Diese seien "das wichtigste Extra, ein Herzstück der weiteren Existenz des Films auf DVD“ (Zion 2002, zit. n. Distelmeyer 2012: 9o). In solchen Anordnungen sei das, was als Film auf der DVD oder Blu-Ray-Disc erscheine, Verhandlungs- und Entscheidungssache des Users. Dies verbindet Distelmeyer unter Rekurs auf Gwóźdź (2009) mit dem Begriff der Selektivität (Distelmeyer 2012: 140). Konzeptionell habe solch rahmendes Material für die Filmwahrnehmung zur Folge, dass die Versatilität des Filmtextes sichtbar werde. Gerade bei Editionen von als älter empfundenen Filmen werde mit geschichtlich argumentierenden Paratexten (Bonusmaterialien etwa in Form von filmhistorischen Dokumentationen) die Wandelbarkeit von Filmen zu unterschiedlichen Zeitpunkten ausgestellt. Die Möglichkeit der Koexistenz verschiedener Versionen auf unterschiedlichen Plattformen oder gar zusammen auf einem zirkulierenden Träger wie der DVD oder Blu-Ray-Disc hat - so Riambaus Formulierungen (2013: 97 f.) zugespitzt gewendet - auch historiografische Folgen, denn die Existenz verschiedener Versionen affiziere den Filmhistoriker. Nicht nur müsse man darauf achten, welche Version vorliege, über die man schreibe. Darüber hinaus sei die daraus entstehende zeitliche Komplexität der Zusammenhänge als eine Topografie, als ein Netzwerk zu begreifen, das schon lange aufgehört habe, linear zu sein (Riambau 2013: 97).

Angesichts der Koexistenz von Versionen verdichtet sich so unter den neuen Re-Editions-, Präsentations-, Zirkulations-, Zugangs- und Rezeptionsbedingungen die Vorstellung einer Filmgeschichtsschreibung hin zu einer Kartografie, in der die einzelnen Elemente - gerade angesichts der extrem kurzen Entwicklungszyklen digitaler Medientechnologien - sich nur momentan in einer Relation zueinander (im Raum) konstituieren. In einer solchen Konfiguration koexistieren alternative Möglichkeiten eines Filmwerkes nebeneinander. Dies erinnert an Thomas Elsaessers Modell (2004) einer medienarchäologischen Perspektive auf filmhistorische Phänomene, wenn er von einer Vergangenheit unter vielen anderen möglichen Vergangenheiten spricht. Schon in $\rightarrow$ Kap. 3 wurde Elsaessers methodologisches Modell auf die wahrnehmungstheoretische Ebene übertragen: Angesichts digitalisierter Filme sowie ihrer digitalen Zugänglichkeit und Zirkulation spreche ich von einer Medienarchäologie im Modus der medialen Erfahrung. Das vorliegende $\rightarrow$ Kap. 7 setzt dies um, indem es konkrete Varianten der Mise en Relation einzelner Phänomene in den Blick nimmt und nach den Dimensionen medialer Erfahrungen fragt, die als historiografisch wirksam begriffen werden. 
Will man in diesem Kontext zudem die Konsekrationsmechanismen, etwa die Konstruktions- und Vermittlungsformen von kultureller Autorität, die hier manifest werden, analysieren, so wird der Begriff der relationalen Rhetorik, wie sie Distelmeyer mit Manovich (2001) der DVD zuordnet, wichtig (Distelmeyer 2012: 113). ${ }^{23}$ Die Effekte des Relationalen sind im wirkungsästhetischen Horizont zu untersuchen. Zur Erinnerung: Casetti (2010) hat für die Rezeptionsseite angesichts digitaler Dispositive die Begriffe relationales Tun und Performance durch den Rezipienten als aktiv Handelnden und Nutzenden ins Spiel gebracht $(\rightarrow$ Kap. 5). Dies bringe ich im weiteren Argumentationsverlauf dieses $\rightarrow$ Kap. 7 mit wissenschaftshistorischen und erkenntnistheoretischen Überlegungen aus der Kunstgeschichte zum vergleichenden Sehen zusammen; namentlich mit Positionen von Heinrich Dilly (1995), der seinerseits grundsätzliche Überlegungen Heinrich Wölfflins aufgreift und aktualisiert. Alle diese Ebenen sind in den Untersuchungen einer Mise en Relation zu berücksichtigen.

Als weitere methodologische Einordnung sei an dieser Stelle kurz abschließend noch darauf hingewiesen, dass diese Herangehensweise im Anschluss an Kesslers historische Pragmatik (u. a. Kessler 2002b; Kessler 2010), die er auch am Fassungsphänomen des Films erläutert (Kessler 2002a), als digitale Pragmatik begriffen werden kann. Dies bindet sich, wie auch schon einleitend zu diesem $\rightarrow$ Kap. 7 dargestellt, in mehrfacher Hinsicht an technische Voraussetzungen und daraus entstehende Epistemai der digitalen Domäne zurück: so etwa die mehrschichtige „Unabgeschlossenheit“ digitaler Codes und Bilder nach Mitchell (1992: 53), die in deren "mutability and lack of closure, their tendency to proliferate limitless variants" zu suchen ist. Gerade aufgrund der polymorphen Qualität und der Universalität digitaler Bilder und Kunstwerke (Flückiger 2008) sowie infolge der fehlenden unmittelbaren physischen Sichtbarkeit (= Opazität) eines ,Werkes', dessen Sichtbarmachung immer erst eines (technischen und kulturellen) Interpretationsvorgangs bedarf, kommt den kontextuellen und diskursiven Konstitutionsmechanismen zentrale Bedeutung zu. Hier wird das grundlegende Epistem der digitalen Domäne verräumlichter Netzwerkstrukturen virulent, die aus dem bereits thematisierten Random Access, der Möglichkeit des Direktzugriffs auf einzelne Codes und Module, resultieren ( $\rightarrow$ Kap. 2 ). Dieser gilt als Ursprung der mit der digitalen Domäne verbundenen Auflösung von linearen Denkmustern, die in netzwerkartigen Strukturen aufgehen, welche sich etwa durch Hypertextstrukturen mit zahlreichen Links realisieren lassen. Zur Erinnerung: Distelmeyer (2012) hat mit Blick auf Erscheinungsformen von Filmen in

23 Vgl. hierzu auch die Analysen zur ästhetischen Vermittlung des Verhältnisses von alt und neu in den Überlegungen des $\rightarrow$ Kap. 4. 
digitalen Dispositiven mit Manovich (2001) in diesem Kontext die Vorstellung von Navigationsräumen entwickelt.

\subsection{Konsekration des Vergleichs. CHANGE THE WAY TO WATCH MOVIES. EDITOR'S GUIDE (2O12)}

In der Annahme, dass es gerade in der digitalen Re-Edition von Filmen zu einer verstärkten Wiederaufnahme von Konsekrationsmechanismen kommt, die eigentlich von Benjamin für obsolet erklärt wurden, richten die folgenden Überlegungen den Blick auf ein Beispiel, in dem gerade das Prinzip des ,Entdeckens' eines Geheimnisses sowie das Spiel mit Nähe und Ferne als wahrnehmungsästhetische Vermittlungsmodi zur Anwendung kommen.

Wie bereits in den Ausführungen in $\rightarrow$ Kap. 4 am Exempel der medienhistoriografisch wirksamen Erfahrungsbildung in Form von Fetischisierung dargelegt, basiert die Aushandlung und Vermittlung der Differenz von alt und neu auf ästhetischen Verfahren, die gemäß einer relationalen Rhetorik funktionieren und zudem eine spezifische Erlebnisform schaffen; das heißt, es wird momenthaft, im Augenblick, das Erlebnis einer zeitlichen Differenz kreiert. Allerdings ist diese relationale Rhetorik in der Vermittlung von sogenannten Entwicklungssprüngen vergleichsweise unabhängig von den jeweiligen tatsächlichen Medientechnologien, die hier in ein Verhältnis gesetzt werden. $^{24}$

Im Folgenden ist es wichtig, die Produktion des Glaubens auf die Neuheit hin, die in diesem Kontext noch widersprüchlicher zu sein scheint, kritisch zu beleuchten. Ein Widerspruch ist bereits offensichtlich: In der Denotation möglicher Qualitäten der Blu-Ray-Disc lassen sich neuere Eigenschaften des Trägers erkennen, die aber mit bekannten ästhetischen Mitteln, Topoi und Motiven beworben werden. Der Glaube an die Neuheit wird in einem - etwa durch Attraktionsmontage - auf Überwältigung angelegten filmischen Erlebnis vermittelt $(\rightarrow$ Kap. 4$)$.

Das hier besprochene Beispiel ergänzt insofern die entsprechenden Analysen, die im Kontext von ästhetischen Strategien der Fetischisierung in $\rightarrow$ Kap. 4 vorgenommen wurden, nun mit dem Fokus auf der Vermittlung von Relativität als Konsekrationserfahrung.

24 Die rhetorischen wie filmisch-formalästhetischen Muster zur Promotion einer DVD unterscheiden sich grundsätzlich in entsprechenden Clips wenig von denen zur Blu-RayDisc oder zum VoD. Über digitale Speichermedien hinaus lassen sich ähnliche rhetorische Figuren und Motive auch durch die ganze Bildmediengeschichte verfolgen; vgl. ausführlicher $\rightarrow$ Kap. 4 . 
Bei dem Untersuchungsgegenstand handelt sich um einen Trailer für BluRay-Discs als Speichermedium von Sony aus dem Jahre 2012, einen Clip, dessen Struktur und Form sich in zahlreichen ähnlichen Werbefilmen auf YouTube wiederfinden lässt. ${ }^{25}$ Hier werden die bereits im $\rightarrow$ Kap. 4 beschriebenen Verfahren um das Ausstellen einer besonderen Mise en Relation anschaulich erweitert. Erneut steht am Anfang die im Vergleich zu bisherigen Seherfahrungen differenzielle Rezeptions- und Erlebnisform von Filmen durch diesen digitalen Träger als Dispositiv: „Sony Pictures and Blu-ray-Disc are changing the way you watch movies"; diese grafische Tagline wird als sich aufbauende Schrifteinblendung zudem von einer autoritären männlichen Off-Stimme, die diesen Satz ausruft, affirmiert.

Es folgt die bekannte formalästhetische Überlegenheitsrhetorik in Hinblick auf die Auflösung und Wiedergabequalität in Bild und Ton: „Look for these High Definition movies on Blu-ray“. Und auch der Mythos der Interaktivität wird beschworen - interessanterweise immer noch durch die zwischenzeitlich schon selbstverständliche alineare, sprunghafte Anwählbarkeit von Kapiteln und die Möglichkeit des Vor- und Zurückgehens im zeitlichen Verlauf des Films. Mit der Einführung des Videorecorders und der VHS-Kassette wurde dieser Entwicklung Vorschub geleistet, spätestens mit der Durchsetzung der DVD etablierten sich solche alinearen Zugriffsmodi als normalisierte Rezeptionsformen (Corrigan 1991; Hickethier 1995; Zielinski 2003; Distelmeyer 2012). Wichtig für den folgenden Zusammenhang ist das Versprechen einer Blu-Ray-Disc, das fiktionale Universum eines Films um weitere räumlich immersive Dimensionen zu erweitern (Distelmeyer 2012: $162 \mathrm{ff}$.); ein Versprechen, das suggeriert, man könnte Zusatzfunktionen (Special Features) mit erweiterndem (Meta-)Wissen über den Film rezipieren, „without ever to leave the movie“. Visuell wird dies mit extrahierten und damit stillgestellten, verfügbar gemachten Standbildern von Hauptfiguren aus dem beworbenen Film umgesetzt. Die Verräumlichung zielt auch auf die Gestaltung des Menüs als Navigationsraum, der die Bedingung definiert, wie man den Film und sein Universum ,betritt'.

Besonders deutlich wird die Überlagerung von Fiktion, beworbener Technologie und der auf Einbindung des Nutzers/Users ausgelegten Mise en Relation in dem Teil des Clips, in dem die Re-Edition von CLOSE ENCOUNTERS OF THE THIRD KIND (Spielberg, 1977) vorgestellt wird. Der Titel und das Thema des Films - die Begegnung mit extraterrestrischen Lebensformen, die alles verändert - wird auf die Rezeptionsform übertragen. Die Blu-Ray-Disc mit ihren Features ermögliche eine noch nähere Erfahrung des Films in der

25 Change the way to watch movies. Editor's guide (2012). Sony Pictures: https:// www.youtube.com/watch?v=16h56tTC $47 \mathrm{M}(17.07 .2016) \rightarrow$ Filmverzeichnis. 
Rezeption im heimischen Raum: "Get close and personal with CLOSE ENCOUNTERS OF THE THIRD KIND“ (Hervorh. FH). Diesmal werden (im Unterschied zu den Clips, die in $\rightarrow$ Kap. 4 besprochen wurden) Fragmente aus dem Film eingeführt und kombiniert mit einer kleinen Abbildung der Verpackung der Blu-Ray-Disc mit dem zeitgenössischen Filmplakat auf dem Cover unten rechts im Bild: Fiktionales Universum und die neue Zugangsform sowie zeitgenössische Paratexte (Filmplakat) werden symbolisch zusammen in einem Bild vereint. Das Versprechen eines Eintretens in eine neue Wahrnehmungsdimension des Films, der selbst die Geschichte einer Veränderung der Weltwahrnehmung erzählt, wird über die zitierten Filmfragmente vermittelt: Türen werden geöffnet, in Großaufnahmen sieht man Figuren aus dem Film überrascht die Augen weiten (Abb. 7.1-7.2).

Abb. 7.1

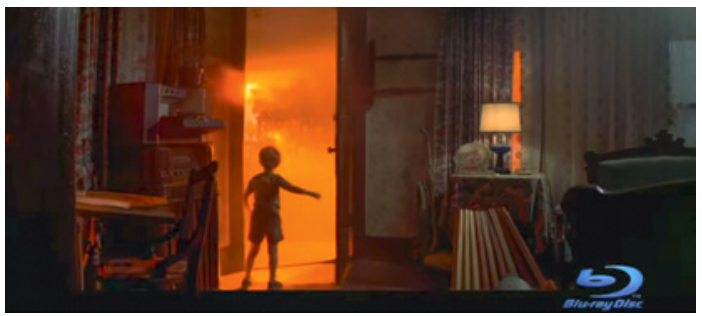

Abb. 7.2

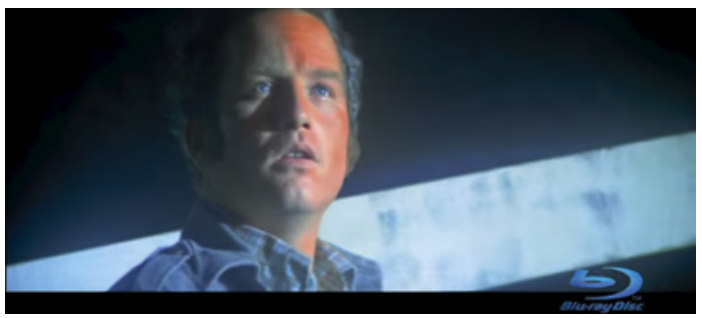

Die Re-Edition dieses Films und seine Fassungsgeschichte ${ }^{26}$ werden mit der neuen Speicherkapazität der Blu-Ray-Disc verbunden - unter dem Titel des neuen Features eines EdITOR'S GUIDE: „With Blu-ray's unmatched storage

26 Es gibt mittlerweile drei Versionen des Films: 1977 erschien der sogenannte Theatrical Cut, der damals in die Kinos mit einer Dauer von 135 Minuten kam. 1980 drehte Steven Spielberg selbst Material nach und schnitt den Film um. Die sogenannte Special Edition kam mit 132 Minuten Länge in demselben Jahr erneut in die Kinos und wurde Grundlage für den ersten Home Video Release. Diese Version war bis 1998 die einzige, die über den HomeVideo-Markt im Umlauf war. 1998 schnitt Steven Spielberg den Film erneut um. Diese Schnittversion erschien in jenem Jahr unter dem Label Director's Cut (Foster 2007). Alle drei Versionen wurden dann 2007 auf einer Blu-Ray-Disc als 3oth Anniversary Ultimate Edition wieder veröffentlicht. Diese Edition wird nun hier in dem Clip beworben. 
capacity see all three versions on a sci-fi classic on a single disc“. Zu diesem aus dem Off gesprochenen (Werbe-)Text sieht man ein Menü, das in kleine Bildschirme zerlegt ist, die wiederum ein großes Bild zusammensetzen. Die einzelnen Fenster blinken unterschiedlich in verschiedenen Farben auf und zitieren damit die in dem Film gezeigte Lichtorgel, die das Kommunikationsinstrument mit den Außerirdischen darstellt. Darunter befindet sich eine beschriftete Menüleiste: „Play Movie - Director's Cut - Special EDition - OriginAL VERSION" (Abb. 7.3).

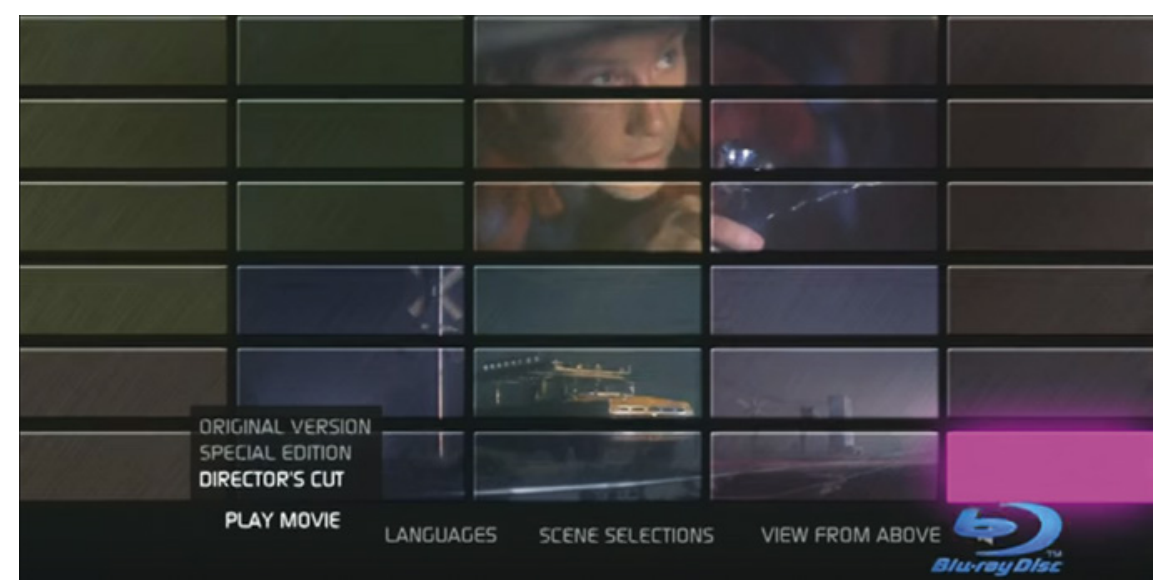

Abb. $7 \cdot 3$

Dann folgen wieder Filmfragmente, der Ausschnitt eines Dialogs aus dem Film ist zu hören: „You recently had a close encounter.“ - „I wanna speak to someone in charge!“. Der aus der Diegese des Films entnommene Dialogfetzen überträgt sich in der Montage auf die sich nun eröffnenden Möglichkeiten der zukünftigen Nutzung durch einen Zuschauer/Konsumenten: Dieser habe so die assoziativ vermittelte Botschaft des Clips - mit dem neuen Träger die Möglichkeit, eine Erfahrung zu machen, die seine Wahrnehmung verändert. Der Blu-Ray-Disc-Nutzer könne die Kontrolle übernehmen. Die akusmatische Voice-Over des Clips fährt suggestiv fort: Man könne einen „View from above“ einnehmen in dem sogenannten EDITOR's GUIDE. Vor den blinkenden einzelnen Fenstern der Lichtorgel erscheinen nun bei der Anwahl des entsprechenden Features in der Menüzeile darunter drei nebeneinanderstehende Textblöcke, die die jeweilige Einstellung in Textform verorten:

Activating View from Aвove provides In-Movie-Pop-Up Graphics which point out differences in the Special Edition and Director's Cut, as compared to the Original Version of the film. Look for the icons below as you are watching the version of your choice (Hervorh. FH). 
Der Vergleich, so die Darstellung in dem Clip, funktioniert über Texttafeln, die jeweils Zusatzinformationen zu der gezeigten Szene enthalten - die Szene in Beziehung setzend zu den anderen auf der Disc enthaltenen Versionen: "This scene/shot does not appear in the original version" oder "the original version contains a scene which is not in the currently viewed version (a short scene description of the missing scene is provided)“ (Abb. 7.4-7.5).

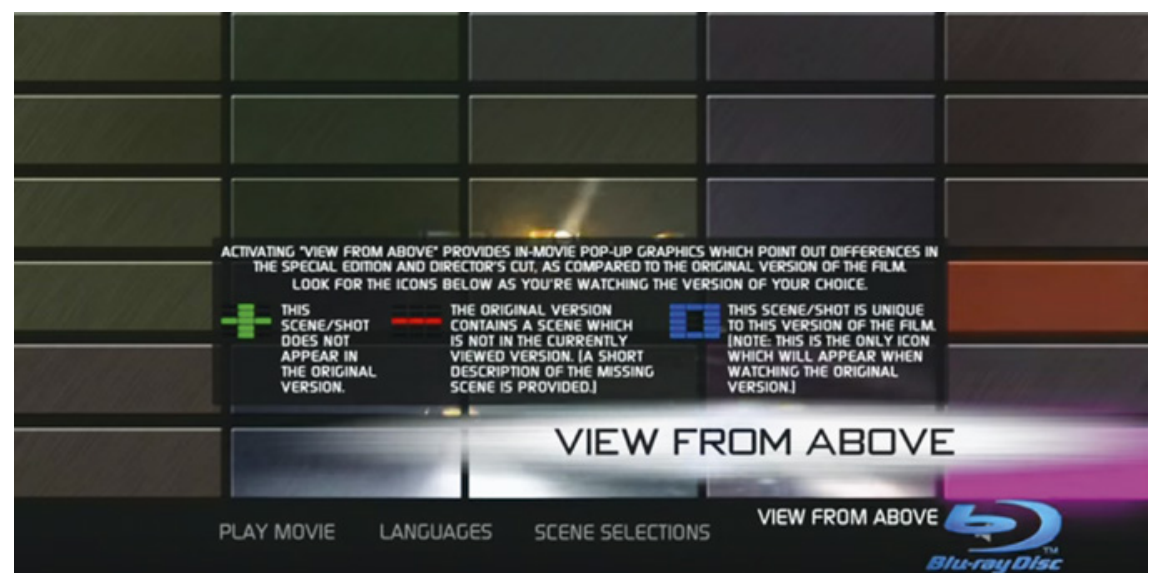

Abb. $7 \cdot 4$

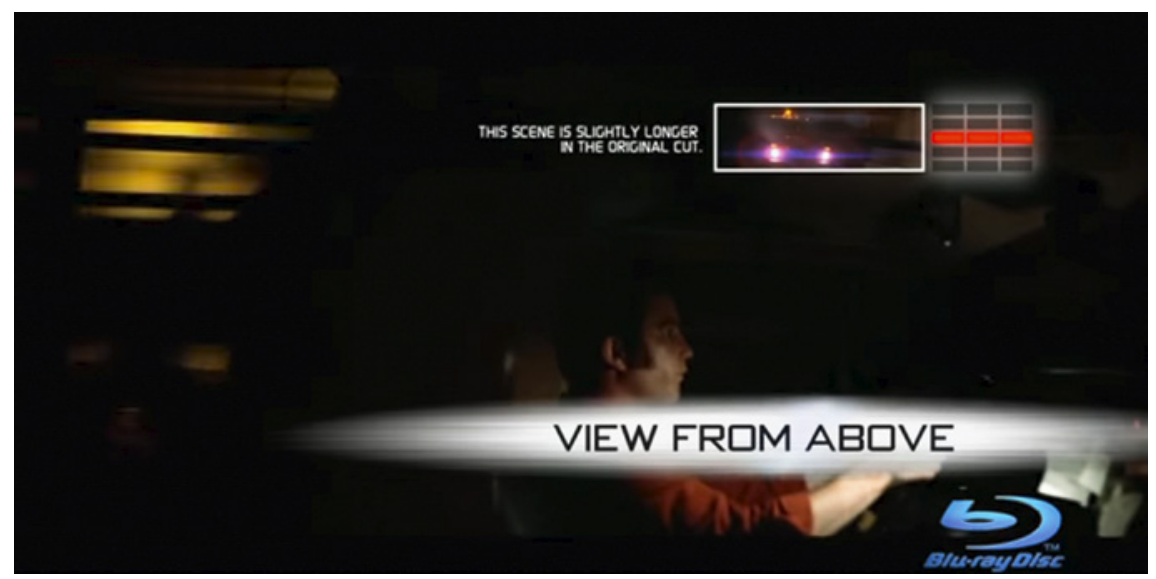

Abb. $7 \cdot 5$

Das Beispiel changiert zwischen Auratisierung und digitalen performativen Aneignungsversprechen. Der hier dispositivisch angepriesene Mehrwert der Blu-Ray-Disc findet sich in der Verheißung auf die Einbindung des $\mathrm{Zu}-$ schauers über den Modus des Vergleichens: Nicht nur das Filmerlebnis steht 
im Mittelpunkt, sondern das differenzielle Erlebnis dreier Versionen - angepriesen als seinerseits neue Wahrnehmungsform, die weniger quellenphilologisch reflektierend wirkt, als vielmehr ein auf Variation aufbauendes vertieftes Eintauchen in das Universum der Fiktion verspricht; die Fiktion ist nicht mehr allein die lineare Erzählung, sondern umfasst nun einen ganzen Erlebnisraum des Vergleichs von narrativen und atmosphärischen Konstanten und Variationen. Dadurch erhält der Film als verlässliches Werk und als Text einen prekären Status - und dies, obwohl er von der Off-Stimme gleichzeitig als "Sci-Fi-Classic" tituliert wird und damit diskursiv einen überzeitlichen ,Ewigkeitswert' zugeschrieben bekommt. Allerdings - und dies ist eine neue (paradoxe) Wendung - lässt sich nun die Erfahrung, dass es verschiedene historische Versionen des Films gibt, als sinnliche Vermittlung der Konsekrationserfahrung begreifen, die sich an der Relativität des Filmwerkes und dessen Erfahrung festmacht.

Für die Wahrnehmung der Filmerzählung in einer Version bedeutet dies: Sie ist immer eine der möglichen Optionen, es könnte auch anders sein. Damit erhält der Film als Werk und als diegetisches Universum etwas Mystisches, das sich dem Zuschauer, immer über das Aktuelle hinausweisend, entzieht. Der Zuschauer kann zwar mit der Blu-Ray-Disc zum Entdecker des Geheimnisses des filmischen Universums werden: „Versions of your choice“; das heißt, zum einen wird Nähe mit der geheimnisvollen filmischen Story versprochen. Aber gleichzeitig - durch den ständig möglichen alternativen Filmtext - verweist die Koexistenz der Versionen immer über die aktuelle Wahl hinaus und erweitert das fiktive Universum in einer Projektion auf dieses unbestimmte Mehr. Gerade in der ständigen Präsenz der Möglichkeit einer imaginären Alternative - in der Relativierung des aktuell präsenten diegetischen und ästhetischen Gefüges vermittelt sich die Erfahrung einer nach wie vor bestehenden Unerreichbarkeit. Hier zeigt sich das Paradox in Benjamins Theorem, wenn man es auf aktuelle digitale Distributions- und Zirkulationsprozesse anwendet: Gerade das Erlebnis der Versionenhaftigkeit trägt zur Auratisierung von Filmen in der digitalen Reprise bei.

Es fällt auf, dass der Clip, ähnlich wie die bereits in den Analysen in $\rightarrow$ Kap. 4 besprochenen Beispiele, insbesondere mit fragmentarischen Filmausschnitten arbeitet, die Superhelden oder Mutanten zitieren. Die narrativen Implikationen einer solchen Bebilderung der Qualitäten einer Blu-Ray-Disc verweisen somit erneut auf das Versprechen einer neuen Wahrnehmungsform, die das menschliche Wahrnehmungsvermögen übersteigt und transformiert. Es zeigt sich das Versprechen der Transzendierung des eigenen Körpers - zunächst über die sinnliche Erfahrung eines Films auf Blu-Ray-Disc. Daneben kommt aber eine weitere Ebene hinzu: Dem Zuschauer als aktivem Nutzer wird suggeriert, dass 
er das Filmerlebnis um die Metaebene des Versionenvergleichs - wiederum eine eigene mediale Erfahrung über den reinen kognitiven quellenphilologischen Erkenntnisgewinn hinaus - relational noch erweitern kann.

Man könnte in diesem Sinne in einer etwas weiteren Auslegung die eigentlich metafilmisch gemeinte Bezeichnung der Funktion VIEW FROM Авоve nicht nur als neue Perspektive auf den Film im Modus des Vergleichs von Versionen begreifen. Vielmehr legt der Begriffsgebrauch überdies eine mystifizierende Lesart nahe, die auf eine unsere (Wahrnehmungs-)Welt transzendierende höhere Macht deutet. So erhält die eigentlich quellenphilologisch sich strukturierende Funktion eine fast sakrale Bedeutung: Die Produktion des (buchstäblichen) Glaubens findet beim nutzenden Konsumenten in der performativen Erfahrung der Mise en Relation statt, in der der jeweilige Montagezusammenhang einer Version nur eine augenblickhafte Manifestation zwischen anderen möglichen darstellt. Unter diesen Vorzeichen imprägnieren die Wahl- und Nutzungsmöglichkeiten eines alternativen Filmerlebnisses die aktuelle Version mit Elementen des Transzendenten. Über die diskursive Bezeichnung als Filmklassiker hinaus wird auf diese Weise dispositivisch-räumlich die Konsekrationserfahrung vermittelt, die auf die Relativierung einer Version setzt. Der wählende und vergleichende Zuschauer performt somit kulturhistorische Bedeutsamkeit, wenn er sowohl sich selbst wie auch die Filmversionen zueinander in einer Haltung der Mise en Relation erlebt.

\subsection{Voyeuristische Versprechen des Dispositivs. FifTy Shades of Grey (2015) und Dressed to Kill (1980)}

„As media industries offer consumers the rhetoric of intimacy (i. e., ,secrets" of the cinema) and mastery (i. e., technological expertise of media knowledge), they enhance the sense of owning a personal product".

(Klinger 20o6a: 89)

Eine Variation der Funktionalisierung von vergleichenden Wahrnehmungshaltungen in digitalen Dispositiven stellt die bewusste Verführung zum wiederholten Schauen eines Werkes dar. ${ }^{27}$ Insbesondere im Rahmen des heimischen Raumes kann dies als eine tatsächlich spatiale Form der intimen Aneignung und Bindung an das filmische Werk und dessen Erfahrung verstanden werden.

27 Zum Zusammenhang und zur Bedeutung von Mehrfachschauen und Fankulturen vgl. H. Jenkins (1992: 68 ff.). 
Dabei kann das Mehrfachschauen als Gestus der Einweihung in ein Geheimnis vom Dispositiv evoziert und provoziert werden. ${ }^{28}$ Die folgenden Beispiele machen dies mit ihren spezifischen wirkungsästhetischen Strategien sinnfällig und zeigen dabei zugleich die Inkorporierung der Relativität von Filmwerken in kulturindustriell geprägte, geschichtsbildmodellierende mediale Prozesse.

Letzteres ist bei dem ersten Beispiel insofern interessant, als es sich bewusst um einen relativ jungen Film handelt. Das Exempel zeigt, wie sehr die hier grundsätzlich formulierten Problemstellungen häufig auch bei Editionen aktueller Filme virulent werden, wenn sie in die digitale Zirkulation eintreten. Mittelbar wird darüber deutlich, dass damit die Vorstellung einer zeitlichen Differenz zwischen, älter' und ,aktuell' im Zeichen einer solchen dynamischen Medienumgebung für Filme sich extrem verkürzt und in höchstem Maße relational-kontextuell zu denken ist.

Wichtig ist zudem zu berücksichtigen, was eigentlich der Konsekration und Auratisierung unterliegt: der Film oder das spezifische filmische Erlebnis in der digitalen Reprise durch und mit der DVD oder in einem anderen digitalen Dispositiv? Im Falle der DVD-Edition 2015 (Universal) des sogenannten Skandalfilms Fifty Shades of Grey (Taylor-Johnson, 2015) wird augenscheinlich zunächst auf ähnliche Mechanismen, wie anhand des zuletzt behandelten Beispiels beschrieben, gesetzt, wenn es um den Mehrwert einer DVD in der Offerierung einer weiteren Version geht: Auf der deutschen DVD steht oben auf dem Cover in großer Schrift „Unveröffentlichte Filmversion“, darunter in etwas kleinerer Schrift „Original-Kinofassung“. Was in diesem Beispiel aber als Variante der Konsekrationsakte hinzukommt, ist die Provokation sexuell aufgeladener, voyeuristischer Rezeptionserwartungen, die sich vom Filminhalt auf das Dispositiv und damit auch auf die editierten Versionendifferenzen überträgt. ${ }^{29}$

Der Film erzählt die Geschichte der Beziehung zwischen einer jungen Uniabsolventin und einem Millionär, die sexuelle Grenzen in ihrer Explizitheit vor allem mit BDSM-Praktiken scheinbar überschreitet. Zusammen mit der jungen Protagonistin soll der Zuschauer in diesen neuen sexuellen Lifestyle eingeführt werden. Im Deutschen trägt der Film insofern den ergänzenden Zusatztitel „Geheimes Verlangen“. Auf der Inhaltsebene spricht der Film bereits Schaulust, eine suggestive Rezeptionserwartung an, die auf die fetischisierende

28 Vgl. grundsätzlicher zum Zusammenhang von Mehrfachschauen und dem Markt für das Heimkino Barlow (2005) und Klinger (2006a).

29 Zur generellen „Rhetorik der Intimität“ im Kontext von Heimkinodispositiven und entsprechenden Filmeditionen: Klinger (2006a: 89). 
Ersatzbefriedigung durch den kinematografischen Voyeurismus ausgelegt ist. Die englische Tagline des Films richtet sich noch deutlicher an die Neugier des Zuschauers: „Are you curious?“.30

Im Dispositiv der DVD aus dem Jahre 2015 überträgt sich dies wie folgt auf die Präsentationsform mit Blick auf die Versionenhaftigkeit von Film: Zunächst sieht man sich einem inszenatorisch relativ zurückgenommenen Menü gegenüber. Einzelne Stills aus dem Film sind in einer Collage miteinander kombiniert, die Farben sind den Bildern entzogen und betonen - auf den Titel verweisend - die Grautöne. Im Fokus steht eine größere Aufnahme der beiden Protagonisten, die sich in einem Close-up in einer Profilaufnahme aufeinander fixiert in die Augen schauen (Abb. 7.6).

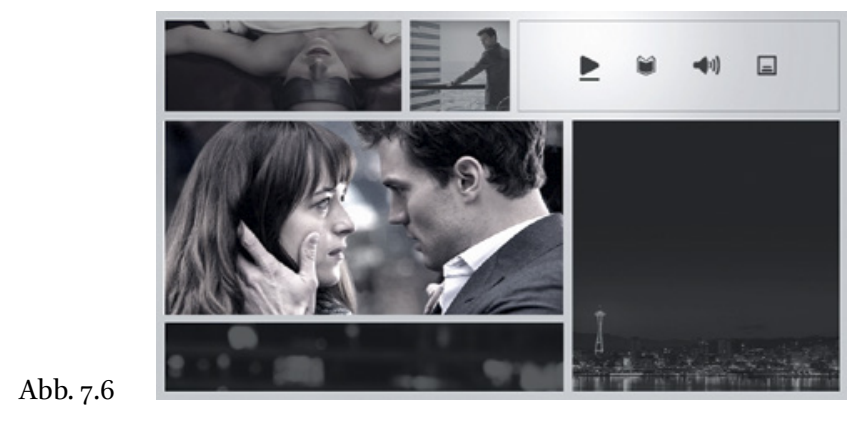

Darüber ist die junge Protagonistin lasziv nackt auf dem Rücken liegend zu sehen, ihre Augen mit einer Maske verbunden. Sie präsentiert ihren Kopf der Kamera. Die simplen Menü-Icons befinden sich rechts oben in Schwarz auf weißem Grund: Play - ein Buch (= Kapitel) - ein Lautsprecher und ein Icon für die Sprachwahl. Was dann aufgrund der simplen Gestaltung der DVD überraschen mag: Wenn man das Play-Icon anwählt, eröffnen sich gleichberechtigt zwei Optionen (Abb. 7.7).

In einer weißen Mittelspalte erscheinen untereinander die beiden anwählbaren Schriftzüge „Kinofassung“, darunter „unveröffentlichte Filmversion“ (wie die DVD-Packung anzeigt: mit alternativem Ende). Die beiden seitlichen rahmenden Bilder aus dem Film erscheinen wie ein Kommentar zu dem, was

30 Vgl. http://www.imdb.com/title/tt2322441/taglines (19.07.2016). Die 2017 erschienene deutsche DVD-Edition des zweiten Teils von FifTy Shades Darker (Foley, 2017) wirbt ihrerseits in Anspielung auf den Maskenball, der in diesem Teil der Trilogie einen tatsächlichen Schauplatz der Handlung darstellt, neben der Originalkinofassung mit einer weiteren „unmaskierten Filmversion“. 


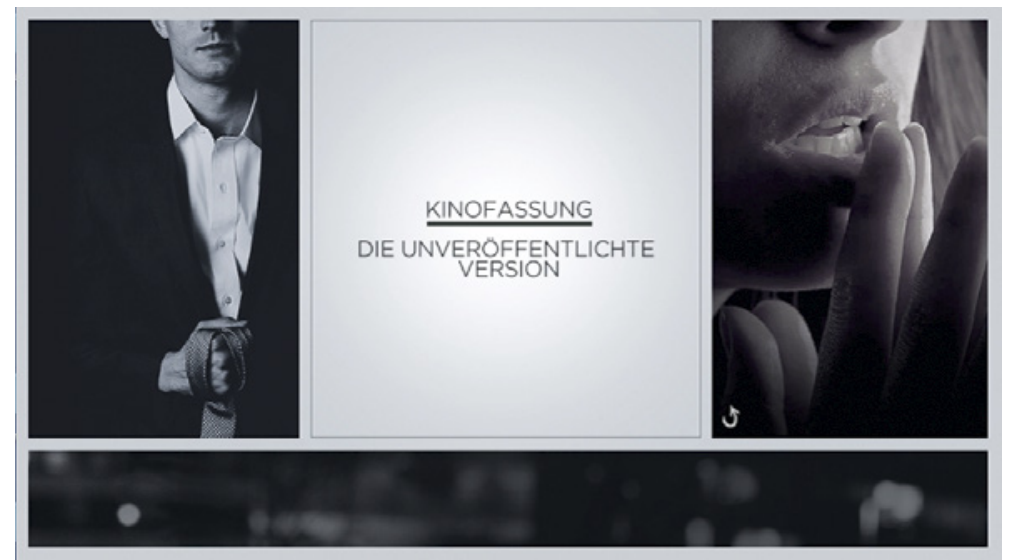

Abb. $7 \cdot 7$

die jeweiligen Versionen versprechen. In der Lese- und damit Schaurichtung von links nach rechts sieht man zunächst auf der linken Seite einen männlichen Torso im Anzug, der um seine Hand eine Krawatte gewickelt hat (eine Andeutung möglicher Fesselspiele). Das wohlgeformte, markante Gesicht des Mannes ist bewusst der begehrlichen Projektion des DVD-Nutzers überlassen, indem es knapp über der Nase abgeschnitten ist und damit die Augen ins HorsCadre des Bildes verlegt sind. Ähnlich mit Andeutungen spielend erscheint das Bild auf der rechten Seite, das ebenfalls in einem grau-schwarzen Farbspektrum in einer seitlichen Nahaufnahme ein weibliches Gesicht zeigt, dessen Mund lustvoll leicht geöffnet ist; Finger streichen sanft über seine Lippen. Der Fokus liegt in vollem Umfang auf dem geheimnisvollen sinnlich-körperlichen Ausdruck, da wieder die Augen außerhalb des Bildes liegen.

Buchstäblich in diesem Rahmen wird man - im Vergleich zu anderen DVD-Menüs - relativ hierarchiefrei vor die Wahl gestellt, welche Version man nun schauen möchte: Die Kinofassung, die als distribuierte Fassung in der öffentlichen Wahrnehmung die maßgebliche Referenz, auch die der öffentlichen Diskussion um den Film, darstellt? Oder aber eine Fassung, die einem als Eigentümer der DVD zu Hause, in dem Aneignungsmodus des Privaten einen exklusiven Einblick in sexuelle Geheimnisse verspricht? Dies bedeutet - eben in Verbindung mit dem Filminhalt - ein zusätzliches voyeuristisches Versprechen auf einen Blick durch das ,Schlüsselloch', hier in Form der DVD als digitalem Dispositiv, dieses vermeintlich neue sexuelle Horizonte zeigenden Films. Das in dieser Art gestaltete Menü verführt von vornherein dazu, den Film in verschiedenen Fassungen mehrfach anzusehen und nicht nur aus der Narration Unterhaltung zu ziehen, sondern aus der 
Erfahrung der aktiven Suche nach Differenz und Wiederholung zwischen den einzelnen Versionen. Hierbei ist interessant festzustellen, dass es - anders als im vorigen Beispiel - keine interaktive Vergleichsoption gibt. Das Menü legt die mediale Erfahrung auf die Sukzession fest. Die Versionen müssen zunächst unabhängig voneinander linear rezipiert werden. ${ }^{31}$ Somit verlegt sich der Vorgang des Vergleichens in die Erinnerung und die Vorstellung des Zuschauers. Dadurch dient die Kopräsenz der Versionen auf einem Träger wieder dem Gefühl der Erweiterung des fiktiven Universums, des tieferen Eintauchens. Die Konsekration vermittelt sich hier im Versprechen der Befriedigung von Schaulust. Es wird der Glaube produziert, man werde in ein sinnliches Geheimnis eingeweiht und erlebe dessen Offenbarung. Damit trägt die DVD zur Auratisierung des fiktiven filmischen Universums bei.

Über die differenzielle Relation der unterschiedlichen Filmversionen wird das diegetische filmische Universum mit einem lustvollen Potenzial weiterer verheißungsvoller $A n$ - und Einsichten aufgeladen.

Gleichzeitig wird mit Blick auf den Zuschauer/DVD-Nutzer und die Funktionalisierung seiner Wahrnehmung eine bedeutsame Verschiebung erkennbar. Im Unterschied zu Re-Editionen, die mit ihrem Begleit- beziehungsweise Bonusmaterial, dem präsentierten Reichtum an filmischen Varianten, letztlich auf den cinephilen Fetisch eines quellenphilologisch formal möglichst vollständigen Werkes setzen, wird bei der hier analysierten Edition von FifTy Shades of Grey das Dispositiv der formalen Zugriffsmöglichkeiten überlagert und bestimmt von der Zurichtung der sexualisierten Schaulust, die sich mit dem spezifischen Sujet des Films verbindet: Voyeurismus und SM-Fesselspiele (in des Wortes doppelter Bedeutung). Dadurch kommt dieser Edition eine Autorität der Sinnlichkeit zu, die die skopophile Neugier und die Wahrnehmung des Zuschauers mit dem Sujet des Films konfiguriert und zum Objekt des Fetischismus selbst werden lässt. Gleichzeitig entscheidet der Zuschauer/DVD-Nutzer über die Selektion im Menu, in welcher Form er der Verführung nach- und sich einer Version der Filmerfahrung hingibt.

Das Versprechen eines Vergleichs im Modus der sexualisierten Schaulust lässt sich mit der Fassungs- wie (digitalen) Editionsgeschichte von Dressed To KILL (De Palma 1980) analytisch weitergehend ausführen, wobei hier aufgrund des größeren zeitlichen Abstands zur Produktionszeit des Films filmhistoriografische Aspekte deutlicher in den Blick geraten. Darüber hinaus

31 Zur Erinnerung: Beim Film gibt es keine Standards und einheitlichen Praktiken, wie sie die aus der Editionsphilologie bekannte Unterscheidung einer historisch-kritischen Edition, einer Studien- und/oder Lesefassung etwa vermittelt; vgl. grundsätzlich zu dem Problem der Re-Edition älterer Filme Bohn (2013b: 297-314). 
speist sich inhaltlich gesehen der pasticheartig angelegte Erotikpsychothriller DRESSED TO KILl narrativ, motivisch und formalästhetisch aus Zitaten und Anspielungen auf bekannte Hitchcock-Klassiker wie REAR WIndow (1954), Vertigo (1958) und Psycho (196o). In einer filmhistoriografisch wirksamen Dimension erfüllt De Palmas Film mit seiner Fassungsgeschichte und seinen digitalen Re-Editionen somit eine doppelte Funktion: Zum einen hat er inhaltlich schon ein postmodernes Verhältnis zur Film- und Kinogeschichte, zum anderen zeitigt seine eigene Editionsgeschichte Zeitschichtungen aus Aktualisierungen und Neukonfigurationen bestimmter Narrative aufgrund von Zensureingriffen. ${ }^{32}$

2001 brachte MGM eine DVD des Films heraus, die mit dem Label Special Edition versehen war. Diese Edition vereinte sowohl die anfänglich öffentlich gezeigte „R-rated theatrical cut“-Version wie auch die explizitere „unrated extended cut"-Version zusammen auf einer Disc (Suarez 2001). ${ }^{33}$ Die ungeschnittene Fassung ist nur 30 Sekunden länger als die zensierte Version und wird von dem Kritiker Suarez wie folgt beschrieben: „What you get with the unrated version is a bit more nudity in the opening shower scene (below the waist), and a few more explicit slashes with the killer's straight razor. Each added shot lasts for a matter of seconds" (Suarez 2001). Lobend hebt Suarez besonders das "full-on special edition treatment" hervor, das MGM auf der DVD 2001 dem Film habe zukommen lassen. Insbesondere gebe es A Film Comparison: The 3 Versions of Dressed to Kill. In diesem Vergleichsclip werden Split-Screen-Konfigurationen gezeigt, in denen Fragmente der verschiedenen Versionen gleichzeitig ablaufen. Sie sollen die verschiedenen inhaltlichen Differenzen zwischen der R-rated, der Unrated und dann (später) der Network-Television-Version des Films illustrieren (Abb. 7.8).

Dieser Zusatz vermittelt als eigener Filmclip nicht nur das philologisch interessante kognitive Wissen um von der Zensur vorgenommene Kürzungen. Vielmehr verspricht der Clip auch gleichzeitig eine Befriedigung der Schaulust auf ein Mehr der unzensierten Fassung - und das bei einem Film, bei dem es inhaltlich ebenfalls um voyeuristische Perversionen und über Blicke ausgelöste Mordphantasien geht. Der Vergleichsclip entwickelt eine ganz eigene Wirkung, da die gezeigte Sequenz, die schon zu Beginn vergleichend aus beiden Versionen (zensiert und unzensiert) simultan abläuft, ganz im Stile seiner filmhistorischen Referenz Psycho (1960), einen lustvoll konnotierten,

$3^{2}$ Zu einem ähnlichen Zensurfall vgl. unten die Analyse von Die Sснӧnste $\rightarrow$ Kap. 7.7.

33 Zur Erklärung der für Editionen wichtigen Speicherkapazität von digitalen Trägern und zu dem Begriff des hier zum Einsatz kommenden Seamless Branching vgl. http://www. bluray-disc.de/lexikon/seamless-branching (19.07.2016). 


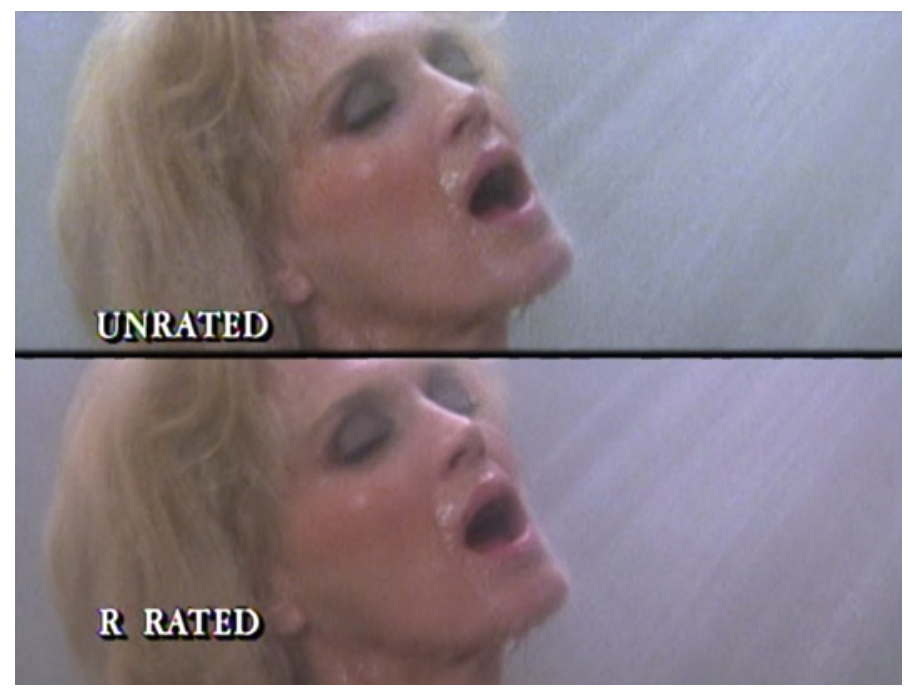

Abb. 7.8

fragmentierten Frauenkörper in Nahaufnahme inszeniert. Der Ausschnitt aus De Palmas Film, der hier aufgenommen wird, spielt mit verschiedenen Blickpositionen und -winkeln. Unterstützt wird die sinnliche Wirkung dadurch, dass diese Split-Screen-Konfiguration mit diegetischem Ton versehen ist zunächst mit einer erotisierenden Melodie, dann in der unzensierten Version bis hin zum Schrei der blonden Frau, die plötzlich unter der Dusche mit dem Rasiermesser angegriffen wird.

Es scheint fast paradox: Stilistisch setzt der eigentlich metafilmische Fassungsvergleich - ,verpackt‘ als DVD-Zusatzbeigabe - fort, was stil- und filmgeschichtlich bei Hitchcock anfing. Hitchcocks berühmte Duschszene fragmentierte über die Montage und die Bildgestaltung den Frauenkörper unter der Dusche, um die Zensur zu umgehen und zugleich den Zuschauer mit seiner Schaulust und sexualisierten Phantasie einzubinden. De Palma greift dies motivisch in postmodern-manieristischer Art und Weise auf (Allen 2014/2011) und stilisiert die angelegten wirkungsästhetischen Elemente. Diese werden nun unter der Maßgabe eines neuen Dispositivs und der damit einhergehenden Warenästhetik, die zunächst einmal quellenphilologisch anmutet, in eine neue Bildkonfiguration - die des Split Screens - überführt. Im Zusammenspiel einer Reprise und der damit einhergehenden Re-Montage generieren die einzelnen wirkungsästhetischen Elemente eine spezifische mediale Erfahrung, die die Fragmentierung des Frauenköpers verdoppelt, wenn nicht gar vervielfacht. Unterstützt durch die Musik ergibt sich so eine neue Wahrnehmungsform des weiblichen Körpers, der auf diese Weise zum multidimensionalen Lustobjekt wird und der multiplizierten Schaulust des 
Zuschauers anheimgegeben ist. Dies findet sich noch unterstützt durch bildgestalterische Elemente, die bereits im Film enthalten sind, wie etwa in Form von Spiegelungen oder Aufnahmen durch den Wasserschleier des Duschstrahls. Insofern ist Suarez' Fazit zu der DVD-Edition 2001 sehr wörtlich zu nehmen:

Still, long time fans of this film will be more than pleased with MGM's work with this DVD. The audio and video presentation of this disc are exceptional given the film's age $[. .$.$] and the quality of the supplements make the disc even more$ attractive (Suarez 2001, Hervorh. FH).

Was hier deutlich wird: Der Fokus der Auratisierung hat sich verschoben. Es ist nicht nur das historische Filmwerk Dressed to KiLL, das Objekt der Begierde wird. Vielmehr ist es nun auch die (digitale) Überlieferungsgeschichte des Werks als Cluster, die neue mediale Phänomene ausbildet, welche ihrerseits Sehsüchte kreieren. Was eigentlich als warenästhetisches Mehrwertversprechen des Trägers und der Zugangsform gedacht war, trägt zur Fetischisierung bestimmter exklusiver ästhetischer Aspekte des Films bei und überträgt diese - ohne die in De Palmas Film angelegten kritischen selbst-reflexiven Brechungen - in eine neue mediale Konfiguration: Der Film, spezifische Wahrnehmungsstrategien, seine Überlieferungsgeschichte und Zugangsform gehen wirkungsästhetisch eine Symbiose ein, wobei gerade in der Vervielfachung der Perspektiven die wechselseitige Auratisierung liegt. Hervorzuheben an dem Fall ist darüber hinaus, dass sich diese Form der clusterförmigen Auratisierung in digitalen Distributionskanälen in der Zeit fortschreibt - was wiederum neue ästhetische Formen der Verhältnissetzung kreiert, die ich in ihren Effekten als die Konsekrationserfahrung begreife.

2015 hat Criterion dann eine Blu-Ray-Special-Edition des Films veröffentlicht, in deren Rahmen der oben beschriebene Clip über die Zensur des Films aufgegriffen und re-kontextualisiert wurde. ${ }^{34}$ Mit Bezug auf diese Edition von Criterion wird von der Rezensionswebseite DVDBeaver.com besonders betont, was auch das entsprechende Begleitheft der Blu-Ray-Disc selbst in Hinblick auf Autorschaft hervorhebt: „Transfer supervisor: Brian De Palma; Colorist: Lee Kline/Criterion, New York“ (Tooze 2011-2015). An dieser Darstellung fällt auf, dass De Palmas Autorschaft ${ }^{35}$ sich in diesem Diskurs nicht mehr nur auf die Inszenierung des Films selbst, sondern auch auf den digitalen Reproduktions-

34 Die Edition beinhaltet - so die Beschreibung von Criterion selbst - „[p]ieces from 2001 about the different versions of the film and the cuts made to avoid an X rating“; vgl. die Criterion-Website https://www.criterion.com/films/28686-dressed-to-kill (19.07.2016).

Vgl. zum weiteren Diskurs um DVDs und auteurism Grant (2009). 
prozess des Films - den Transfer in die digitale Domäne - bezieht. Durch die weiteren genauen Angaben der verwendeten Ausgangsmaterialien und der Aufnahmeapparatur (Scanner etc.) vermittelt Criterion eine aus quellenphilologischer Perspektive zu lobende Transparenz im Vergleich zu sonst meist nur bedingt informativen Angaben in der digitalen Filmdistribution. ${ }^{36}$ Gleichzeitig geraten diese Informationen zu warenästhetischen Elementen der Edition des Films und tragen zu dessen Konsekration bei.

Das Prestige - die kulturelle Autorität - wird durch das Versprechen der vom Autor bezeugten historischen Authentizität kreiert. Gleichzeitig werden alle Entscheidungen - so die öffentlich sichtbare Darstellung von Criterion - dem Ziel untergeordnet, ein möglichst ungebrochenes immersives ästhetisches Erlebnis des Filminhalts zu ermöglichen. ${ }^{37}$ So wird auch der Verantwortliche für die digitale Licht- und Farbbestimmung (Color Grading) zum nobilitierenden Koautor, da er die Farbanpassung unterschiedlicher, hier zusammengeführter Versionen vorgenommen hat - zugunsten einer möglichst in sich kohärenten ästhetischen Erscheinung des Films. ${ }^{38}$ Das ausgewählte filmrestauratorische

$36 \quad$ Der Kritiker Tooze (2011-2015) zitiert das Begleitheft von Criterion noch ausführlicher, in dem wie folgt geschrieben wird: „The version of the film included here is director Brian De Palma's original and preferred unrated cut. Supervised by De Palma, this new digital transfer was created in $4 \mathrm{~K}$ resolution on a Scanity film scanner from the $35 \mathrm{~mm}$ original camera negative; a $35 \mathrm{~mm}$ interpositive was also used for additional footage for this cut. [...] Thousands of instances of dirt, debris, scratches, splices, and warps were manually removed using MTI's DRS, while Digital Vision's Phoenix was used for small dirt, grain, noise management, jitter, and flicker" (Hervorh. FH). Hier wird in vielerlei Hinsicht deutlich, wie bei aller Dokumentation der Quellenlage, der verwendeten Technologien und damit des Digitalisierungsdispositivs auch in den Formulierungen Konsekrationsrhetorik zum Tragen kommt: etwa die unter anderem an der Person Brian De Palmas festgemachte Re-Autorisierung des Digitalisierungsprozesses, die Vermittlung des großen Aufwands des Restaurierungsprozesses u. v. m.

37 Dies geschah vor allem durch das Anpassen der Farben, sodass die unterschiedlichen filmischen Quellenmaterialien nicht mehr im Fluss des Films zu unterscheiden sind. Insofern sind auch Gebrauchsspuren (Fehler und Schäden) entfernt worden.

38 Vgl. ähnliche Praktiken in Hinblick auf die Nobilitierung einzelner analoger Filmkopien. Solche Praktiken der personenorientierten Konsekration sind im Kontext der Deterritorialisierung von Filmen im digitalen Zeitalter zu sehen: So wurde etwa auf dem Festival Il Cinema Ritrovato in Bologna im Jahr 2016 nicht nur die Aufführung des Films The BAnd Wagon als Starmusical mit Fred Astaire und als ein Zeugnis des Technicolor-Farbverfahrens anlässlich eines Screenings auf der prestigeträchtigen Open-Air-Vorführstätte der Piazza Maggiore präsentiert; zudem wurde vor allem die Besonderheit prominent ausgestellt, dass die projizierte Vorführkopie aus der Sammlung von Martin Scorsese stammte. Insofern fungierte - ganz im Sinne Bourdieus (2011c) der Sammler beziehungsweise der Besitzer des Kunstobjekts in diesem Kontext als wertsteigernde Größe zum Zweck der Bildung von symbolischem Kapital. 
Wissen, das sich hier mitteilt, ist als Teil von Konsekrationsakten im Zeichen von Digitalisierungsprozessen und digitalen Editionen zu verstehen.

Dies ist wiederum im Austauschverhältnis mit dem Filmerlebnis zu sehen. In $\rightarrow$ Kap. 5 habe ich bereits im Kontext von Restoration Talks zu digitalen State-of-the-Art-Technologien entwickelt, wie der Zuschauer über den lustvoll-immersiven Konsum des digitalisierten Films ebensolche Formen der Konsekration (nach)vollziehend affirmiert. Ich habe diese Form der wirkungsästhetischen Vergegenwärtigung des Technoimaginären digitaler Restaurierungen, welche sich über das Erleben des restaurierten Filmes vermittelt, auch digitale Performance genannt ( $\rightarrow$ Kap. 5 ).

In der digitalen Überlieferungsgeschichte von DRESSED TO KILL zeigen sich darüber hinaus noch weitere Phänomene, die den Zuschauer/Nutzer als (Nach-)Vollzugsinstanz in Vermittlungen digitaler Qualitäten des Films einbinden - wieder vorrangig über Modi des Vergleichens: Auf DVDBeaver.com vergleicht Tooze 2015 nicht mehr nur die (analogen) Filmversionen, sondern der Fokus verschiebt sich auf die digitalen Versionen, die sich wiederum im Zeichen ihrer Dispositive in die Fassungs- und Versionengeschichte einschreiben. Hierbei werden neue Bildregister kreiert. Zum einen sind dies vergleichende Konfigurationen von Graphen und Bitraten, die in abstrakttechnizistischer Form illustrieren sollen, wie viel Information, welche Datenmengen sich auf der DVD als Film befinden und wie sich diese in der Zeit verhalten und verteilen (Abb. 7.9). ${ }^{39}$

39 Hier sei erneut darauf hingewiesen, dass etwa die ästhetischen Ausprägungen von ,Metatexten` zu Filmen auf Webseiten fruchtbar mit Debatten um die Produktivität einer cinephilen, partizipatorischen Fankultur (u. a. H. Jenkins 1992) in der Digitalkultur weiterzudenken wären. Eine vertiefende Einlassung würde aber an dieser Stelle den Argumentationsrahmen in Hinblick auf meine spezifische Fragestellung sprengen; vgl. einführend zu dem Zusammenhang von digitaler Fankultur, Produktion, Ökonomie, Kulturindustrie und Konsum überblicksartig in einer ethnografisch imprägnierten Perspektive Einwächter (2014: bes. einführend 9 ff.). Den Schlüsselbegriff stellt hier - insbesondere in einer digitalen Medienumgebung - der Neologismus Produser beziehungsweise Produsage dar (Bruns 2008). Zu den Aspekten solcher Erscheinungen in Bezug auf Fragen der Erinnerungskultur im Kontext des Internets als „Archive des Affekts“ vgl. Gehl (2011), zit. n. Einwächter (2014: 190).

$\mathrm{Zu}$ den abstrakten Bildregistern der im vorliegenden Gegenstandsbereich entstehenden Darstellungsmodi von, digitalen Eigenschaften - etwa in Form von Graphen wären erweiternde Anschlüsse an Überlegungen der Diagrammatik denkbar: So hat etwa Krämer (2009) über die visuelle Epistemologie der abstrakten Darstellungsformen nachgedacht. Flückiger (2011) reflektiert Rodowick (2007), wenn sie die Präsentation von Problemstellungen in Diagrammen angesichts filmischer Phänomene als „metaphorische Vermittlungsinstanz" zwischen Bildlogik und Sprachlogik beschreibt: als einen „Schritt 


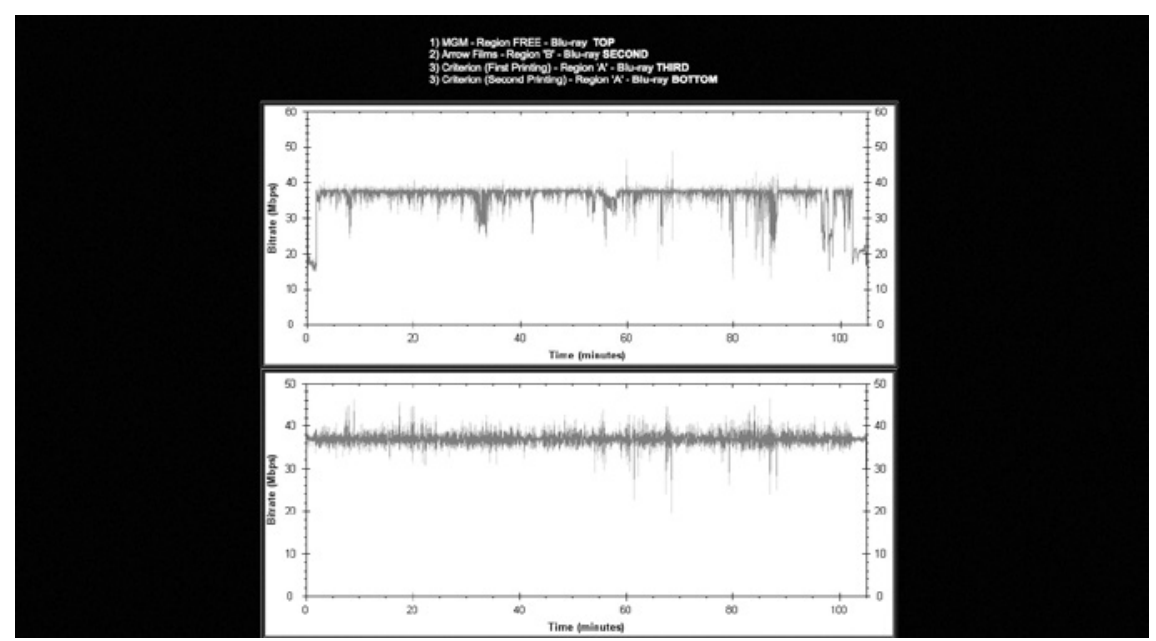

Abb. $7 \cdot 9$

Auf der wirkungsästhetischen Ebene werden somit neuartige Bildvergleiche kreiert, die nur noch im pragmatischen Kontext mit dem Film als Digitalisat zusammengebracht werden können. Aber auch hier wird in der Abstraktion ein vergleichender Blick etabliert, der eine Aussage über die Qualität des Films als Digitalisat vermitteln soll. Damit schreibt sich dieses technizistisch argumentierende Bildregister in den warenästhetischen Diskurs um die heutige Zugangsform des Films ein.

Auf der anderen Seite werden auf (solchen) Foren und Rezensionswebseiten neue metafilmische Bildserien produziert, die sich im Gegensatz zu der abstrakten Visualisierung allerdings noch im mimetischen Bereich bewegen. Es werden konkrete Bildvergleiche verschiedener Filmeditionen konfiguriert (Tooze 2015). Hier werden einzelne Stills des Films aus den jeweiligen Versionen und Editionen neben- oder untereinander dem Benutzer der Webseite zum Nachvollzug des Vergleichs offeriert (Abb. 7.10).

zwischen konkreter Anschaulichkeit hin zur konzeptuellen Abstraktion“ (Flückiger 2011: 59-6o). Vgl. zu Visualisierungsstrategien von filmischen Strukturen im Kontext filmwissenschaftlich ausgerichteter Digital Humanities Heftberger (2016). In dem hier diskutierten Zusammenhang werden die Bildserien im kulturindustriellen Kontext allerdings vornehmlich als Teil der Warenästhetik und damit Konsekrationserfahrung in wahrnehmungstheoretischer Perspektive gesehen. 


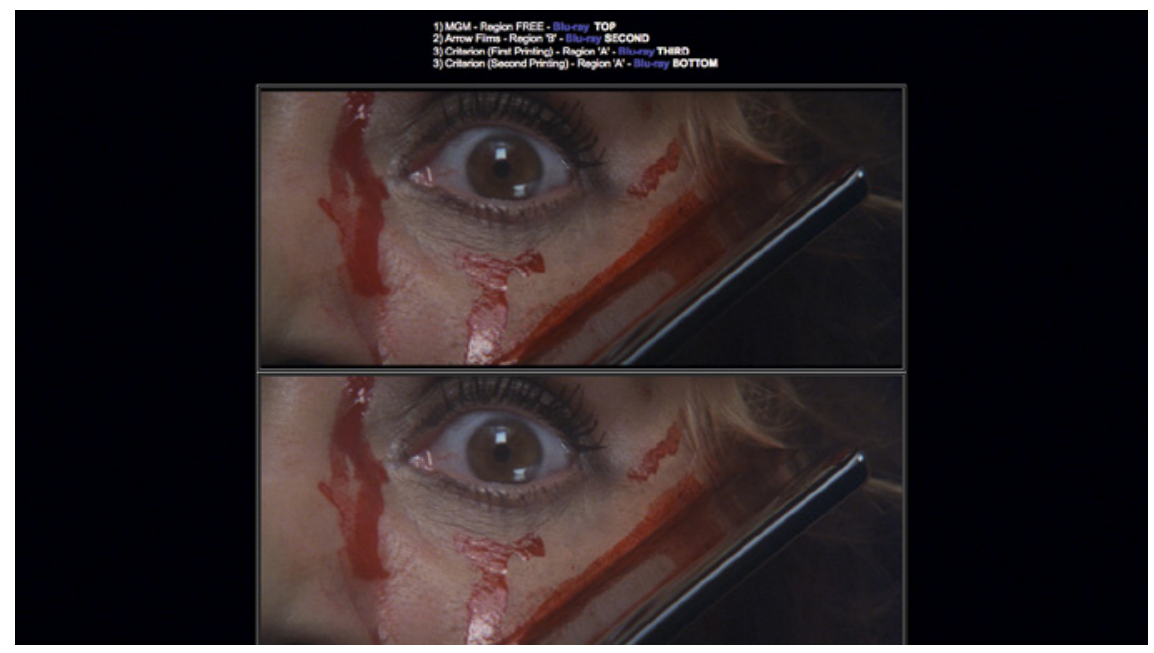

Abb. 7.10

Es ist eine spezifische Art und Weise der Evidenzproduktion, die die Bilder buchstäblich einer warenästhetischen Revision unterzieht, indem sie stillgestellt, neu und selbstreferenziell - aus ihrem narrativen Kontext gerissen montiert werden. Die Projektion des Lesers erfüllt in dieser Konfiguration eine wichtige Funktion. Es geht darum, den Fortschritt der Technik des digitalen Transfers tatsächlich in den Bildvergleichen zu suchen und wiederzufinden. Hier überlagern sich verschiedene Diskursebenen. Zum einen sind es Diskursformationen, die sich auf den faktischen Digitalisierungsprozess (Qualität des Transfers), auf das Speichermedium oder das Wiedergabegerät ${ }^{40}$ beziehen. Zum anderen sind auch pragmatische Aspekte der Diskursbildung im seinerseits digitalen Medium des Internets mit seinen Darstellungs- und speziellen Kommunikationsformen von Bedeutung.

Was bleibt nun festzuhalten über den Film Dressed to Kill heute? Die Fassungsgeschichte, die mit der kulturellen Praxis der moralischen Normierung - der Zensur - begann, entwickelte zunächst im Kontext digitaler Dispositive als Distributionsmedien einen Paratext mit eigener Ästhetik, die die im Film bereits angelegte postmodern-manieristisch angelegte Schaulust in ein neues Format überführt - allerdings eher unfreiwillig. Die in De Palmas Film erkennbaren medienkritischen Brechungen der Bilder gehen in der Re-Konfiguration des Vergleichsclips weitgehend verloren. Mit weiteren digitalen Versionen des Films gehört dieser ästhetisch vermittelte Versionenvergleich allerdings nun zum Dispositiv und unterliegt selbst einer Reprise.

40 DVDBeaver.com ist hier bemerkenswert transparent und gibt darüber Auskunft. 
Darüber hinaus gesellt sich mit den nach 2001 folgenden Versionen ein weiterer Aspekt der Warenästhetik des Films unter den Vorgaben des technizistischen Diskurses im Internet um digitale Qualität hinzu. Dabei entstehen neue Bildregister und -serien, die wiederum in der Öffentlichkeit des Internets einer ständigen Mise en Relation im Dienste einer Evidenzkonstruktion über das Verhältnis der Versionen zueinander unterliegen. Es entfalten sich hier und auch über andere intermediale Paratexte immer wieder neue Zuschreibungen an Instanzen einer Urheber- beziehungsweise Autorschaft, welche für die diskursive Konsekration und Auratisierung des Films sowie der jeweiligen Edition funktionalisiert werden - dies gilt auch für die Re-Autorisierung des digitalen Transfers und der entsprechenden Bearbeitung. Gleichzeitig wird etwa auf Webseiten versucht, über metafilmische Bildregister in der mathematischen Abstraktion von Diagrammen eine desanthropomorphisierte, objektivierende Instanz zu etablieren, die über technizistische Evidenzkonstruktionen die Bedeutungsautorität zur digitalen Qualität des Films vermitteln soll. ${ }^{41}$

Der Rezipient steht in all diesen Konfigurationen als affirmierende (Nach-) Vollzugsinstanz des dispositivisch und ästhetisch inszenierten Vergleichs im Zentrum der nachhaltigen Wirkung des medienhistorischen und filmischen Clusters Dressed to Kill, bei dem es zunehmend schwierig wird, einen stabilen (historischen) Ausgangspunkt festzulegen.

Letzterer Befund ist bewusst allgemein formuliert, weil es hier nicht allein um das Problem der Bestimmung eines eindeutigen, historisch fixierbaren und verortbaren filmischen Originals gehen kann.

Vielmehr legen meine Überlegungen nahe, das Augenmerk auf die wirkungsästhetische Dimension zu richten, in deren Rahmen es zu einer nachhaltigen, clusterhaften Verwebung des fiktiven Universums des Filminhalts mit werkspezifischen ästhetischen Elementen des Films sowie der Warenästhetik der jeweiligen Form der digitalen Reprise kommt. Dies zeitigt Folgen für die Wahrnehmung des Films sowie seiner Geschichte(n).

Wenn De Palma in seinem Film über Prinzipien des Pastiches und des Zitats einen neuen, re-konfigurierenden Blick auf Hitchcock und damit auf die Geschichte der filmischen Schaulust ermöglichen will, so liest sich vor diesem Hintergrund Toozes Fazit zu seiner Editionskritik als Fortschreibung dessen

41 Zur Bedeutung einer partizipatorischen Fankultur in diesem Zusammenhang vgl. die ausführlichen Anmerkungen oben etwa zu H. Jenkins (1992) und Einwächter (2014). $\mathrm{Zu}$ abstrahierenden Bildregistern im Feld der Diagrammatik vgl. Krämer (2009) und Flückiger (2011). Zu weiteren Visualisierungsstrategien im filmwissenschaftlichen Kontext und einer an den Formalismus angelehnten Filmanalyse vgl. Heftberger (2016). 
auf der Metaebene - und damit als ein überraschendes Zeugnis der Verschmelzung von filmischer Fiktion und digitaler Überlieferung: Die Blu-RayDisc-Criterion-Ausgabe von 2015 sei aufgrund ihrer Gestaltung und Austattung zu empfehlen "to those fans looking for a new angle of their viewing“ (Tooze 2015, Hervorh. FH).

Hier gilt insofern: Eine bereits in sich pasticheartige filmästhetische Auseinandersetzung mit der Geschichte kinematografischer Skopophilie und voyeuristischer Bedürfnisbefriedigung bildet in ihrer Überlieferung in der digitalen Domäne auf der Ebene des Dispositivs weitere Elemente aus, die den Filmzuschauer und Dispositivnutzer dazu verführen, zur (Nach-)Vollzugsinstanz medienhistorischer Referenzen und kultureller Bedeutsamkeit zu werden - und dies dezidiert im Modus des Sinnlich-Begehrenden: der Lust an der Mise en Relation.

\section{6 „That is how it could have happened ... but how about this“. Möglichkeitsdimensionen eines Filmwerks. Clue (1985), Memento (2000) und BLAde RunNer (1982)}

Nachstehend werden einige Fälle besprochen, die aufgrund ihrer variantenreichen Fassungs-, Überlieferungs- und Distributionsgeschichte erst in bestimmten Dispositiven und Zugangsformen ihre spezifische Wirkung entfaltet und damit ihr heutiges Prestige erlangt haben. ${ }^{42}$ Gemeinsam ist den Beispielen, dass die von ihnen erzählte Geschichte sich jeweils im Kontext variierender Formen der Mise en Relation verändert. Diese Versatilität der filmischen Erzählung gerät in der Folge zum Mehrwert im DVD-/Blu-Ray-Dispositiv. ${ }^{43}$ So realisiert sich etwa im offensiv eingeforderten seriellen Mehrfachschauen, im aktiven Aufsuchen von Differenzen und Wiederholungen seitens des $\mathrm{Zu}$ schauers der auratisierende Mehrwert der Filmwerke, die - so meine These in medialen Clustern aufgehen. Es geht in den nachstehenden Überlegungen insofern darum, die Konsequenzen für das Erlebnis des Films (beziehungsweise Filmwerks) fassbar zu machen. Dabei sei an dieser Stelle erneut mein Ansatz hervorgehoben, die Verschmelzung narrativer und filmästhetischer Merkmale des digitalisierten Films mit der jeweiligen Versionengeschichte und deren Aktualisierung in digitalen Dispositiven in den Fokus zu nehmen und als medienhistoriografisch erfahrungsbildend zu verstehen.

42 Für den Hinweis auf das Beispiel Clue danke ich Celia Geering.

43 Zum Problem des Zugangs über Internet, Streaming und VoD vgl. weiter unten. 
Im Folgenden werden nun Filme in ihrer digitalen Reprise untersucht, die mit Blick auf ihre Erzählhaltung und narrative Struktur von vornherein mit alternativen Möglichkeitsdimensionen spielen und damit grundsätzlich ein eindeutiges, verlässlich dechiffrierbares zeichenhaftes Verhältnis zur Welt infrage stellen. Es sind Filme, die auf ihrer Inhaltsebene alternative Plotentwicklungen durchspielen. Eines der bekanntesten und wohl elaboriertesten Beispiele hierfür ist Alain Resnais' Zwillingsfilm SMoking/No SMOKIng (Resnais, 1993), ${ }^{44}$ dessen narrativer Aufbau sich nicht linear entwickelt, sondern - abhängig von der Entscheidung, ob die Protagonistin sich am Anfang eine Zigarette anzündet oder nicht - sich wie ein Entscheidungsdiagramm entfaltet. Anschaulich ist diese Struktur in grafischer Form im Booklet der DVD-Edition (Pathé Video, 2004) erklärend aufbereitet (Abb. 7.11).

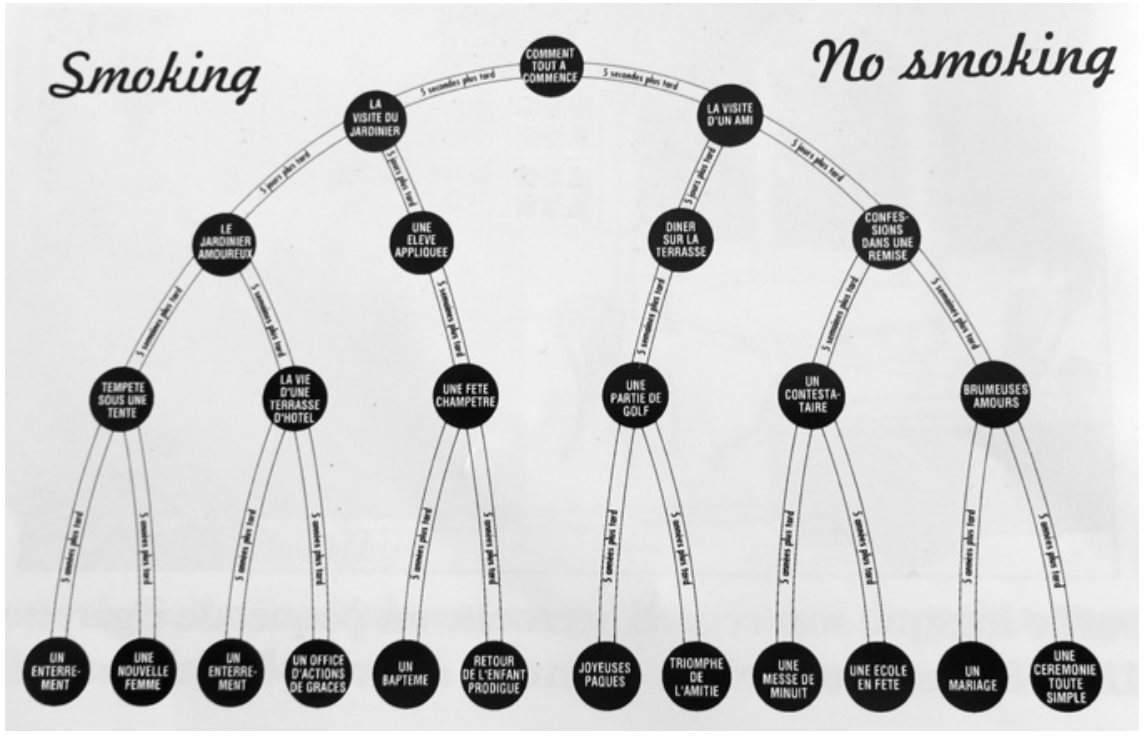

Abb. 7.11

Im Verlauf von Resnais' Film wird die Handlung mit ziemlicher Regelmäßigkeit in Entscheidungssituationen angehalten, wird der Erzählfluss durch Zwischentitel - wie in derStummfilmpraxis - unterbrochen und die Option auf

44 Zum ästhetischen Vorbild der sogenannten „potenziellen Literatur“, deren strukturellen Merkmalen und Funktionsweisen vgl. auch H.-B. Heller (2001b). Dies schließt auch an meine Ausführungen zu (Erzähl-)Formen des Offenen als Modi der Einbindung des Rezipienten über Erinnerungsaktivitäten an, vgl. Scherer (2001). 
potenzielle Alternativen der Handungsfortführung markiert („ou bien“/,oder auch“) - um sich dann zunächst für eine bestimmte Variante zu entscheiden.

Resnais' Film wird - unter anderen - von David Bordwell in dem Aufsatz „Film Futures“ (2007/2002: 185) zitiert, in welchem der Filmwissenschaftler mit Rekurs auf erzählerische Strukturexperimente des Schriftstellers Jorge Luis Borges das Modell eines sich labyrinthisch entfaltenden forking path für den Film entwickelt. Vor dem Hintergrund der vielfältigen Rezeption von Bordwells Überlegungen in der Filmnarratologie fasst Britta Hartmann 2012 die Merkmale der entsprechenden filmischen Erzählungen wie folgt zusammen:

Geschichten mit alternativen Plotentwicklungen, die nacheinander durchgespielt werden. [...] Gemeinsam ist diesen Filmen, dass hier von verschiedenen möglichen Entwicklungen der gleichen dramatischen Grundkonstellation erzählt wird, die von einem gegebenen Zeitpunkt und einem alltäglichen Ereignis ihren Ausgang nehmen und zufalls- oder schicksalsgesteuert sind. Doch wo es keine ,richtigen', sondern lediglich alternative, bestenfalls ,wünschenswerte Plotentwicklungen gibt, kann es auch keine ,falschen' geben. Der Wahrheitsund Geltungsanspruch der Geschichten ist ausgesetzt, wird möglicherweise überlagert von der subjektiven Beurteilung des Geschehens (Hartmann 2012).

Was bei Bordwell schon angelegt ist (2007 und 2006), findet unter anderem bei Buckland (2009) und vor allem bei Elsaesser (2009) in Verbindung mit Begriffen wie multiple-draft-narratives, puzzle film und mind-game-film (auch übersetzt mit "Gedankenspielfilm“, Schmerheim 2014) weitere Ausformulierungen: Diese gestalterischen Ausprägungen werden in direktem Zusammenhang mit digitalen Medienumgebungen und den dadurch geförderten Kompetenzen der Rezipienten/Nutzer gesehen. Schon Bordwell verweist auf Computer- und Videospiele (2007: 187) und deren Einfluss auf erzählerische Logiken mit verschiedenen Handlungsoptionen und -levels. Daran anknüpfend greift Klinger (2006a: 157) den Begriff puzzle film auf, um strukturelle Eigenschaften auszumachen, die besonders im Heimkinobereich zum mehrfachen Ansehen eines Films anregen (u. a. auch Schmerheim 2014) und die sich dadurch besonders in die persönliche Erinnerung des Rezipienten einschreiben.

Damit wird deutlich: Neben der Einschätzung etwa einer Forking-pathStruktur als inhaltliche Kategorie in Form eines Entscheidungsmoments innerhalb der Narration (ob vom Zufall oder Schicksal gesteuert) werden explizit solche Ausprägungen des Erzählens im Kontext digitaler Medientechnologien und ihrer Möglichkeiten der entlinearisierten, vernetzten Hyperlinkverknüpfungen gesehen. Der auch für elektronische Literatur gebrauchte Begriff des Hypertextes beziehungsweise der Hyperfiktion (Mahne 2007 und 2006; Suter 2000), welche sich eben nicht mehr linear, sondern interaktiv rezipierbar über verräumlichte Linkstrukturen entfaltet, wurde in diesem $\mathrm{Zu}$ sammenhang von Espen Aarseth (1997) als Variante von ergodischem Erzählen 
bezeichnet; dieses setze einen aktiveren Rezipienten voraus, welcher sich auf die spielerischen Formmuster, die die Wahlmöglichkeiten eröffneten, einlässt.

Wie zu Beginn dieses $\rightarrow$ Kap. 7 bereits ausgeführt, kommt auf der technischepistemischen Voraussetzungsebene der digitalen Domäne an dieser Stelle besonders jenes Prinzip nach Flusser (1992) zum Tragen, das auf der Möglichkeit des Random Access digitaler Daten basiert. Vor allem Distelmeyer (2012) leistet den Transfer dieses Diskurses auf das Prinzip der zu navigierenden Verräumlichung auf die konkreten digitalen Erscheinungsbedingungen von Film in entsprechenden Dispositiven wie Blu-Ray-Disc oder DVD (Distelmeyer 2012: $17 \mathrm{O} \mathrm{ff}$.).

Im Folgenden untersuche ich solche dispositivisch wirksamen Netzwerkstrukturen, welche bestimmte Ausprägungen der sinnkonfigurierenden Mise en Relation eröffnen und die sich wiederum mit dem Inhalt sowie dem formalen Aufbau des editierten Films verweben, der seinerseits mit Forkingpath-Mustern arbeitet. Zusammen, verstanden als Cluster, tragen solche Netzwerkstrukturen dergestalt bei zur Konsekration im Erfahrungsmodus der Relativierung.

Das zunächst diskutierte Beispiel beginnt mit seiner Fassungs- und Versionengeschichte schon im vordigitalen Zeitalter, was es für meine Fragestellung als historisches Exemplum besonders interessant macht. Als der Film CluE unter der Regie von Jonathan Lynn 1985 als Verfilmung des gleichnamigen bekannten Detektivbrettspiels in die Kinos kam, waren - um den Möglichkeitsdimensionen des Spiels Rechnung zu tragen - bereits ursprünglich vier verschiedene Enden der Whodunit-Story gedreht worden; vier unterschiedliche Versionen des Schlusses, in denen jeweils eine andere Figur der Mörder war. Allerdings wurde das vierte Ende bereits während der Schnittphase verworfen, insofern kamen nur drei verschiedene Schlüsse in Umlauf, was 1985 unter anderem aus ökonomischen Erwägungen heraus in eine spezifische Distributions- und Präsentationspolitik umgesetzt wurde: „Three different endings, all of which plausibly work, were ultimately filmed. Theaters were then sent a copy of the movie containing either ending A, B or C. In theory, it would have allowed superfans to go see the film multiple times" (Rawden 2015). Aber dieses Distributionsmodell scheiterte, offensichtlich waren die $\mathrm{Zu}$ schauer nicht bereit, mehrfach den Kinoeintritt für unterschiedliche Enden zu bezahlen (Rawden 2015). Laut eigenen Aussagen des Regisseurs Jonathan Lynn wurde dann auf sein Betreiben hin schon für die erste Veröffentlichung auf dem Home-Video-Markt auf VHS eine neue Version erstellt, ${ }^{45}$ die Rawden 2015 rückblickend wie folgt beschreibt - und womit er gleichzeitig die Überlieferungslinie von VHS zu den digitalen Trägern zieht: „[W]hen [CLUE] was 
finally released on VHS, DVD and Blu-Ray, the endings were compiled together and shown one after another rapid fire style" (Rawden 2015).

Zum genaueren Verständnis: Bis zu einem gewissen Punkt ist die Geschichte, das Mordrätsel in allen Versionen identisch. Eine Gruppe verschiedener Figuren trifft sich in einem genrestereotypen Haus im Tudorstil auf eine mysteriöse Einladung hin zu einem Dinner: Mrs. Peacock (Eileen Brennan), Mrs. White (Madeline Kahn), Professor Plum (Christopher Lloyd), Mr. Green (Michael McKean), Miss Scarlett (Leslie Ann Warren) und Colonel Mustard (Martin Mull). Arrangeur der Situation scheint zunächst der Butler Wadsworth (Tim Curry) zu sein, der als Erster auf dem Anwesen eintrifft. Unterstützt wird er anfangs von dem übersexualisierten Hausmädchen Yvette (Colleen Camp). Keiner der Gäste weiß, warum sie zu diesem Treffen mit einem anonymen Brief eingeladen wurden. Sie sind angehalten, ihre wahren Identitäten und Lebensgeschichten nicht zu offenbaren. Die oben genannten Namen sind auferlegte Pseudonyme (die den Spielfarben der Figuren im Brettspiel entsprechen). Als Mr. Boddy (Lee Ving) schließlich zu der Gesellschaft stößt und sich als einzige Bezugsperson aller Protagonisten entpuppt, wird er sogleich in einem Moment der Dunkelheit - das Licht geht tatsächlich aus - zur ersten Leiche („body“). Danach entwickelt sich aus den Vermutungen über Hintergrundgeschichten, Täterschaft, Verhältnisse und Motive ein rasantes Spiel mit kognitiver wie räumlicher Verwirrung: Der Aufbau des Hauses ist angelegt wie das Tableau eines Brettspiels, in dem die verschiedenen Räume die Spielzüge zueinander definieren. Es kommt zu temporeichen Raumwechseln jeder einzelnen Figur, wobei immer wieder die zwischenmenschlichen wie auch die tatsächlichen räumlichen Beziehungen neu definiert werden. Konkretisiert wird dies etwa in den Geheimpassagen als Verbindungen zwischen eigentlich getrennten und nicht zugänglichen Räumen.

Dieser spielerische Umgang in der Erzählweise wird unterstützt durch ein äußerst theatral-expressives und körperbetontes, auf Slapstick angelegtes Schauspiel der Darsteller, die das Spiel mit dem Schein zur Schau stellen. Dies wird ergänzt durch den omnipräsenten Einsatz von doppeldeutigen Sprachspielen - bis hin zur mehrdeutigen Selbst-Reflexion des dramaturgischen Aufbaus als Whodunit, wenn wiederholt der Begriff Red Herring zitiert wird. Der Begriff ist bekanntlich im Kontext von Spannungsdramaturgien über Äußerungen von Alfred Hitchcock populär geworden (Truffaut 2010/1966: 263). Er bezeichnet das Verfahren, den Zuschauer über gezielte Informations-, Aufmerksamkeits- und Sympathielenkung an eine Figur oder an eine Situation emotional zu gewöhnen, um dann auf der Basis dieser Gewöhnung einen Umschlag oder Entzug von Sicherheit erfolgen zu lassen - zugunsten einer erhöhten Schockwirkung und Irritation. Das wohl bekannteste 
Beispiel einer Red-Herring-Dramaturgie ist das gewaltsame Verschwinden von Marion Crane aus der Erzählung von Psycho (Hitchcock, 1960) durch ihre Ermordung unter der Dusche. Ihr Tod kommt umso überraschender und schockierender, als Marion zuvor als Protagonistin und Identifikationsfigur inszeniert wurde. ${ }^{46}$

Am Ende von Clue behauptet der Butler Wadsworth, er wisse, wer die ganzen Morde im Haus begangen habe. In einer rasanten Jagd durch die verschiedenen Räume der Handlung wiederholt Wadsworth im Modus des Re-Enactment die Taten, bis er schließlich mit Ankündigung den Stromausfall erneut herbeiführt und damit ostentativ-theatral - wie auch schon beim ersten Mord - das Filmbild in Dunkelheit taucht. Damit wird der Zuschauer zum spekulierenden Mitspieler gemacht, indem die Sichtbarkeit des Schauspiels mit Leerstellen durchsetzt wird. Auf diese Weise liefert der Butler zudem metaphorisch die narrative Ausgangssituation, nun Licht ins Dunkel des Rätsels bringen zu können. Ab hier offeriert die DVD/Blu-Ray-Disc - auch im Unterschied zur VHS - nun diverse Optionen. Bereits vor der Filmsichtung bietet sich etwa in der DVD von Paramount (2008, Region 1) im Top-Menü zunächst die Option, aus den in einem ovalen Kreis angeordneten (gegen den Uhrzeigersinn laufenden) Schriftzügen Play - Scene Selection Theatrical Trailer - Set Up zu wählen (Abb. 7.12). Wählt man Play, ${ }^{47}$ erhält man nebeneinander die Optionen VIEW MOVIE WITH ONE OF ITS THREE THEATRICAL ENDINGS (AT RANDOM) oderVIEW THE HOME VIDEO VERSION OF THE MOVIE WITH ALL THREE ENDINGS (Abb. 7.13).

Abb. 7.12

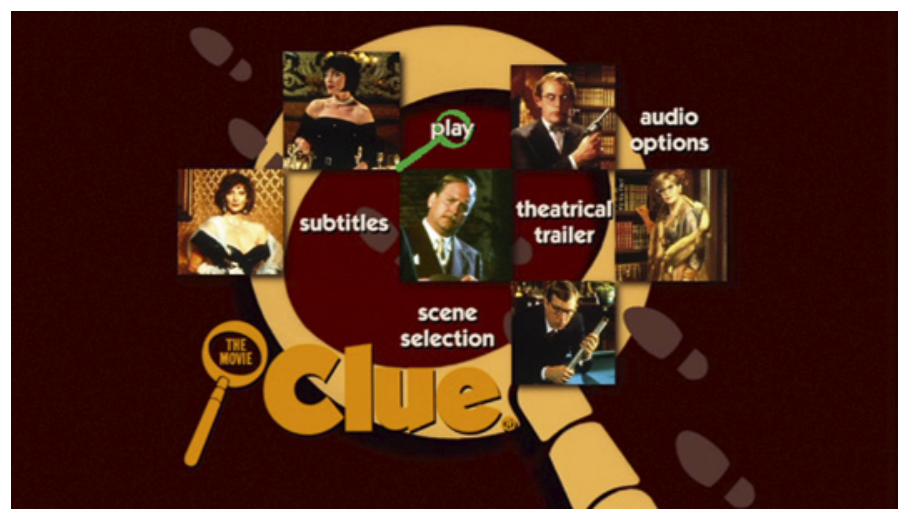

46 Gleichzeitig weckt der Begriff, Red Herring“ Assoziationen an die Auseinandersetzungen in der McCarthy-Ära um ,rote', das heißt kommunistische Umtriebe in den USA.

Zur Form des Icons s. unten. 


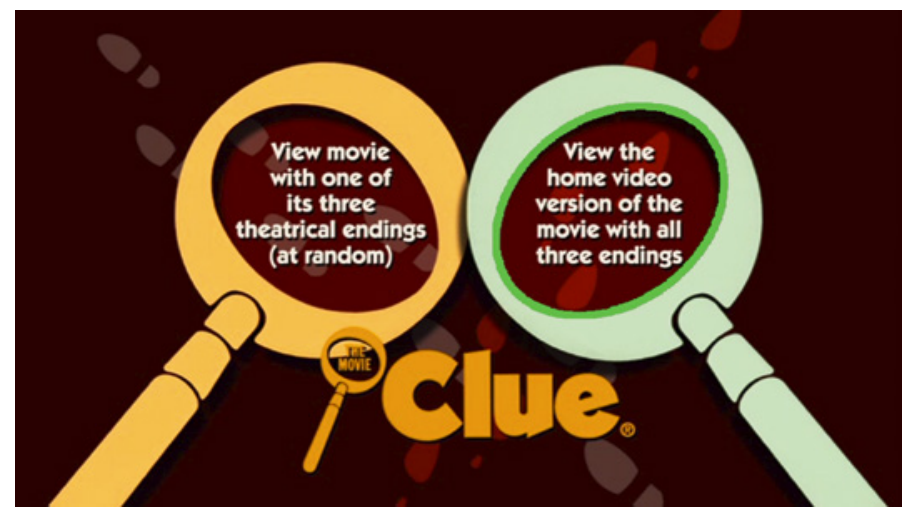

Hat man sich vor dem Schauen des Films für die zweite Option entschieden, so wird nun, als Wadsworth nach der Verdunkelung des Bildes zur finalen Aufklärung ansetzt, tatsächlich zunächst eine Version - mit handlungswiederholenden Rückblenden - erzählt. Wenn diese Version endet, erscheint ein Zwischentitel im Stil der Stummfilmpraxis: „That's how it could have happened“. Dann ein nächster Zwischentitel: „But how about this?“ (Abb. 7.14-7.15).

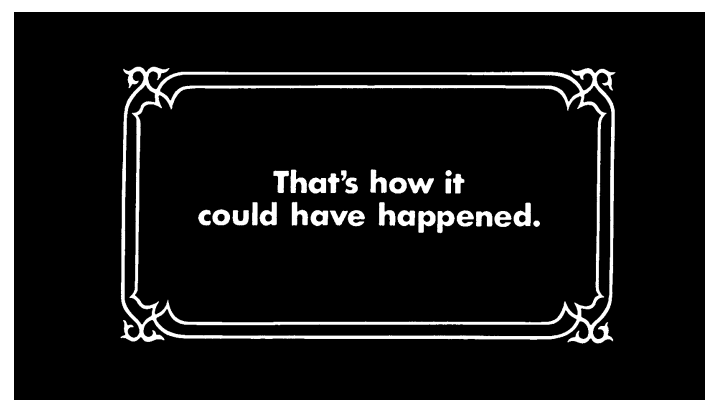

Abb. 7.14

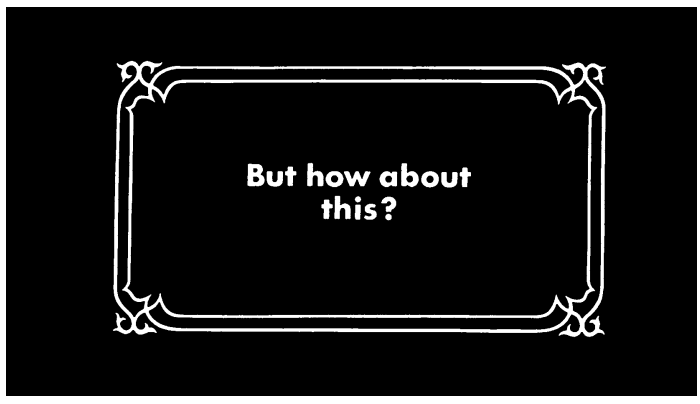

Abb. 7.15 
Danach wird - erneut bei dem Wiederanschalten des Lichts beginnend - das zweite Ende mit einem anderen Täter erzählt. Nach dieser Version folgt erneut ein Zwischentitel: „But here's what really happened“. Dieser Zwischentitel überschreibt die vorher in der Kinodistribution gegebene Koexistenz verschiedener gleichberechtigter Lösungen und privilegiert inhaltlich das Ende, in dem alle Figuren an den Morden beteiligt sind. Damit wird - im Gegensatz zu dem, was Hartmann noch als Potenzial einer Forking-path-Struktur betont - das Spiel mit der multiplen Möglichkeitsdimension der Geschichte und mit der Versatilität des fiktiven Universums zurückgenommen. Allerdings nur in dieser Version. Betrachtet man das Dispositiv DVD, offeriert es ja gleichberechtigt noch die Möglichkeit, den Film mit nur einem der drei Schlüsse über eine Zufallsfunktion zu sehen. Insofern stellt die DVD/Blu-Ray-Disc in der Vervielfachung der Optionen eine Nähe zur ursprünglichen Distributionspolitik im Kino her - auch wenn der Raum der Filmerfahrung zwischen Kino und der Rezeption zu Hause über einen Heimkinoträger sich nachhaltig unterscheidet.

Insofern wäre die Edition des Films nach Distelmeyer (2012), der allerdings den Fall von Clue nicht ausführlich analysiert, ein Paradebeispiel für die Konvergenz von Brettspiel, DVD und digitalem (Computer-)Spiel, definiert über die Interaktivitäts- beziehungsweise Wahlmöglichkeit des Nutzers/Users (in Abgrenzung zur Rezeptionshaltung im Kino). Die Tagline des Films weist fast prophetisch auf diesen intermedialen Kreislauf hin: Clue. The Movie - It is not a game anymore. Dies vielleicht umso mehr, als das Menüdesign (dies gilt sogar für verschiedene Editionen) sich mit dem Genre der Detektivstory verbindet und das Thema der medialen und zeichenhaften Dechiffrierbarkeit der Welt omnipräsent ist. Besonders augenscheinlich wird dies bereits in der Gestaltung des Menüs, in der die Lupe als optisches Gerät das Auswahl-Icon darstellt. Des Weiteren sind auch die gezeigten Filmstills von Lupen gerahmt. Dies lässt sich auf der Metaebene als eine symbolische Problematisierung der Dechiffrierbarkeit der Filmbilder lesen.

Der Film, sein fiktives Universum, spielt mit einem Überfluss von flottierenden Zeichen und Zitaten, wodurch eine verlässliche Interpretation von Spuren innerfilmisch sowie auf der metafilmischen Ebene infrage gestellt wird. Dies gilt auch in kulturhistorischer Perspektive: Der Film stellt narrativ wie formalästhetisch seine genretypischen Funktionsmechanismen aus. Er hat bereits inhaltlich ein pasticheartiges, spielerisches Verhältnis zu seinen literarischen, filmischen und auch theatralen Vorläufern - man denke etwa an The Mousetrap (1950) von Agatha Christie.

Das Dispositiv der DVD begradigt augenscheinlich zunächst die eigentlich zeitgenössische Versionenhaftigkeit des Films in Hinblick auf sein Ende - in der 
Weiterschreibung der Überlieferungsvariante der VHS, die über die Montage und die Ergänzung der Zwischentitel ein Ende zur finalen Auflösung erklärte.

Allerdings sind doch die Eindrücke in der Erfahrung der Dauer des Films widersprüchlicher. Hier prägen sich insbesondere der unbekümmerte und kontinuierlich spielerische Umgang mit jeglicher Form von Bedeutungsproduktion im gesamten Filmverlauf und die an den Slapstick angelehnte Darstellungsform ein. Unter diesen Vorzeichen tritt eine auf die Narration fokussierende Filmwahrnehmung, die sich auf das kognitiv zu lösende Rätsel des Whodunits konzentriert, in den Hintergrund. Ganz im Sinne eines allgemeinen Verständnisses von ,Spiel ' wird aus Lust an der Unterhaltung und an dem (Wieder-)Erkennen von Situationen die Frage, wer den Mord eigentlich begangen hat, sekundär. Vielmehr entwickelt sich ein Spektakel, in dem die Performanz von potenziellen Bedeutungsdimensionen ostentativ ausgestellt wird. Der Zweck, den Mörder zu finden, relativiert sich im Verhältnis zum Spaß an der Möglichkeit, es könnte sogleich immer alles ganz anders sein.

Insofern verstärkt die DVD diesen Charakter - auch weil sie in die dispositivische Anordnung verlegt, dass die Geschichte per RANDOM FUnKTION auch anders ausgehen könnte: Wenn man denn diese Funktion wählt. Dass aber die Home Video Version und die RANDom-Version beide auf einem Medium vereinigt werden, welches man im privaten Raum rezipiert, kreiert einen besonderen Modus der Aneignung, der sich mit der Versatilität des Filmwerks verbindet. Das Potenzial zur Wahl der Alternative kann eine Praxis der wiederholten Rezeption des Films befördern.

Vor diesem Hintergrund erklärt sich dann auch der nachträgliche Erfolg des Films in Dispositiven jenseits des Kinos, in denen die Verfügbarkeit im heimischen Raum gegeben ist. Dies hatte seinerseits wiederum eine interaktive Metarezeption zur Folge, die in der Öffentlichkeit des Internets ausgetragen wurde: „Thanks to cable re-runs and incredible word of mouth, however, the film slowly built a giant following to the point [that, $\mathrm{FH}$ ] random Internet outlets were writing overly long stories about its endings three decades later" (Rawden 2015).48 Grundlage dieser Metarezeption war - so mein Befund - die

48 H. Jenkins (1992: $68 \mathrm{ff}$.) weist mit Blick auf fankulturelle Praktiken bereits medienhistorisch auf die Bedeutung von Videorecordern (VCR) hin. Hier sind insbesondere die Möglichkeiten der Aufnahme und der ,Kontrolle über die Rezeptionsformen von Filmen und von TV-Serien zentral, wodurch auf unterschiedlichen Ebenen Aneignungsmodi im privaten Raum wirksam werden. Auch Wiederholungen im Fernsehen sind für Formen der Aneignung wichtig (was an meine Überlegungen zum Abschluss von $\rightarrow$ Kap. 6 anknüpft). Nach H. Jenkins werden über diese medialen Praktiken Re-Readings, das heißt Bedeutungsmodulationen, forciert. Der Videorecorder als ein Vorläufer digitaler Nutzungs- und Rezeptionsformen habe so eine Verfügungsmacht der Rezipienten über 
Möglichkeit des Vergleichs der drei editierten Enden, den die Heimkinoversion ermöglichte. Im heimischen Dispositiv arbeiteten der Aneignungsgestus des Vergleichens und die freiere - im Vergleich zum Kino weniger disziplinierte Rezeptionshaltung (Hansen 1993) - der spielerischen Erzählstruktur des Films zu.

Für die Vorstellung von Filmgeschichte bedeutet dieser neu gewonnene Kultstatus des Films, dass heute meist die Fassung für das Heimkino als Referenz betrachtet wird - allein durch die Praktiken der Zugänglichkeit. Insofern hat sich diese dispositivisch angepasste Version in die Überlieferungsgeschichte nachdrücklich eingeschrieben. ${ }^{49}$ Der Modus der Aneignung über spielerische Formen des Vergleichens (der Enden) gehört nun zur einkalkulierten Rezeptionshaltung des Films. So realisiert sich etwa auch im vom Dispositiv offensiv provozierten seriellen Mehrfachschauen, im aktiven Suchen von Differenzen und Wiederholungen seitens des Zuschauers der auratisierende Mehrwert von Clue. The Movie, der maßgeblich das heutige Prestige und den Status des Films begründet.

In wahrnehmungstheoretischer Perspektive gerät das Filmwerk zu einem medialen Cluster, in dem die gegenwärtig rezipierte Version immer schon mit ihrer potenziellen Alternative im Erlebnis des Zuschauers koexistiert. Dieses spezifische Erlebnis im Falle von Clue muss im Zusammenspiel mit dem Filminhalt und seiner Wirkungsästhetik gesehen werden: Mediale Zeichenhaftigkeit wird als performatives Zitatespiel der Kultur- und Mediengeschichte vermittelt. Damit birgt der Film schon an sich die Erfahrung der spielerischen Relativierung von verlässlich dechiffrierbaren Bedeutungen und Zeichen; je nach getroffener Wahl etablieren sich nur situativ augenblickhaft Sinnzusammenhänge. In Wechselwirkung mit dem Dispositiv DVD, in dem die Distributionsgeschichte in einem Navigationsraum konfiguriert und als interaktiv auswählbar präsentiert wird, bedeutet dies einen Erfahrungshorizont von zeitlichen Differenzen, der in hohem Maße die Erinnerungsaktivität und zugleich körperliche Präsenz des Zuschauers (ein)bindet.

Forciert durch ein (digitales) Dispositiv, das Kontrolle und Aneignung von Filmen suggeriert, erhält diese zeitliche Komponente auch in der möglichen Serialität des Rezeptionserlebnisses eine gewichtige Rolle: Barbara Klinger (2006a) markiert hier - wie bereits oben erwähnt - die Verbindung

die filmische Erzählung ermöglicht: „These viewing strategies, made possible by the technology's potentials, extend the fans' mastery over the narrative and accommodate the community's production of new texts from the series materials" (H. Jenkins 1992: 74).

49 Eine neue Verfilmung des Spiels ist angekündigt (Stand 2019). Gleichzeitig gab es auch eine TV-Miniserie mit fünf Episoden, die 2011 in den USA lief. 
von strukturellen Merkmalen des puzzle films und den dispositivischen Implikationen des Heimkinos (2006a: $135 \mathrm{ff}$.). Sie hebt insbesondere den Zusammenhang von Anreizen, die solche Filme als Interaktion mit dem Zuschauer einfordern, ${ }^{50}$ und dem möglichen mehrfachen Schauen über Heimkinotechnologien im aneignenden Modus des privaten Raums hervor (Klinger 2006a: 157). Dies habe für den Rezipienten beziehungsweise Nutzer zur Folge, dass er funktional zu verstehen sei als

a savvy decoder of a text's mysteries, the viewer becomes something of an authority - an intrepid explorer who has discovered a terra inocgnita. [...] Mastery is a steadfast component of home film reception in the digital era, given to different articulations by the discourses that seek to define domestic space as a special viewing territory. Here, the pleasure of repeat viewing lies in the viewer's ability to unearth visual details and layers of meaning that cannot be fully apprehended in initial screenings (Klinger 2006a: 161, Hervorh. FH).

Klinger verschiebt die Vorstellung von der Autorität der Industrie - oder eines hochstilisierten Autors - über das mediale Produkt hin zum Konzept von filmischen wie dispositivischen Rahmungen, die den Rezipienten im häuslichen Kontext des Filmeschauens zum Koautor und zur machtvollen Instanz über die medialen Phänomene werden lassen. ${ }^{51}$

Allerdings - und dies ist nun ein entscheidender Unterschied zwischen Klingers Überlegungen und meinen - geht Klinger dabei vom wiederholten Schauen ein und desselben Films zu Hause aus, nicht von unterschiedlichen Versionen. ${ }^{52}$ In dem vorliegenden spezifischen Zusammenhang beschränkt sich aber die Vorstellung des zu lösenden Puzzles nicht nur auf den zu entschlüsselnden Inhalt eines Films, sondern darüber hinaus auf die jeweiligen Formen der Mise en Relation der (historischen) Versionen in den digitalen

50 "The idea that a film cannot be adequately consumed on the first viewing is the bedrock proposition of aesthetic motivations for re-viewing“ (Klinger 2006a: 157).

51 Hier sei weiterführend auf Begriffskomplexe im Diskurs um Digitalkulturen verwiesen: das Konzept des Produsers beziehungsweise der Produsage (Bruns 20o8). In diesem Horizont verschwimmen die Grenzen von Produktion und Rezeption, und Fragen der hegemonialen Bedeutungsproduktion stellen sich neu. Hier ließe sich mit Rekurs auf Manovich (2001) eine ambivalente, durchaus kritische Sicht der den Nutzer ermächtigenden Wirkung von digitaler Interaktivität weiterdiskutieren - ähnlich wie es etwa schon Distelmeyer (2012) mit Blick auf die ideologischen, machtstrukturellen Implikationen der Programmierung von Wahlmöglichkeiten („Spiel der Macht“, 2012: u. a. $47 \mathrm{ff}$.) einer DVD beziehungsweise Blu-Ray-Disc ausführlich getan hat.

52 Mit Blick auf die digitale Ära sieht Klinger dies besonders bei Filmen von Steven Spielberg und George Lucas gegeben, vor allem bei Filmen mit Fokus auf Special Effects und mit Blick auf eine (damit) vervielfachte, kompliziertere Bildebene, die zu einer wiederholten Rezeption in der heimischen Sphäre einlädt (Klinger 2006a: 161). 
Dispositiven. Damit enthält meine Perspektive die Frage nach den sich hier entfaltenden Aspekten der medienhistoriografischen Wirksamkeit, die nicht losgelöst zu sehen ist von den präsentischen Rezeptions- und Selektionsakten des Zuschauenden/Nutzenden. Somit geht es in meiner Sicht nicht nur um die Beschreibung kognitiver Entschlüsselungsprozesse, sondern vor allem auch um die Konzeption leiblicher Erlebnishorizonte von zeitlichen Differenzen angesichts von Filmen und ihren Fiktionen in verschiedenen Versionen.

Filmästhetische und dispositivische Verunsicherung von

Wahrnehmung. Relativierung verlässlicher Subjektpositionen

Phänomene der Legierung von Filminhalt, -struktur und metafilmischer Fassungsgeschichte im Horizont digitaler Reprisen ${ }^{53}$ weisen weitere interessante Effekte auf, gerade wenn die editierten Filme selbst-reflexiv Bedeutungsproduktion über Bilder und Zeichen inhaltlich infrage stellen und dies ostentativ in ihrer Motivik, Ästhetik und Struktur umsetzen. Da die Filme oft auch am exponierten Beispiel der Gestaltung ihrer Protagonisten verlässliche Identitäten prekär erscheinen lassen, verhandeln sie damit auch grundsätzlicher das Verhältnis des Subjekts zur Welt.

Der Fall der Blu-Ray-Edition des Films Memento (Nolan, 200o) offenbart in diesem Kontext eine interessante Verschmelzung von Inhalt, filmischer Form und dispositivischer Warenästhetik. Grundsätzlich attestieren Kiefer/ Große (2006) dem Film Memento, dass er „die entscheidenden Merkmale des Film noir im instabilen Charakter des Protagonisten, der verschachtelten Rückblendenmontage, des Einsatzes der Voice Over [...] bis zur vollkommenen Verunsicherung treibt“ (Kiefer/Große 2006: 253).

In der wissenschaftlichen Rezeption stellt der Film ein paradigmatisches Exempel der narratologischen Diskussion um Strukturelemente des bereits genannten puzzle films dar (in Auseinandersetzung mit Bordwell 2006, vgl. bes. Buckland 2009: 5). Vor allem Ghislotti (2009: 104) hat den Film auf seine „mnemonic devices“ hin untersucht - sowohl auf der Ebene des Inhalts (dazu später mehr) als auch auf der Ebene der notwendigen Erinnerungsaktivität des Zuschauers angesichts der puzzleartigen Erzählform.

So stellt der Film Leonard Shelby (Guy Peirce) in den Mittelpunkt, der seine Frau durch einen Mord verliert. Bei der Tat fügt der Mörder Shelby eine schwere Kopfverletzung zu, die bei diesem zum Verlust seines Kurzzeitgedächtnisses führt. Trotzdem begibt Shelby sich auf die Jagd nach dem Mörder.

Intermediale und selbst-reflexive Bezüge sowie Versuche, über Medien der Welt und der eigenen Geschichte habhaft zu werden, sind allgegenwärtig in

53 Zum digitalen Dispositiv von Streamingdiensten vgl. den Abschluss von $\rightarrow$ Kap. 7 . 
dem Film. Shelby benötigt mediale Erinnerungsstützen wie Fotografien und Niederschriften auf Papier. Darüber hinaus benutzt er selbst seinen eigenen Leib, konkret seine Haut, als letztes Mittel der Selbst-Vergewisserung, um über Tätowierungen als Einschreibungen sich seines Körpers zu versichern.

Besonderes strukturelles Merkmal des Films ist der komplexe Aufbau der Zeitstruktur, weil sich Schwarz-Weiß-Bilder, die in einer fortschreitend chronologischen Ordnung montiert sind, mit farbigen Sequenzen verweben, die in umgekehrter Logik geschnitten sind und damit die Amnesie des Protagonisten vermitteln. Auf diese Weise entsteht ein Weltbild, in dem Shelby nichts aufklären kann,

sondern [in dem sich, FH] nur die Brüchigkeit und Unzuverlässigkeit einer subjektiven Weltwahrnehmung offenbaren. MEмEnTo überführt die detektivische Suche nach komplexer Wahrheit in die Frage nach Illusion und Konstruktion im Akt des Erinnerns (Kiefer/Große 2006: 253).

Damit unterminiert Memento - so Kiefer/Große - alle Gewissheiten des filmischen Erzählens, der Motivationen von Handlungen, der erinnernden Vergegenwärtigung der, wahren' Ereignisse und am Ende sogar die personale Identität des Erzählers. So ist der Zuschauer herausgefordert, selbst Sinnzusammenhänge zu konstruieren (2006: 255-256). Die plurale Montage unterschiedlicher Wahrnehmungs- und Interpretationsmuster bindet den Zuschauer wirkungsästhetisch nachhaltig ein: „The whole film is immersed in the past, and we can say that it is entirely based upon a memorial dimension, structured and reconstructed on the basis of the viewer's memory of selected images" (Ghislotti 2009: 105).

Die Dispositive von DVD und später auch Blu-Ray-Disc-Editionen (u. a. Columbia Tristar, 2002 $)^{54}$ verstärken diese im Film angelegten strukturellen Elemente weiter. Damit, so meine These, erklären sie den Film an sich als unverlässlich - in seinem Status als Werk und als Text. Dies wird in bestimmten Editionen mit einem Entdeckungsgestus verbunden, dass man einen Pfad im DVD-/Blu-Ray-Disc Menü erst finden muss, um den Film in einer anderen Version sehen zu können. Zu einer solchen zusätzlichen Funktion der DVD und der damit eingeforderten Rezeptionshaltung beziehungsweise dem entsprechenden Nutzerverhalten schreibt Distelmeyer: „Easter Eggs variieren die hypermediale Struktur der Menüs nur insofern, als die gelegten Links beziehungsweise ihre Zeichen im Interface erst noch gefunden, das heißt: als

Vgl. DVD-Forum zu Memento/EAster EgG (2002) $\rightarrow$ Literaturverzeichnis. 
solche erkannt werden müssen“ (Distelmeyer, 2012: 118). ${ }^{55}$ Distelmeyer spricht in diesem Kontext mit Blick auf Memento von einem „berüchtigten“ Easter Egg (Distelmeyer 2012: 118), in dem der für seine nonlineare Montage berühmte Film in der sukzessiv chronologischen Schnittfolge präsentiert werde. ${ }^{56}$ Zunächst erscheint dies als eine Variante der Konsekrationsstrategie nach Bourdieu (2011c: 101-102), in der der DVD-Nutzer als Entdecker einer sonst ungenutzten Ressource in Szene gesetzt wird, wodurch - im Zusammenspiel mit der „Rhetorik der Intimität" von Insiderwissen (Klinger 2006a: 89) - Eindrücke von Prestige und symbolischem Mehrwert erzeugt werden sollen. Doch ist der Fall der digitalen Reprisen von MEмEnTo wirkungsästhetisch komplexer.

Über die motivische Gestaltung und Ästhetik der Menüs wird die Problematisierung der Entzifferbarkeit der Welt aufgegriffen. Vor allem werden Polaroidfotos zitiert, wobei die Augenblickhaftigkeit von Erscheinungen in der Inszenierung betont wird: ${ }^{57}$ Die Bilder und Schriftzüge gehen auf in einem Wechselspiel aus Erscheinen und Verblassen (Abb. 7.16).

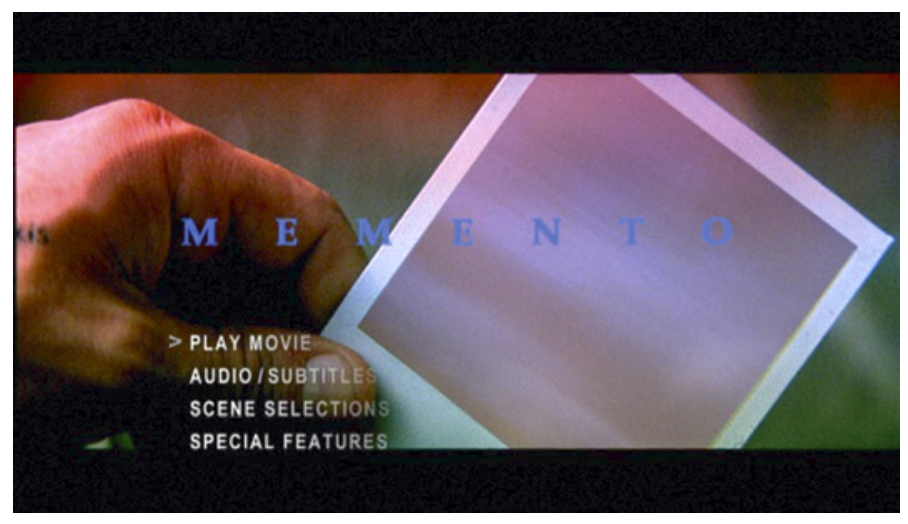

Abb. 7.16

55 An dieser Stelle ließen sich auch fruchtbar Ansätze zum Hypertext beziehungsweise zur Hyperfiktion anschließen; etwa Überlegungen, ob diese Formen der verlinkten Erweiterung des filmischen Textes nicht eine hyperfiktionale Variante des diegetischen Universums darstellen.

56 Dies veranlasst Distelmeyer zu einem neuen qualitativen Urteil über den Film. In dieser chronologisch erzählten Version werde die Ansammlung von Klischees, die eigentlich in dem Film bereits vorhanden sei, aber aufgrund der Montage verdeckt, nun unverstellt sichtbar. Es wäre sicher interessant, diesem Urteil filmanalytisch weiter nachzugehen; im gegebenen Kontext aber sollen Formen und Effekte der Mise en Relation im Fokus stehen. Vgl. zur augenblickhaften Passagenhaftigkeit von Erinnerungen nach Benjamin: Scherer (2001) und Czekaj (2015); $\rightarrow$ Kap. 3, $\rightarrow$ Kap. 5 . 
Der Zugriff auf den Film wird dem Nutzer bereits in einem instabilen Universum präsentiert. Die strukturell notwendige Handlung zum Entziffern der Zeichen und zum Aufspüren des Easter Eggs vollzieht der Nutzer in einer auf ähnlichen Wahrnehmungsprinzipien basierenden Spurensuche, wie sie der labile Protagonist unternimmt. Interessant ist die Dopplung: Der Protagonist ist amnesisch, auf der Suche nach der eigenen Erinnerung, und damit wird seine Weltwahrnehmung, die uns filmisch erzählt wird, unverlässlich. Mediale Bilder sind in diesem Universum nur trügerische Versprechen auf Bedeutung. In eine vergleichbare Wahrnehmungsdisposition wird der Nutzer versetzt, begibt er sich auf die Suche nach den auf der Film-DVD tiefer liegenden Geheimnissen. Für die Wahrnehmung des editierten (Haupt-)Films gilt aber damit auch, dass der Status der filmischen Fiktion als Werk mit spezifischen Strukturelementen in dieser Form der Mise en Relation als nicht fixiert wahrgenommen wird. Im Dispositiv der DVD steht er zumindest potenziell zur Disposition. Wichtig ist zudem - darauf weist auch Distelmeyer hin, der im Übrigen die Wahrnehmungsdisposition dieser spezifischen Edition nicht thematisiert -, dass es oft zum Entdecken solcher Easter Eggs der entsprechenden Seiten im Internet als Hinweisgeber bedarf, damit der Recherchegestus intermedial in eine breitere Öffentlichkeit getragen wird. ${ }^{58}$

Filminhalt, seine (interaktive) Zugangsform, die Ästhetik des Menüs und dispositivische Konventionen (zu entdeckende Easter Eggs und alternative Versionen als warenästhetischer Mehrwert einer DVD) sowie damit einhergehende, mittlerweile ritualisierte Metadiskurse (über die Institution entsprechender Webseiten) prägen das Werk als zeitlich heterogenes Cluster.

Dabei kreiert das Cluster einen Erwartungs- wie Erfahrungshorizont, der auf unterschiedlichen Ebenen mit der destabilisierenden Wirkung einer sich nur augenblickhaft vermittelnden Erkennbarkeit arbeitet. Damit werden Subjektpositionen als abhängig von zeitlicher Mise en Relation inszeniert: Die besondere Rolle der Zeit und der Erinnerung, die in dem Film verhandelt wird und sich als wirkungsästhetisches Prinzip umgesetzt sieht, wird im digitalen Dispositiv auf die Zugriffsformen des Films als Werk übertragen. Der Effekt: Der Zuschauer verfügt zwar als Besitzer und Nutzer formal über die DVD,

$5^{8}$ Distelmeyer entwirft hier unter anderem eine Öffentlichkeit, die sich zum einen als populärkulturelle, cinephile Expertengruppe versteht; dies auch angesichts des Aneignungsgestus im Besitzen und Sammeln von Home-Video-Trägern; vgl. hierzu weiterführend: u. a. Wortmann (2010), Balcerzak/Sperb (2009 und 2012); zu einer Liste der möglichen entsprechenden Webseiten Distelmeyer (2012: 118). Zu Überlegungen von cinephilen Filmfans in Video-on-Demand-Medienumgebungen vgl. vor allem Hagener (2016) sowie kleinere Beiträge wie etwa VanDerWerff (2016). 
angesichts der ostentativen Subjektivierung von Zeit erscheint er verfangen und relativiert zwischen Erinnerung und Gegenwart.

Das digitale Cluster MЕмеNто ist darüber hinaus ein so interessanter Fall, weil es sich auch in den theoretisch-analytischen Diskurs um puzzle films methodologisch eingeprägt hat: Die auf das narratologische Konzept ausgerichtete, bereits zitierte ausführliche Analyse von Ghislotti (2009) arbeitet explizit mit der auf DVD-Editionen (u. a. Columbia Tristar 2001) als Bonusmaterial veröffentlichten Version des Films in der chronologischen Schnittfolge (Abb. 7.17; vgl. letzter Menüpunkt ganz unten ${ }^{59}$ ).

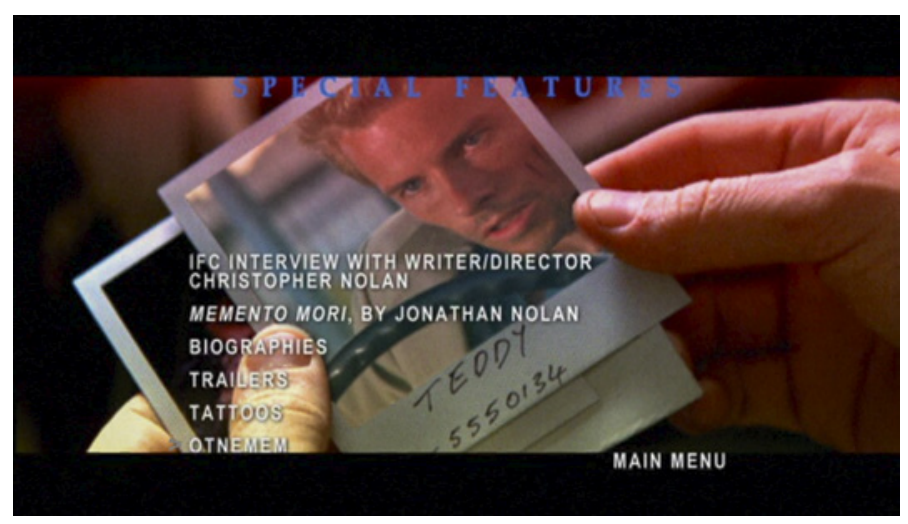

Abb. 7.17

Ghislotti unternimmt dies mit dem Anliegen, die komplizierte Fabula des Films analysierbar zu machen und die Bedeutung von Erinnerung in der umgekehrten Schnittfolge profilieren zu können. ${ }^{60}$

Bei Ghislotti (2009) wird allerdings die konkrete Zugangsform über das digitale Dispositiv und die Positionierung als paratextuelles ,Bonusmaterial ' zumal wie oben ausgeführt - in ihren formierenden Konsequenzen kaum thematisiert und mitberücksichtigt. Vornehmlich die Zugänglichkeit einer anderen Version wird bei Ghislotti zum analytischen Tool des narratologischen Erkenntnisprozesses im Modus des Vergleichs. Die dispositivischen und

59 Bemerkenswert ist hier, dass der Menüpunkt, unter dem man diese Version anwählen kann, mit OTNEMEM betitelt ist.

6o „As an effect of the backward narration it is very difficult for the viewer to have a clear idea of what actually happens. We'll try to understand how this effect is obtained by comparing two versions of Memento: the original release, screened in movie theaters, and the chronological version, provided as bonus material in the DVD edition. We have to deal firstly with the chronological version, the film that many viewers were probably expecting during the screening of the actual version of MEMENTO" (Ghislotti 2009: 90). 
ästhetischen Bedingungen, die diesen Vergleich allerdings erst möglich machen und die das rezipierende Subjekt in seiner Zeitwahrnehmung des Films selbst formieren wie funktionalisieren, werden weitgehend ausgespart.

Kurz sei vor diesem Hintergrund schließlich auf eines der bekanntesten Beispiele hingewiesen, das sowohl in der Kinodistribution als auch in der begleitenden Home-Video-Distribution zahlreiche Versionen hervorgebracht und damit in der Folge den wohl bekanntesten populärwissenschaftlichen Metadiskurs über Filmversionen kreiert hat. Die Rede ist von BLAdE RUNNER (Scott, 1982). Sowohl in wissenschaftlichen Publikationen (vgl. bes. ausführlich Relinger 2009, aber auch Bohn 2013b: $391 \mathrm{ff}$.) als auch auf verschiedenen Internetseiten (vgl. etwa Schnittberichte oder DVD-Forum.at ${ }^{61}$ ) finden sich entsprechende Dokumentationen und auch Illustrationen mit eigenen vergleichenden Bildserien und -gegenüberstellungen. Im Folgenden soll es vor allem um die Five Disc Ultimate Collector's Edition (Warner Bros., 2007) als Box gehen. Hier gilt nicht mehr, dass mehrere Versionen auf einem digitalen Medium Platz finden, sondern die Versionen werden diesmal kompakt zusammen editiert in einer ganzen Box, die beworben wird mit dem Slogan „erstmals fünf Versionen des Films zusammen“62 (Abb. 7.18).

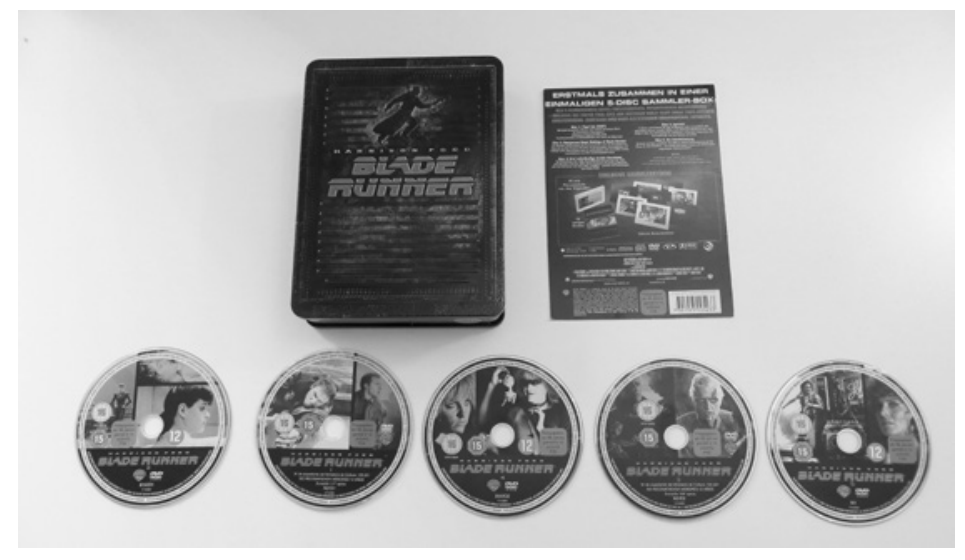

Abb. 7.18

$61 \quad$ DVD-Forum.at zu BLADE RUNNER (2010).

62 "Given the different versions of BLADE RUNNER that have been available over the years, including them all in a single disc release is a challenge. Thankfully, Warner has used the seamless branching capabilities of DVD, Blu-ray Disc and HD-DVD to include all three legacy versions of the film on this one disc. Available here are the 1982 U.S. Theatrical CUt (117 minutes), the 1982 International Cut (118 minutes) and the 1992 Director's CUt (117 minutes). You simply select the one you want from the disc's opening menu. That takes you to a submenu where you can select the various options, start the film or watch Scott's introduction (available for each version)“ (B. Hunt 2007). 
Damit greift die Box die bisherige Fassungsgeschichte auf, denn sie enthält Versionen, die jeweils verschiedenen Normierungsprozessen unterlagen - ob ökonomisch motiviert, als Reaktion auf Äußerungen eines Testpublikums verändert, ob auf Grund geopolitischer kultureller Anpassungen („domestic and international versions"), über Formatnormierungen (Fernsehfassung) oder über die von einer Auteur-Politik getragenen und zugeschnittenen Versionen. Namentlich sind dies die folgenden Fassungen: Work Print, SNeak-PeaKVersion, Theatrical US-Version, Theatrical International (1982), Broadcast (1986), Director's Cut (1992), Final Cut (2007) (vgl. zu den Entstehungsgründen auch Bohn 2013b: $391 \mathrm{ff}$.). Insbesondere die formalen wie inhaltlichen Differenzen zwischen der 1980er Kinofassung und dem Director's Cut haben zu vielen öffentlichen Diskussionen geführt, weil sich - neben der Darstellung von Gewalt - vor allem folgende Variationen beobachten lassen:

a. Die Geschichte und die moralisch-ideologische Botschaft ändern sich; dies insbesondere durch das alternative Ende und das Einfügen einer Sequenz, die eine Schlüsselfunktion für die Identität des Protagonisten hat.

b. Insofern gilt auch, dass sich die Problematisierung der Erkennbarkeit und Verstehbarkeit der Umwelt je nach Version unterscheidet. Dies gilt umso nachhaltiger für die jeweilige Auseinandersetzung mit Fragen nach der Verlässlichkeit des (erzählenden) Subjekts und mit den Relativierungen von Identitäten. Dies geht mit strukturellen Variationen der formalästhetischen Genreelemente des Film Noir einher, unter deren Maßgabe in der ersten Kinofassung das Verwenden einer Voice-Over des Protagonisten erfolgte, die für die Filmwahrnehmung inhaltliche und atmosphärische Konsequenzen zeitigte.

c. Die Schnittrhythmik und dadurch die schon angesprochene atmosphärische Wirkung variieren zum Teil signifikant in den einzelnen Versionen. Worum es vor diesem Hintergrund gehen soll, ist die implizite serielle Filmerfahrung, die die BLADE-RunNER-FIVE-DisC-COLlECTOR's-Box offeriert und die ich als zeitliches Differenzerlebnis und als medienhistoriografisch wirksam begreife. Alle fünf Fassungen werden zusammen unter dem Titel BLADE RUNNER räumlich in der Box aufeinander bezogen angeboten. Man kann also eine Version linear rezipieren, danach in einer Variation - so das warenästhetische Versprechen des Dispositivs. Sollte man den Modus des wiederholten Schauens wählen, so manifestiert sich eine womöglich nur nuancierte Differenzerfahrung (v. a. Voice-Over, Ende, einige eingefügte Einstellungen), die sich aus der subjektiven Erinnerung an eine andere Version speist (geht man von einer normalen Rezeptionssituation aus: ohne einen zusätzlichen Aufbau von zwei Bildschirmen, der eine Parallelsichtung möglich machen 
würde). Die Filme lassen sich nur hintereinander anschauen, dispositivisch wie etwa im Beispiel zum Editor's Guide bei Close Encounters of THE THIRD KIND - wird kein direkter Vergleich angeboten. Aber durch die editorische Mise en Relation der Box ergibt sich die potenzielle und warenästhetisch suggerierte Serialität. Dieses Potenzial hat wieder die Überlagerung der Wahrnehmungsebenen zur Folge. Es verweben sich thematische wie wirkungsästhetische Angebote, die der Film(text) selbst macht, welche sich aus einer dystopischen Film-Noir-Welt speisen und nachhaltig das Motiv des Auges thematisieren und dabei die Unterscheidbarkeit von Realität, Traum und Simulation infrage stellen. In Verbindung mit der Koexistenz der Versionen im digitalen Dispositiv der DVD/Blu-Ray-Disc erhält der Film als definierter Text zusätzlich einen unsicheren Status - selbst wenn gewisse Hierarchien durch Benennungen („Director's CuT“ oder „Final Cut“) sowie durch Nummerierungen über Menüanordnungen nahegelegt werden. Die Bedeutungsdimension des an sich schon ambivalenten Films wird über die Editionsform dennoch erweitert. Sollte man einen solchen seriellen, wiederholenden Rezeptionsmodus wählen, formuliere ich mit Blick auf die konkrete Filmerfahrung die These, dass die narrative Dimension des Films (Was passiert ...?) in diesem Fall zurücktritt zugunsten eines graduell verdichteten Erlebnisses von atmosphärischen und rhythmischen Elementen der entsprechenden Version. Die Lust entsteht im Modus des sinnlich erlebten und erinnerten Bildervergleichs.

Das kulturelle Prestige und die sinngebende Autorität verschieben sich somit generell über die Nutzungs- und Rezeptionsform in das Erlebnis der subjektiv empfundenen Dauer und der Erinnerungsaktivität. Ideell und memotechnisch, aber auch ganz konkret auf der praktischen Ebene wird der Nutzer/Konsument zum bewahrenden, sammelnden Archivar (auch nahegelegt durch die Betitelung der Box als Collector's Edition). ${ }^{63}$ In diesem Zusammenhang betont erneut die Relativierung des einen Werkes - auch als Rezeptionserfahrung - paradoxerweise den kulturellen Wert des Clusters Blade RunNer.

Dies kann ergänzt werden durch die Kenntnisnahme des im Internet geführten Metadiskurses, der sich faktografisch der Ergründung der Varianzen

63 Für weitergehende Analysen von Fankulturen stellt in diesem Kontext ergänzend der Zusammenhang von Cinephilie und Konsekrationsakten einen fruchtbaren Diskurskreis dar; vgl. hierzu generell Valck/Hagener (2005). Hier ist für meine Argumentation insbesondere der Beitrag von Robnik (2005: 55-64) zu „Mass Memories of Movies“ anschlussfähig; oder auch grundsätzlicher zur digitalen Kultur Balcerzak/Sperb (2009 und 2012). 
und der Suche nach Fixierung einer zeitüberdauernden, definitiven Version verschrieben hat (vgl. exemplarisch B. Hunt [2007] oder Moviecensorship.com zu BLADE RUNNER; $\rightarrow$ Literaturverzeichnis).

Verallgemeinernd lässt sich sagen: Begreift man darüber hinaus die einzelnen Versionen (hier konkret von BLADE RUNNER) als wirkungsästhetisch funktionierende sinnliche Puzzleteile, entstanden aus bestimmten historischen, ökonomischen, ideologischen und medialen Kontexten heraus, so konstituiert der Zuschauer über die serielle Erfahrung zwischen Differenz und Wiederholung seine Geschichte des Films - das heißt, diese Form von subjektiver Filmgeschichte wird ohne historische Kontextualisierung als präsentische Filmdifferenzerfahrung erlebt; über den Modus des Vergleichs, in dem die Erinnerung an andere Filmerlebnisse eine zentrale Rolle einnimmt, wird eine eigene, autobiografische Versionengeschichte kreiert. Die Unterhaltung und die Lust basieren hierbei auf dem Prozess der subjektiv erfahrenen Zeit, der Dauer im Bergson'schen Sinne. Selbst wenn diskursiv von den Produzenten beziehungsweise den Herausgebern eine Fassung zur ,finalen' Version erklärt wird, bleibt zumindest für die Wahrnehmung das Potenzial der Variation bestehen.

Zudem wird das Filmerlebnis mit weiteren Anreizen des Begehrens aufgeladen: Wie in den Ausführungen in $\rightarrow$ Kap. 4 mit H. Böhme ausführlich erläutert, geht damit eine bestimmte fetischisierende Rezeptionshaltung und (räumliche) Anordnung der fetischisierten Objekte einher. Dies hat die folgenden Implikationen:

1. Durch das spezifische Dispositiv, das heißt hier das Angebot einer Sammlung möglichst vieler alternativer Versionen, wird der Film mit einer besonderen kulturaffinen Überhöhung wahrgenommen, welche seiner nichtalltäglichen, herausgehobenen Attraktion gilt, die eben in diesem spezifischen Kontext der Box-Edition darin liegt, (historisch) variant zu sein.

2. So verursacht der als versatil verstandene Film eine auf potenzielle Alternativen angelegte Wahrnehmungshaltung, die zwischen dem Begehren nach Aneignung und der Faszination einer sich immer in der Alternative entziehenden Qualität oszilliert.

3. Durch ein Wechselspiel zwischen Nähe und Ferne wird der Film zum Gegenstand von Lust: Zum einen muss die Überhöhung zementiert werden, das Werk muss also möglichst schwer fassbar bleiben; gleichzeitig speist sich aber die Lust aus der Möglichkeit des Erlebnisses von Nähe in der Aneignung (etwa des wiederholten Schauens). Dieses Wechselspiel wird über die gemeinsame Edition, die räumliche Bezogenheit über die Box als besondere Anordnung wirksam (vgl. Dispositive des Fetischs, 
Kap. 4). ${ }^{64}$ Mit H. Böhmes Worten: „Die unbedingte Distanz bei gleichzeitig aufrechterhaltener Performanz des Fetisch verwandelt diesen in ein unverfügbares Element einer Erfahrung, die der Betrachter nur mit sich selbst machen kann“" (H. Böhme 2012: 357).

Insofern wird hier im Horizont der Mise en Relation von filmeditorischen Praktiken ein auf der medialen Erfahrung basierendes Konzept von Filmhistoriografie entworfen, das eine Referenz und einen Bezug auf die außermediale Ereignisgeschichte weit in den Hintergrund treten lässt. Dies obwohl doch die Ursachen der Varianz in verschiedenen historischen - technischen, ökonomischen oder ideologischen - Umständen begründet liegen. Das Werk BLADE RUNNER wird in einer heutigen, fetischisierten Zugangsform zum Cluster, dessen einzelne Schichten sich historisch deterritorialisiert präsentieren. Die historische Dimension findet allenfalls noch ihre Spur in dem präsentischen Wählen einer Version, dem potenziellen Erlebnis von Varianzen in einer zeitlichen Dauer; eine Form der zeitlich subjektivierten Filmhistoriografie, die sich lediglich augenblickhaft konstituiert und mit bewegtbildlichen, sinnlichen Erlebnisformen verbindet, ohne unbedingt eine außerfilmische Referenz zu denotieren. Interessanterweise ist dies ein Geschichtsbild, das dem Subjekt und seiner nicht verlässlichen Erinnerung einen zentralen Platz zuspricht und damit auch ein gesamtheitliches, in irgendeiner Weise fixierbares Weltbild negiert.

Die Anwendung des Begriffs der Filmhistoriografie ist im Kontext des Beispiels von BLADE RUNNER in meinem spezifischen Verständnis zu sehen vor allem auch in einem populären, auf die massenmediale Erfahrung hin ausgerichteten (vgl. hierzu die Erläuterungen zur Tragweite von Filmen als Formen der Erinnerungsbildung wie Historiografie in $\rightarrow$ Kap. 6). Diese Erfahrung gründet nur implizit auf geschichtlichen Konfigurationen, da sie sich vor allem in den Normierungsprozessen manifestierten und heute lediglich noch als Spuren in Form des Erlebnisses von (filmgestalterischen) Differenzen, die aus den historischen Produktions- und Distributionspraktiken resultieren, wahrnehmbar werden. ${ }^{65}$

64 H. Böhmes (2012: 355) Referenzanalogie ist hier das Vitrinenglas, hinter dem das Objekt in einem Museum ausgestellt ist. Das Vitrinenglas suggeriert zwar Nähe durch die mögliche Betrachtung eines Objekts dahinter, allerdings markiert es auch immer die Grenze, die nicht überwunden werden kann, wodurch das Objekt seinen musealen Status aufrechterhält.

65 Wo ich allgemein industriell zirkulierende Formen digitaler Reprisen als medienhistoriografisch wirksam untersuche, hat der Medienwissenschaftler Matthias Christen (2011) DVD-Editionen verschiedenen Paradigmen zugeordnet, nach denen diese historisches Wissen vermitteln: dem ökonomischen und dem philologischen Paradigma. Selbst 
Vor dem Hintergrund dieser Überlegungen soll ein letztes Beispiel gesondert vorgestellt werden, bei dem die Überlieferungsgeschichte von ideologischen Vorgaben und Eingriffen geprägt ist. Diese bestimmen nachhaltig die Bedingungen der heutigen Sichtbarkeit des Werks, welche ihrerseits einen historiografisch wirksamen medialen Erfahrungshorizont aufspannt: Erst in den 20ooer Jahren ist das Werk, ursprünglich Ende der 195oer Jahre in der DDR gedreht, vor allem über die Edition auf DVD der breiteren Öffentlichkeit zugänglich gemacht worden. Dabei ist der Film schon in seiner grundsätzlichen Anlage als Cluster aus zensierten Fragmenten zu sehen. So ergibt sich aus heutiger Sicht eine clusterhafte Mise en Relation, die auf die historische Praxis der Zensur in der DDR verweist beziehungsweise über die DVD und ihre Paratexte eine mediatisierte, implizite Vorstellung der Zensurprozesse vermittelt.

\subsection{Sinnlicher Eindruck von Zensurprozessen. Alternativen deutscher Geschichte. Die DVD DIE SchöNSTE (1957-1959/2003)}

„Verboten, verstümmelt und rekonstruiert - ein spannendes Kapitel deutscher Filmgeschichte." Mit diesem Schriftzug hat die Firma Icestorm 2003 ihre DVDEdition von DiE Schönste (1957-1959) versehen. ${ }^{66}$ Wie ein Label prangt er sowohl auf dem Cover als auch auf den begleitenden Heften der Edition, welche unter der Redaktion von Ralf Schenk entstanden sind. Damit wird das erste Mal für den Heimkinobereich, aber auch generell für ein breiteres Publikum das Werk zugänglich (s. Veröffentlichungsgeschichte unten). Das zitierte Label beschreibt in drei Schritten die Bedingungen für die aktuelle Edition auf DVD, welche das Werk bereits als historisches Cluster präsentiert. Denn diese bietet verschiedene Alternativen des Zugriffs an, die dem Zuschauer zur Auswahl stehen. Die DVD enthält als editiertes Hauptwerk zwei Fassungen: Sowohl die Ur- als auch die Zensurfassung mussten rekonstruiert werden, da der Film seit seiner Produktionszeit 1957-1959 in der DDR aufgrund ideologischer

Christen gesteht ein, dass diese Distinktion nicht mit absoluter Trennschärfe zu halten ist. Er kommt zu dem Schluss, dass filmrestauratorisches und quellenphilologisches Wissen zum Zwecke der Vermarktung eingesetzt werde - gerade im ökonomischen Paradigma. Ich wähle hier einen anderen Argumentationsverlauf, da ich aufgrund der eingeschränkten Möglichkeiten der Abgrenzung und generell angesichts der fehlenden Standards für Filmeditionen die These vertrete, dass gerade in der massenmedialen Zirkulation sich eine (medien)historiografische Wirksamkeit entfaltet und deshalb die Phänomene erinnerungskulturell in ebendiesem Horizont als bedeutsam zu begreifen sind.

66 Für den Hinweis auf den Fall von DiE Schönste danke ich Evelyn Strahm. 
Bedenken und sich verändernder politischer Konstellationen im Ostblock verboten war. Nach den ersten Dreharbeiten 1957 wurden immer wieder Umschnitte und Nachdrehs - auch mit Wechseln bei der Regie - vorgenommen, um das Projekt noch zu retten (vgl. hierzu als Übersicht die Zeitleiste im Booklet der DVD: 4-5). Der Film, ursprünglich ein Bühnenstück von Ilse Langner, hatte inhaltlich von vornherein mit dem Paradox zu kämpfen, eine kritische Haltung gegenüber dem bürgerlichen Westen und dem sogenannten ,Wirtschaftswunder' einnehmen und zugleich ein Unterhaltungsfilm für die Familie sein zu wollen. Im Mittelpunkt steht die Aushandlung von Schein und Sein. Es ist die Geschichte einer Wette zwischen zwei Jungen, welche Mutter die schönere sei. Zur Probe entwenden sie jeweils ein Schmuckstück, um zu beweisen, dass die Mütter auch ohne ihren Schmuck schön sind.

Diese narrative Grundsituation bedingte, dass man als Negativfolie den Luxus des westlichen Bürgertums zeigte und daraus gleichzeitig unterhaltende Schauwerte zog. Es ergab sich damit nach Rudolf Böhm, damaliger Chefdramaturg der DefA, im Laufe der Zensurmaßnahmen eine angespannte Erzählhaltung gegenüber dem eigentlich zu kritisierenden Gegenstand: „Wenn man die Brüchigkeit [der westdeutschen Gesellschaft, $\mathrm{FH}$ ] zeigen will, kann man sie nicht inmitten des Glanzes zeigen, der die Kritik überdeckt" (Böhm, zit. n. Schenk 2002c: 8). Zugleich, und dies erhöhte noch die Spannungen, sollte - so die ursprüngliche Planung in der Phase des ,Tauwetters' Mitte der 195oer Jahre - der Film in den Westen exportiert werden (deshalb waren auch zunächst ein Autor und Darsteller aus Westdeutschland möglich). Doch diese Stimmung schlug bereits Mitte 1957 schon wieder aufgrund weltpolitischer Ereignisse, etwa des Aufstands in Ungarn, um. Trotz weiterer Kürzungen und der Einfügung neuer Szenen sowie des Einschubs von an Brecht'sche Verfahren angelehnten didaktischen Zwischendialogen, die die Doppelmoral des ,Wirtschaftswunders' verdeutlichen sollten, kam der Film nie auf die Leinwand. Das Projekt verschwand in den Archiven. ${ }^{67}$ Selbst zur Wende 1989, als eine Kommission sich den Verbotsfilmen der DDR widmete - so Ralf Schenk in seiner Dokumentation -, sei der Film unbeachtet geblieben, weil er der Legende nach als relativ unpolitischer bürgerlicher Unterhaltungsfilm galt. Erst nach der Gründung der DEFA-Stiftung 1999 wurde das Werk im Zeitraum 2000-2002 zusammen mit dem Bundesarchiv-Filmarchiv unter den

67 Die Zensurabnahmen des Films dauerten bis Januar 1958. Hochrangige SED-Politiker und Kulturfunktionäre waren in den Prozess einbezogen. Die Meinungen waren weitgehend negativ: Eine Entlarvung des Wirtschaftswunders sei nicht zu erkennen gewesen, stattdessen wirke der Film ideologisch indifferent, wie ein ,Produkt der Traumfabrik' (Schenk 2002a: 8). 
veränderten institutionellen und politischen Bedingungen auf der Grundlage seiner unterschiedlichen Materialien und Manifestationen in einer ,Urfassung und ,Zensurfassung rekonstruiert. 2002 erfolgte die Uraufführung im Filmkunsthaus Babylon in Berlin (Schenk 2002a und b). ${ }^{68}$ Die Zensur- und Überlieferungsgeschichte der Versionen sind insofern eng an deutsche Ereignisgeschichte geknüpft, die jeweils ideologische Normierungen des Filmmaterials zur Folge hatte.

Unter der Redaktion von Ralf Schenk wurde Anfang der 2oooer Jahre das Konvolut von überlieferten Materialien zu einer DVD editiert: So enthält die DVD die zwei rekonstruierten Fassungen sowie rahmende, ergänzende, kommentierende und kontextualisierende Zeugnisse und Dokumente, die sich über schriftliche wie audiovisuelle Formen vermitteln.

Die auf diese Weise multimedial vermittelten historischen Prozesse und Praktiken bilden die wichtigsten Bezüge für die Herstellung des Verhältnisses die Mise en Relation - der Filmfassungen zueinander. Die beiden Fassungen werden im Menü der ersten DVD wie folgt kopräsent angezeigt - unter der Maßgabe einer Auteur-Kategorisierung mit Blick auf den jeweiligen Regisseur; dies vor dem Hintergrund einer Menügestaltung, die in das Thema des Films einführt: Die Protagonistin Frau Berndorf, als Unternehmergattin und Mutter Projektionsfläche für die titelgebende Schönheit, die allerdings an den Besitz eines Perlenkolliers gebunden ist, steht im Mittelpunkt. Das glänzende Perlenkollier stellt auch gleichzeitig das Icon zur Auswahl des jeweiligen Schriftzugs der Fassung dar. Auszuwählen sind (Abb. 7.19):

- die Urfassung Ernesto Remani,

- die Zensurfassung Walter Beck,

Man kann also beide Fassungen linear in ihrer Rekonstruktion von Ralf Schenk als integrale (eigenständige) Filme anschauen. Gleichzeitig bekommt man allerdings unter dem Menüpunkt der Zensurfassung nun die Variationen noch einmal in Einzelkapiteln offeriert - also als Fragmente ohne den narrativen Zusammenhang der Story (nur angedeutet über ,Kapiteltitel'). Es ist strukturell zunächst eine vor allem auf den Regisseur bezogene Aufschlüsselung der Unterschiede. Der Nutzer kann also wie folgt auf die narrativen Fragmente zugreifen (Abb. 7.20):

68 „Zum ersten und einzigen Mal in der DEFA-Geschichte wurden an einem Abend zwei komplette Fassungen ein- und desselben Films gezeigt. Die überlieferten Materialien hatten sowohl die Rekonstruktion der ersten, dem Drehbuch entsprechenden Urfassung von 1957 als auch der stark gekürzten, durch neue Szenen erweiterten Zweitfassung gestattet" (Schenk 2002c: 9). 


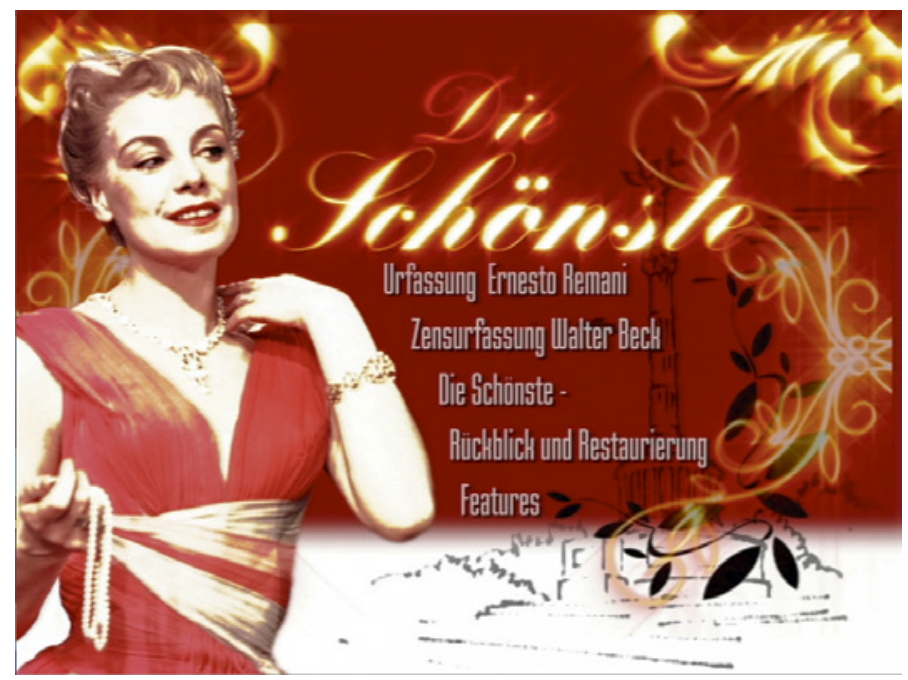

Abb. 7.19

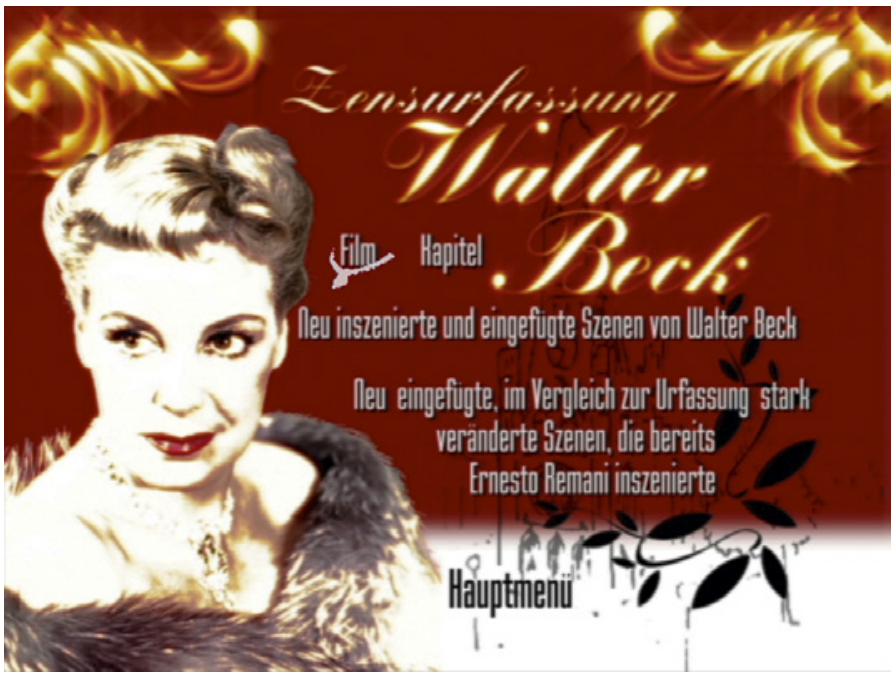

Abb. 7.20

- neu inszenierte und eingefügte Szenen von Walter Beck, die dann nummeriert in einer Liste einzeln anwählbar sind. Die Szenen haben jeweils kurze, inhaltsangebende Titel;

- bereits von Ernesto Remani nachinszenierte/gedrehte Szenen.

Diese Überlieferungsgeschichte wird auf mehreren Ebenen ergänzend narrativiert und unter anderem in einen Kontext gesetzt:

- durch das ausführliche Booklet, das wiederum über eine Zeitleiste eine chronologisch geordnete Übersicht schafft unter dem Titel Chronik eines 
Filmverbots sowie über einen Fließtext, der die Geschichte des Films erzählt (Schenk 2002a: 6-9);

- durch den filmisch erzählenden Paratext Die Schönste - RÜCKblick und Restaurierung einer filmhistorischen Dokumentation, in der der verantwortliche Redakteur meist als Talking Head die Zusammenhänge noch einmal in Vortragsform performativ vermittelt. Auch Zeitzeugen der Produktion (Szenenbildner, Aufnahmeleiterin, zweiter Autor für Änderungen, Schauspielerin) kommen zu Wort und stellen über ihre Erinnerungen eine Verbindung zwischen den Filmbildern und der Gegenwart her. Insbesondere die Schauspielerin Gisela May betont das Unbehagen, sich selbst als jugendliche Schauspielerin noch einmal auf der Leinwand zu sehen - in Filmbildern, die sie vorher nie hat sehen können. Ihre Person, Biografie und Lebensdauer schaffen die Verbindung zu dem Film, der nun erst, das Licht der Welt ${ }^{\text {- }}$ so die Implikation der Dramaturgie der Dokumentation - erblickt. Die visuelle Inszenierung unterstützt die doppelte neue Sichtweise des Films, die sich metaphorisch hier andeutet: Talking Heads der Zeitzeugen und Stills aus dem Spielfilm werden in einer fenster- oder leinwandähnlichen Mise-en-Abyme-Struktur zusammen im Bild gezeigt.

- Auf der zweiten DVD finden sich noch diverse nichtfilmische Materialien, etwa Informationen zu Produktionsbeteiligten, aber auch weitere Archivmaterialien wie Fotos, Zulassungsprotokolle, das Drehbuch oder Partituren. Im begleitenden Booklet steht unter der Auflistung der ganzen Zusatzmaterialien „Viele Dokumente zum Ausdrucken und Archivieren!". Auf diese Weise wird zum einen der Gestus des Entdeckens, des Recherchierens beim Nutzer angeregt, zum anderen setzt sich auch der Aneignungsgestus des Mediums der DVD fort: Es geht nicht mehr nur darum, die Box oder den Film zu besitzen, ${ }^{69}$ sondern zugleich über die Praxis des Sammelns auch historische Zusammenhänge selbst zu entdecken und konstruieren zu können. ${ }^{70}$ Die Überlieferungsgeschichte als Cluster wird zum Mehrwert des Films. Die DVD wird in ihrer medialen Erfahrung zum Medium der Vermittlung der Rekonstruktion, die sich nicht nur philologisch affirmativ verhält, sondern in der Intermedialität, in dem Dazwischen der Montage der Materialien, Projektionen des Nutzers auf die historische Dimension des editierten Werkes zulässt. Dies überträgt sich auf das Erlebnis der jeweiligen Filmfassung,

69 Zur Fetischisierung des Archivs - auch in seinem Sammlungsgestus - vgl. H. Böhme (2012) in $\rightarrow$ Kap. 4.

70 Wieder sei an dieser Stelle auf die Zusammenhänge von Sammlerpraktiken und Diskursen um Cinephilie verwiesen; vgl. hierzu grundsätzlich Valck/Hagener (2005). 
wo anhand der ästhetischen Erfahrung immer das Moment der möglichen Alternativen kopräsent ist.

Dass ein vermeintlich ,bürgerlicher Unterhaltungsfilm` solche historischen Transformationsprozesse durchlaufen hat, lenkt den Blick auch auf die inhärenten Ambivalenzen der filmischen Bilder selbst. In der Mise en Scène setzt sich das Thema des (falschen) Scheins vor allem in der Darstellung der Lebensverhältnisse der Familie des Geschäftsmannes um. Die Räume in der ausladenden Villa haben eine symbolisch aufgeladene Ausstattung, die fast an die Hollywood-Melodramen von Douglas Sirk erinnert, die Kostüme schillern in Agfacolor (Abb. 7.21).

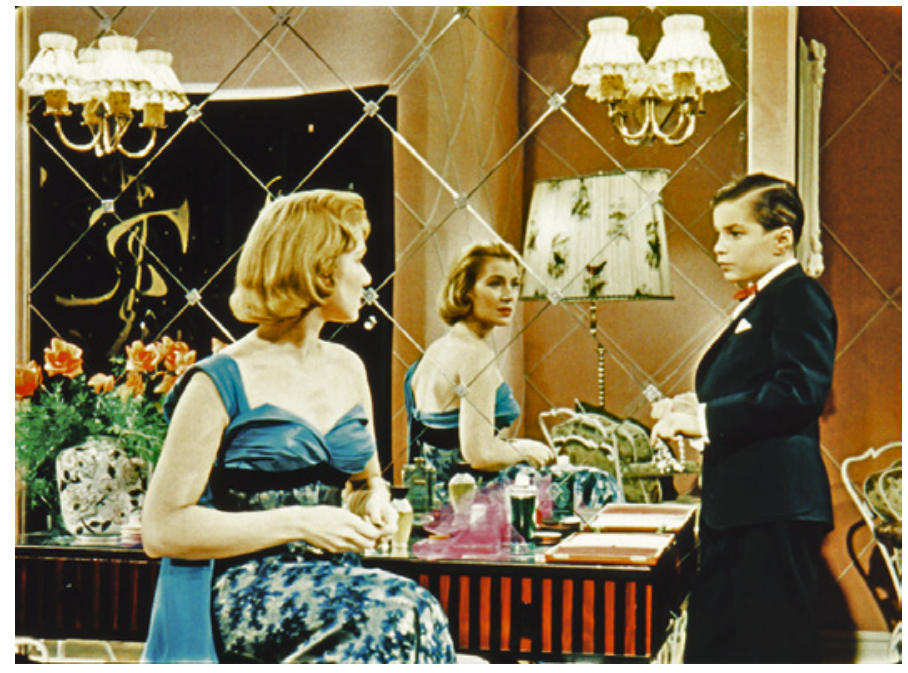

Abb. 7.21

Der Glanz und Schein der Oberflächen überträgt sich in den Aufbau der Räumlichkeiten: Oft ist nicht klar zu erkennen, wo in den Boudoirs die Türen in den Wänden und Spiegeln der üppigen Wanddekorationen versteckt sind. Der Reichtum, der eigentlich entlarvt werden soll, verleiht gerade in solchen Bildern dem Film seinen Schauwert und unterläuft so im Sinnlichen die eigentliche antikapitalistische Botschaft. Diese sollte entsprechend in den Nachdrehs von Walter Beck, basierend auf extra angefertigten Texten vom Brecht-Schüler Heinz Kahlau, mit einer , agitatorischen Kommentierung über eine Rahmenhandlung zweier Maler und eines Bänkelsängers (gespielt von dem jungen Manfred Krug) deutlicher hervorgebracht werden - der ganze Schein ist Fassade, die letztendlich immer zerbröckeln wird; kulminierend im hinzugefügten letzten Bild des Films: Die Maler überstreichen das Blickfeld der Kamera (Abb. 7.22). 
Abb. 7.22

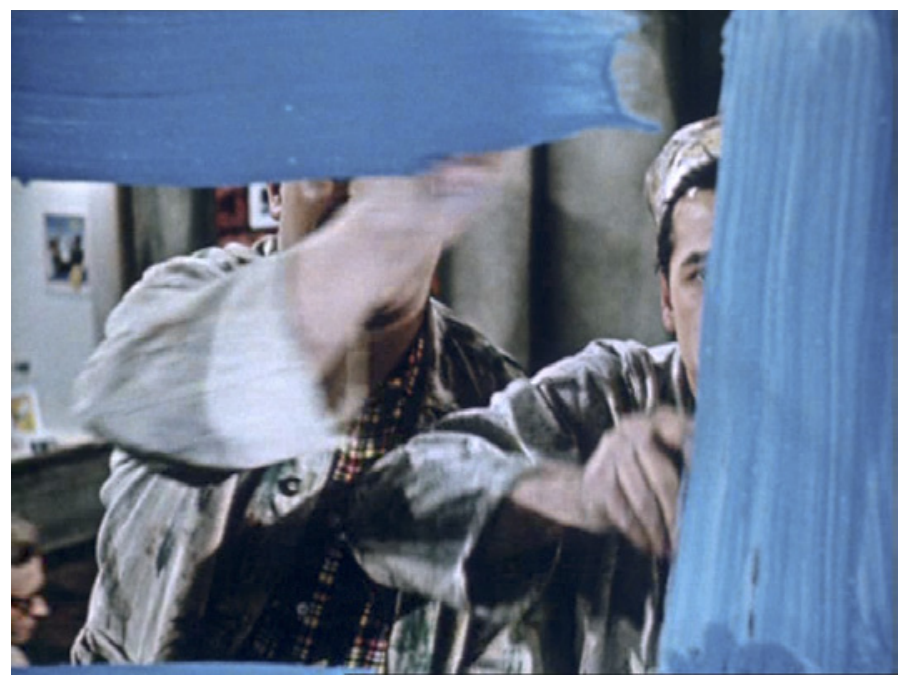

Man könnte dies wiederum metaphorisch wenden und angesichts der ständigen Veränderung des Films die These formulieren, dass in den historischen Praktiken versucht wurde, die Schauwerte des Filmbildes palimpsestartig zu überschreiben - was nun die DVD räumlich und zeitlich aufschlüsselt und auffächert. Für meine Argumentation bedeutet dies, dass der Prozess der Rekonstruktion und der Überschreibung sich wirkungsästhetisch in der sinnlichen Wahrnehmung beim Nutzer als (Nach-)Vollzugsinstanz vollzieht.

Entsprechend verhalten sich dann auch die beiden unterschiedlichen Enden: Entweder wird der Schein aufrechterhalten. Die bourgeoise Unternehmerfamilie bleibt zusammen, Lug und Betrug sind vergeben. Oder aber Mutter und Sohn verlassen gemeinsam den betrügerischen Vater und Ehemann.

In Anlehnung an vorherige Beispiele könnte man in dem Dispositiv dieser DVD mit ihren explizit historischen Referenzen die Struktur einer historiografischen Multiple-draft- oder auch Forking-path-Narration ausmachen; ${ }^{71}$ weder die eine noch die andere ist falsch. Oder mit Hartmann (2012) gesprochen: Der Geltungsanspruch der Geschichte ist ausgesetzt, wird möglicherweise überlagert von der subjektiven Beurteilung des Geschehens.

71 Dieser Ansatz versteht sich als Versuch, im Anschluss an Hayden White (2008) poetologische und narrative Strategien der (mediatisierten) Historiografie zu untersuchen; im vorliegenden Fall sind es Modelle aus filmnarratologischer Perspektive. Des Weiteren verweist ein solches Muster der Geschichtsschreibung respektive des Geschichtserlebens auf medienarchäologische Modelle, wie sie mit Elsaesser (u. a. 2004) in $\rightarrow$ Kap. 3 beschrieben worden sind. 
Erneut profiliert meine Perspektive die medienhistoriografische Wirksamkeit im Erlebnismodus des Vergleichs über die spezifische Mise en Relation, wobei Thema, Struktur und Ästhetik des editierten Spielfilms wiederum eine wichtige Rolle einnehmen: Der historisierende Nachvollzug von möglichen Zusammenhängen (im Sinne einer historisierenden Lektüre in Anlehnung an Odin) geschieht in Verbindung mit dem Modus der Unterhaltung im Glanz der ambivalenten, ästhetisch exzessiven Bilder. So wird Geschichte ohne festen Referenten sinnlich-medial als Praxis von filmisch-atmosphärischen Differenzen erfahrbar. Der Nutzer kann über den imaginären Vergleich, die mögliche Varianz der rezipierten Fassung die historische Zensurpraxis ex negativo aufgrund des offenen Ensembles in der Konfiguration der DVD erfahren - auch im Modus der Re-Montage und des Fragmentarischen. Letzteres gilt insbesondere für die einzeln editierten Sequenzen. Die historische Praxis wird so auch für die (Markt-)Öffentlichkeit individuell interpretierbar - je nach persönlicher Wahl und entsprechender Mise en Relation; dies allerdings immer im Modus der unterhaltsamen Spurensuche mit überbordenden Schauwerten, wodurch das Beispiel ein „spannendes Kapitel“ der deutschen Filmgeschichte bleiben wird. Die historische Spurensuche verwebt sich in diesem Fall anschaulich und nachdrücklich mit der Wahrnehmung der filmischen Fiktion, die auf den melodramatischen Exzess baut und damit einen sinnlichen Überschuss produziert (vgl. $\rightarrow$ Kap. 6). Die DVD auratisiert das historische Cluster, der Nutzer betreibt - zugespitzt zusammengefasst als Projektionsinstanz die Rekonstruktion von historischen Zusammenhängen im Erfahrungsmodus des filmästhetischen Exzesses. Eine pikante Pointe ist nicht zu übersehen: Der Exzess der Bilder sollte ursprünglich eigentlich gesellschaftliche Verblendungszusammenhänge ausstellen (woran er schon zu seiner Produktionszeit scheiterte). Heute trägt der Glanz der Bilder nicht nur zur faszinativen Wirkung und zum kulturellen, erinnerungswürdigen Status des Films bei. Darüber hinaus vermittelt der schöne Schein der Bilder implizit ausgerechnet historische sozialistische Denk- und Wahrnehmungsweisen, die sich in der Erfahrung der Mise en Relation der Versionen und/oder der jeweiligen Fragmente beim nutzenden Zuschauer einstellen können.

\subsection{Mise en Relation der Mythen I. Die Nibelungen (1924) und die Überlieferungsgeschichte in heutiger audiovisueller Perspektive}

Die Auratisierung von Filmen als filmhistorische Cluster in digitalen Dispositiven soll in der Folge in einer weiteren Analyse eines DVD-Paratextes, einer filmhistorischen Dokumentation, erläutert werden, die den editierten 
Film als sogenanntes Bonusmaterial rahmt und kommentiert. Die an dieser Stelle untersuchte Ausprägung der Mise en Relation liegt vor allem in filmischen Konfigurations- und Vermittlungsformen: Die Relativierung von historischer Wahrnehmung wird audiovisuell verhandelt und für den Zuschauer in spezifischer Weise sinnlich erfahrbar gemacht. In diesem Fall soll - im Zeichen von Formen der Mise en Relation - das Moment der Legierung von filmischer Fiktion und Non-Fiction-Material ${ }^{72}$ als ästhetische Ausprägung einer historiografisch wirksamen Relativierung von Geschichtsbildmodellierungen hervorgehoben werden. Das besprochene Exempel ist die Dokumentation DAS ErbE Der Nibelungen, die der Eureka-DVD-Edition 2012 von Fritz Langs Die NibELUNGEN (1924) beigelegt ist.

Die Dokumentation verhandelt Versionenhaftigkeit als filmhistorische Konfiguration auf mehreren Ebenen: Zum einen zeigt sie das Problem verschiedener Fassungen als Referenzen im Restaurierungsprozess auf. Zum anderen werden verschiedene Fassungen als Effekt von - in diesem Falle ideologischen Normierungen, die Überlieferungsvarianten zur Folge haben, illustriert. Die dynamische zeitliche Dimension einer solchen Überlieferungsgeschichte, in der historische Umstände das Filmwerk inhaltlich, formal- wie rezeptionsästhetisch prägen, steht in einer Spannung zu dem Thema des Films und dem damit auch verwobenen, sich mehrfach re-konfigurierenden Status des Werks als überzeitlichem Mythos.

Die tatsächliche Fassungsgeschichte zeitigt deutliche Folgen für die vorliegende DVD-Edition und den Film, der über sie zugänglich wird: Zum einen gilt dies für den materialästhetischen Restaurierungsprozess in Fragen der Bildgestaltung und -qualität. Zum anderen haben die Überlieferungsund Rezeptionsgeschichte Einfluss auf die Story, wenn es um Aspekte der dramaturgischen Rekonstruktion einer möglichst kompletten Version geht. Dass es dann dazu eine filmische Ab- und Aushandlung im Format einer Dokumentation gibt, die als Bonusmaterial eines digitalen Dispositivs funktioniert, macht den Fall als jüngere Aktualisierungspraxis auf mehreren Ebenen interessant.

Wie bereits einleitend erwähnt, stellen inhaltlich die beiden Teile von Die Nibelungen ein eindrückliches Beispiel für Zeitkonzeptionen und Geschichtsbilder aufgrund der inhaltlichen Thematik dar. Der Stoff ist ein archaischer deutscher Mythos, der schon, bevor er verfilmt wurde, auf viele verschiedene Weisen in unterschiedlichen Medien und Kunstformen aufgegriffen und erzählt wurde (u. a. H.-B. Heller 1991). Im Anschluss daran wurde

72 Vgl. zu diesem Verfahren ausführlich $\rightarrow$ Kap. 6. Allerdings erfolgten dort die Analysen mit Fokus auf die Bedeutung des Imaginären und der Imagination. 
der Film von Fritz Lang selbst ein Mythos - auch unter den Vorzeichen einer (retrospektiven) Nobilitierung durch eine Auteur-geprägte Perspektive mit Blick auf den Regisseur Lang.

Die Dokumentation DAS Erbe Der Nibelungen (Altendorf/Wilkening, 2011) beginnt mit der Frage, was von dem Filmset aus den 1920er Jahren überlebt hat, als Lang entschied, alle Dekorationen des Films zu zerstören. Dies findet in der Dokumentation sein ikonisches Bild, in dem der Brand der Kulisse der Etzelburg auf dem Filmgelände in Babelsberg gezeigt wird (das gleichsam Eingang in den zweiten Teil, KRIEM HILDS RACHE, und somit in die filmische Fiktion fand, Abb. 7.23).

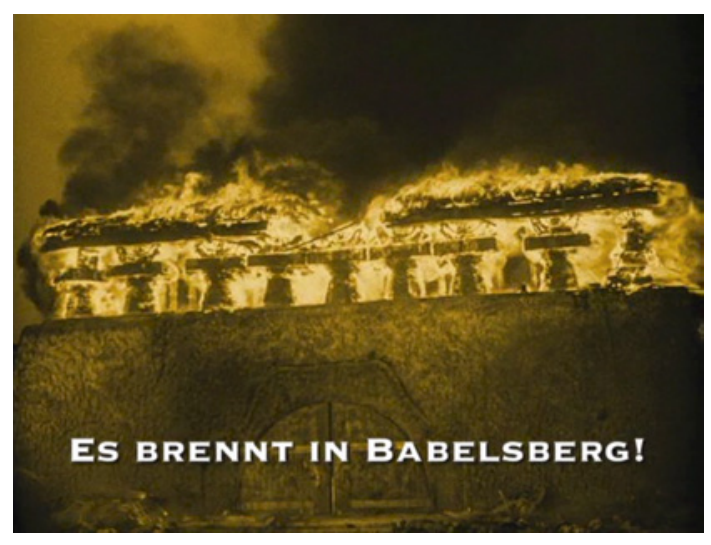

Abb. 7.23

Die männliche Voice-Over der Dokumentation fragt anhand dieser Bilder, was eigentlich noch an sichtbaren Spuren des Films in Babelsberg übrig geblieben sei. Das hier etablierte diskursive Leitmotiv der Frage nach sichtbaren Formen und Monumenten der Erinnerung - hier am konkreten Beispiel der Gebäudekulissen - wird visuell und rhetorisch immer wieder von der Dokumentation aufgegriffen. Dies gilt insbesondere eben auch für die komplizierte Frage, wie Bilder eines Filmes überleben, wie die Bilder unter wechselnden historischen Bedingungen zirkulieren und ihre Bedeutung verändern.

Zunächst wird das Problem der Überlieferung von der Dokumentation in einen restaurationspraktischen Kontext gesetzt: Es werden die besonderen Umstände der Filmproduktion von Die Nibelungen erklärt - und dies wird bedeutsam für die Frage nach der Referenz und nach der Bestimmung eines Originals, was zentral für jeden Restaurierungsprozess ist. Zur Erinnerung: Jede Restaurierung, so jüngere pragmatische Ansätze zur Theorie der Filmrestaurierung, kreiert ein eigenes Dispositiv (Fossati 2009, Wilkening 
2014). Damit bringt jede Restaurierung die Voraussetzungen, unter denen sie ihr Original definiert, selbst hervor - über die jeweiligen institutionellen, technologischen, ästhetischen und ethischen Entscheidungsprozesse als Bedingungen der Referenz und des Restaurierungsresultats. Die NibeLungeN sind hier ein besonderer Fall: Der Film wurde mit mehreren Kameras gedreht, wodurch es somit mehrere ,Original'kameranegative gibt, die sich leicht im Bildwinkel unterscheiden.

In der Dokumentation wird dies vergleichend über einen Split Screen mit Fragmenten aus dem Film illustriert (Abb. 7.24-7.26).

Abb. 7.24
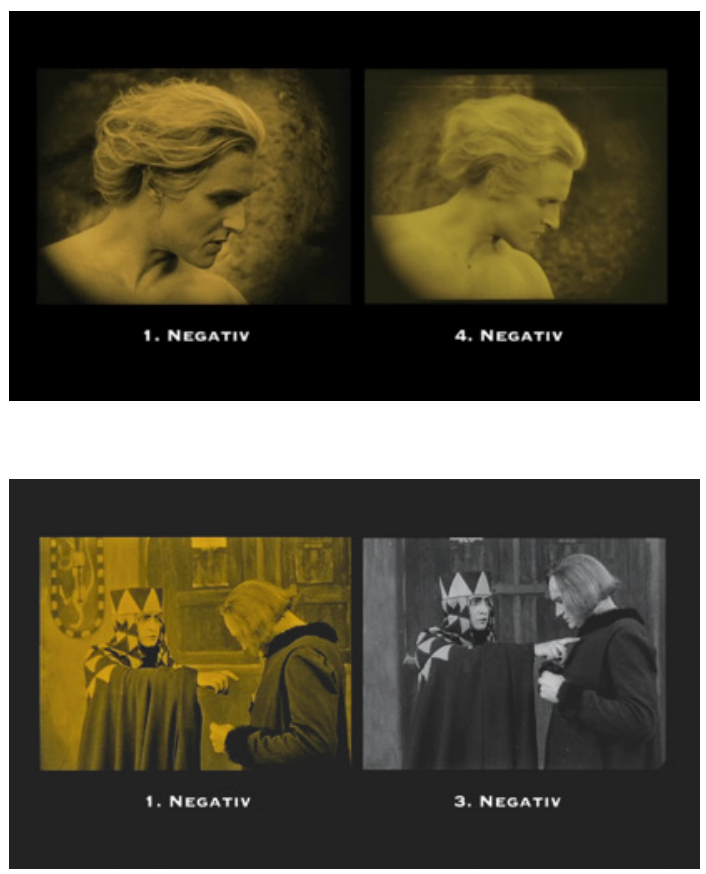

Abb. 7.25

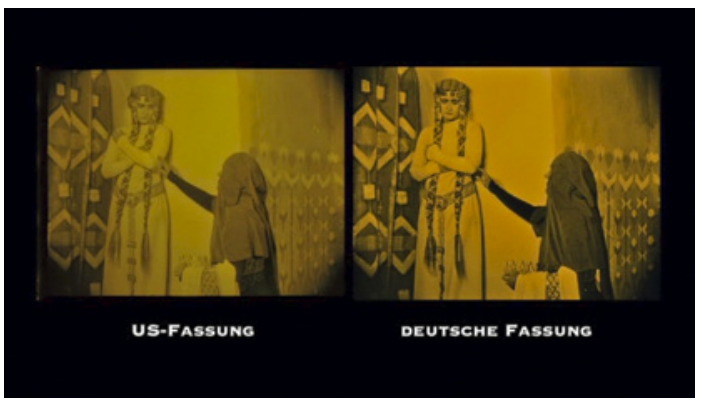

Abb. 7.26 
Wie schon an anderer Stelle erwähnt (vgl. $\rightarrow$ Kap. 4 und $\rightarrow$ Kap. 6), nehmen Split-Screen-Konfigurationen eine wichtige Funktion in filmhistorischen Dokumentationen ein - insbesondere in der Vermittlung von Restaurierungspraktiken. Sie sind ein wichtiges wirkungsästhetisches Mittel der Mise en Relation (vgl. auch unten die weitere Konzeptualisierung des Split Screens als Wahrnehmungsdispositiv). Eine Split-Screen-Konfiguration macht in einem solchen filmischen Kontext die Varianz von materialästhetischen Merkmalen und Bildqualitäten für den Zuschauer - gemäß meinem Sprachgebrauch - sinnlich nachvollziehbar: Auf diese Weise, wie etwa auch schon im Zusammenhang der Re-Editionen von Peter Pan oder Le Voyage dans LA Lune erläutert (vgl. $\rightarrow$ Kap. 4 und $\rightarrow$ Kap. 6), wird der Zuschauer zur performativen (Nach-) Vollzugsinstanz nicht nur des Unterschieds, sondern - je nach Kontext - auch des qualitativen Sprungs (einer Verbesserung). Über den Modus des Vergleichs wird der Zuschauer angehalten, die visuelle Evidenz nachzuverfolgen und damit zu affirmieren, dass eine Veränderung stattgefunden hat. Ähnliche Verfahren kommen zur Anwendung, wenn verschiedene Fassungen inhaltlich verglichen werden: Welche Bilder sind in welcher Version vorhanden? Auch dies wird über Bildmontagen in Form von Split-Screen-Anordnungen dem Zuschauer anschaulich und zugleich erfahrbar gemacht.

Im Falle der Einschätzung der überlieferten filmischen Quellmaterialien von Die Nibelungen, die hier in der Dokumentation in verschiedenen SplitScreen-Konfigurationen einer Mise en Relation unterzogen werden, geht es vornehmlich darum, die Schwierigkeiten der Bestimmung eines Originals und damit auch das Problem der Versionenhaftigkeit von Filmen zu vermitteln, was letztendlich den Zuschauer für die notwendigen Entscheidungsprozesse in der Filmrestaurierung sensibilisiert.

Im Fortgang der Dokumentation verlegt sich das Verfahren der Mise en Relation und der Kontextualisierung mit Blick auf die weitere Überlieferungsgeschichte von Die Nibelungen allerdings zunehmend auf das Prinzip des linearen Montageflusses unterschiedlicher Bildregister, was in Kombination mit den Inhalten der Bilder spezifische Folgen für das vermittelte Geschichtsbild zeitigt. Die Rezeption des Films wurde - so erzählt es die Dokumentation - in den 193oer Jahren äußerst ideologisch und damit politisch aufgeladen. ${ }^{73}$ Goebbels, so zitiert die Dokumentation, bezeichnete Die Nibelungen zu dieser Zeit als einen Schicksalsfilm mit Vorbildfunktion („das deutsche

73 Der Film hatte schon zu dieser Zeit - neben anderen Veränderungen - eine technologische Normierung durchlaufen: Ab Mai 1933 gab es auch eine Tonfilmfassung des ersten Teils, der in den Kinos in Deutschland lief. 
Schicksal schlechthin“), der die Zeit überdauere: „Wie alt ist dieser Film schon, und doch, wie modern". ${ }^{74}$

Diese Tendenzen, den Film ideologisch und erinnerungspolitisch als Mythos zu funktionalisieren, werden von der Dokumentation durch spezifische ästhetische Verfahren aufgegriffen und vermittelt. Die Aneignung des Films durch den Nationalsozialismus wird über eine Montage umgesetzt, die zeitgenössische Non-Fiction-Archivbilder mit Fragmenten aus den Lang'schen NibeLUnGEN-Spielfilmen verschmilzt. Dies wird auditiv auf zwei Ebenen ergänzt, indem zum einen die Voice-Over bedeutungsgebende Verbindungen schafft. Zum anderen wird O-Ton etwa von Goebbels über die Bilderfolgen gelegt. Somit liegt ein argumentativer und ästhetischer Schwerpunkt der Dokumentation auf der Demonstration der Legierung von Realität und filmischer Fiktion in Hinblick auf die Etablierung eines kollektiven, kultischen Gedächtnisses, das die Massen ergreift. Die Voice-Over der Dokumentation hebt in diesem Sinne die grundsätzliche Bedeutung eines Spielfilms für die Geschichtsbildung und Identität der nationalsozialistischen Ideologie hervor: „Tonscherben taugen nicht als Zeichen des heldischen Aufbruchs, [...] das NS-Kino bedient sich des Nibelungen-Films. Seine Bilder werden vereinnahmt, kopiert und übersteigert. Mit Pomp werden Tote ausgegraben, umgebettet und zu Helden erklärt." In der Aneignung der Bilder des Spielfilms wird ein mehrdeutiger ritueller Erinnerungskult aufgebaut, der sich aus einem das Individuum übersteigenden Zeitbegriff speist - so die Erklärungen des Kommentars.

Die Dokumentation greift dies formal auf, indem zu diesen Worten Bilder aus Die Nibelun gen montiert werden mit Non-Fiction-Material, das nationalsozialistische Massenaufmärsche und öffentlich inszenierte Beerdigungen zeigt (Abb. 7.27).

Auf diese folgen wiederum offensichtlich inszenierte Szenen von germanisch-archaischen Beerdigungsritualen aus dem Film EwIGER WALD (Springer/Sonjevski-Jamrowski, 1936). Die Referenz auf diesen Film wird kenntlich gemacht durch die schriftliche Einblendung seines Titels (Abb. 7.287.29). Dazu hört man den diegetischen Ton weihevoller Chorgesänge. Kulturhistorisch ist der hier einmontierte, heute kaum mehr bekannte Film EWIGE R WALD einzuordnen als „(g)roßangelegter Versuch, im Sinne der ,Blut und Boden-Ideologie der Nationalsozialisten die Geschichte des deutschen Volkes als Geschichte des deutschen Waldes zu erzählen, hergestellt im

74 Zu dem historischen wie in jüngerer Zeit geführten Diskurs um den NibELUNGEN-Film im Zeichen des Zusammenhangs von ,Mythos' und aktuellem ,Zeitbild“ (Fritz Lang) vgl. auch mit Bezug auf Langs explizite Selbstkommentierungen - H.-B. Heller (2003). 


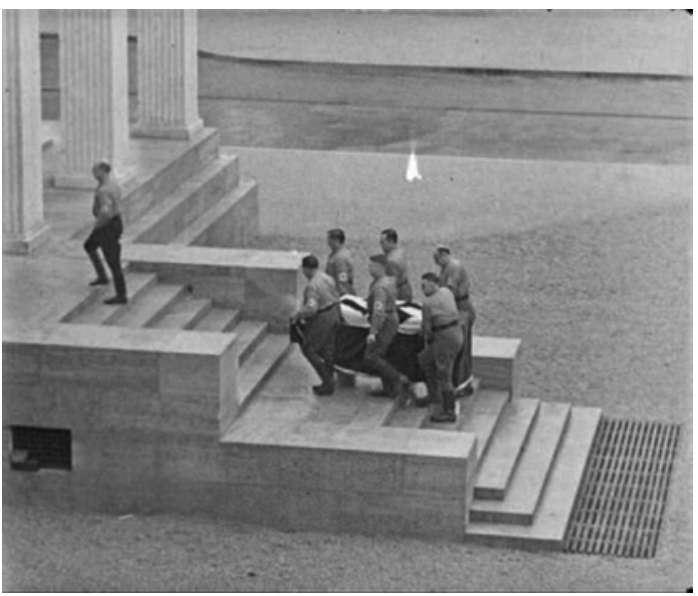

Abb. 7.27

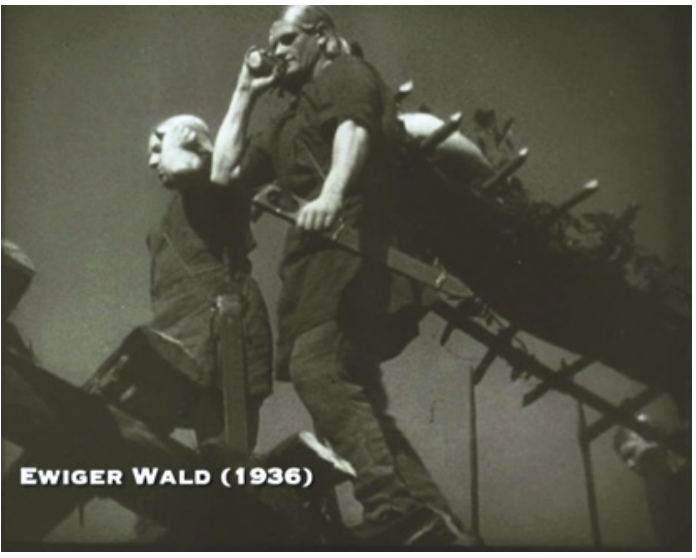

Abb. 7.28

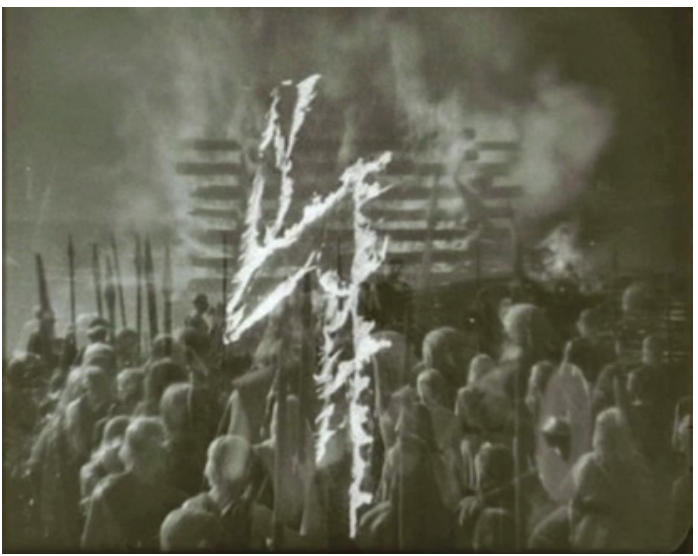

Abb. 7.29 
Auftrag der NS-Kulturgemeinde“ (Filmportal.de zu EWIGER WALD). EWIGER WALD arbeitete selbst mit der Vermischung verschiedener Bildregister und propagierte damit ein auch außermedial wirksames Geschichtsbild im Sinne der Naziideologie: „In einer Mischung aus Dokumentar- und Spielszenen spannt der Film seine Handlung von der Bronzezeit bis in die Gegenwart" (Filmportal.de zu EWIGER WALD).

Diese mythische Zeitideologie wird - so die Argumentation der Dokumentation der DVD aus dem Jahr 2011 - immer mehr mit der Fiktion des Mythos verwoben: Siegfrieds Schwert werde immer weiter geschmiedet. Daran schließt eine Montage von Filmausschnitten unterschiedlicher Provenienz an, die alle ikonografisch auf Siegfrieds Waffe rekurrieren (Abb. 7.30-7.33) - bis hin zu dem Zitat eines „Kurz-Dokumentarfilms“ (Filmportal.de), dessen Titel auch den wirkungsästhetischen Zweck der Aneignung der filmischen Bilder beschreibt: Deutsche Vergangenheit Wird Lebendig (Kifo/Hellmut Bousset, 1936).
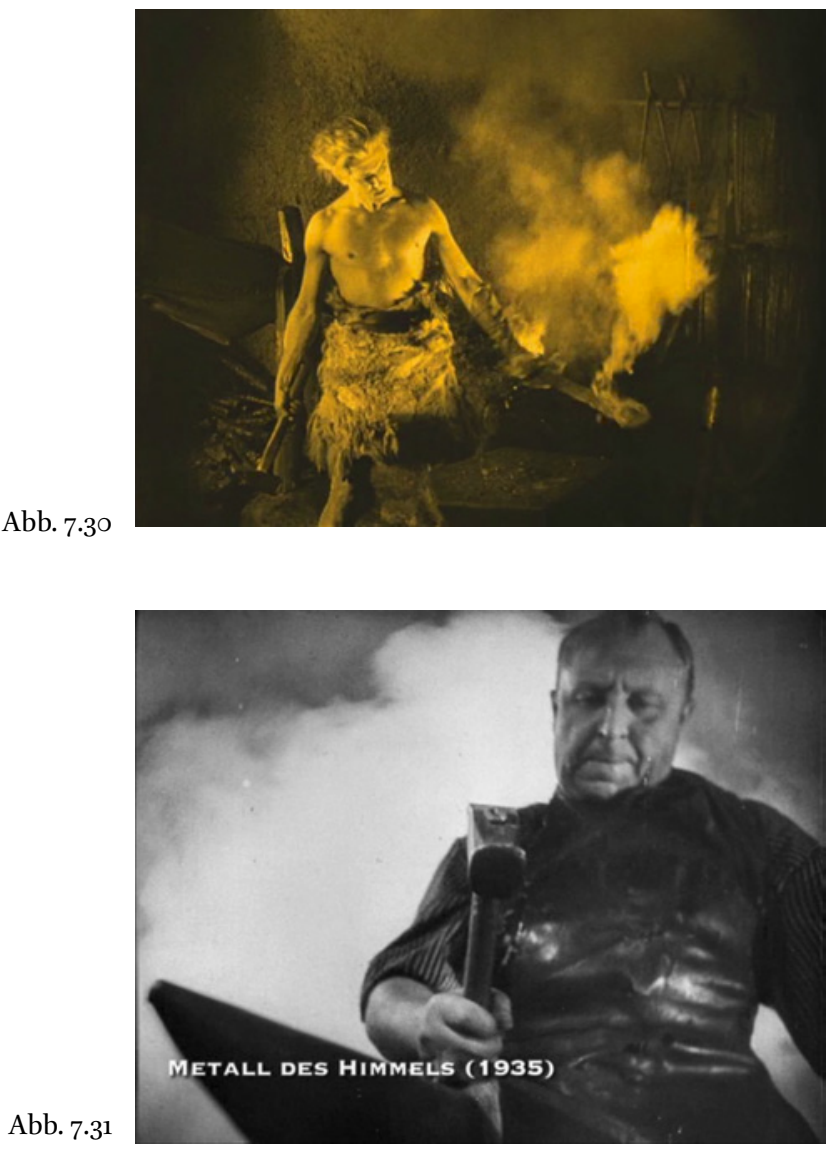


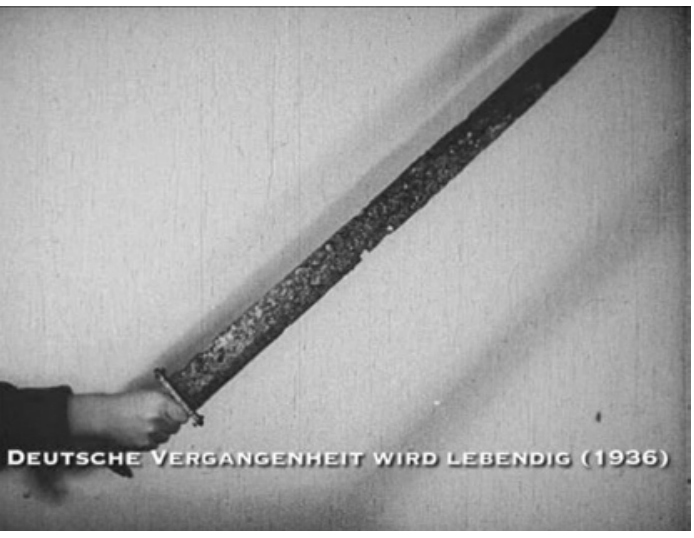

Abb. $7 \cdot 32$

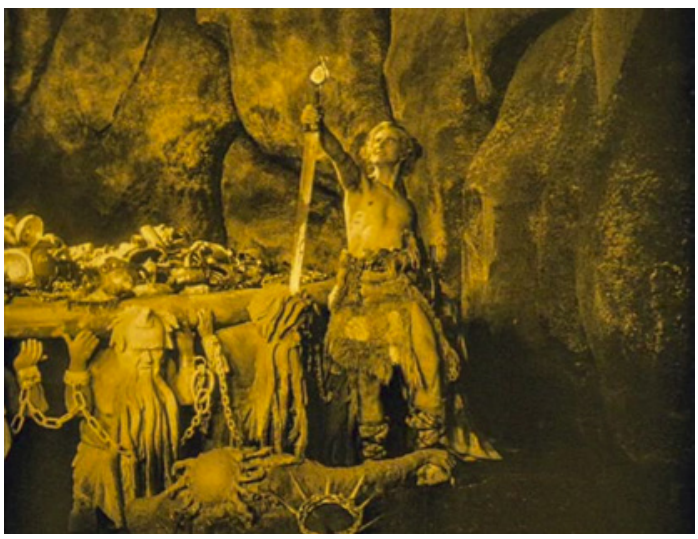

Die DVD-Dokumentation zeigt über die Montage verschiedener Materialien, wie der Nibelungen-Film, insbesondere der erste Teil, als eine steinbruchartige Quelle für symbolische Bilder verwendet wurde, um ein die Zeit und das Individuum überdauerndes mythisches Geschichtsbild und Selbstverständnis zu etablieren. Angesichts eines solchen Montageflusses wird der Zuschauer ständig mit der Notwendigkeit konfrontiert, zwischen den unterschiedlichen Bildregistern zu unterscheiden beziehungsweise sie einer entsprechenden Lektüreform zuzuordnen. Meine These ist allerdings, dass - aufgrund der pasticheartigen Montageform im Zusammenspiel mit der präsentisch wirkenden Eindrücklichkeit und ästhetischen Dichte bestimmter Bilder - in der Dauer des Montageflusses apriorische Distinktionsgrenzen zwischen den Lektüreformen verschwimmen. Der Zuschauer muss für sich ständig selbst die Bilder vergleichend bewerten, er muss zwischen erkennbaren Implikationen der zitierten Ausgangsfilme, ideologisch überformten Aneignungsformen und 
kritisch-diskursiver Narration aus heutiger Sicht mit restaurationspraktischem Interesse komparatistisch und situativ abwägen.

Für die Fassungsgeschichte des Films haben die nationalsozialistischen Aneignungsformen zur Folge, so argumentiert die Dokumentation von 2011 weiter, dass der Nibelungen-Film von Fritz Lang zu einem gefledderten Torso reduziert wurde, der hochgradig ideologisch kontaminiert war. Dies, so die Logik und Narration der Dokumentation, sei einer der Gründe für die Existenz verschieden überlieferter Versionen von Langs Nibelungen, für deren unterschiedliche, zumal kontrovers beurteilte Wertigkeit und damit nicht zuletzt für die insgesamt problematische Ausgangssituation eines jeden Restaurierungsprojekts dieses Werkes:

Der NS-Kulturfilm stellt Die Nibelungen als ausgeweideten Torso ins Regal der Filmgeschichte [...]. Der Nibelungen-Film ist geplündert und hinter den nationalsozialistischen Zitaten und Plagiaten verschwunden. Was für lange Zeit geblieben ist, sind ideologisch belastete Trümmer, die nicht mehr zusammenzusetzen sind (VO der Dokumentation).

Um den Weg in die Gegenwart der Restaurierung zu finden, fragt die Dokumentation in der Folge, was unter diesen Vorzeichen von dem Film nach dem Krieg übrig geblieben sei. Die Antwort überrascht, da sich der Mythos des Films in den 195oern in einer neuen performativen Aktualisierungsform wieder zeigte. Die DVD-Dokumentation führt vor, wie in einer VaudevilleShow (1958) der Film in Kompilation, in Form von kommentierten Versatzstücken zur Aufführung kam: Unter dem Titel ,DAS GAB'S NUR EINMAL ... Hans Albers präsentiert die Schönsten Filme unseres lebens ... führt der berühmte Schauspieler als Moderator durch ein Showprogramm mit Filmzitaten. Hans Albers, selbst noch gefeierter Darsteller unter den Nationalsozialisten, wurde nun zum (Kino-)Erzähler des Filmmythos DiE Nibelungen.

Ein Sprung zum Heimkinomarkt: Die DVD-Dokumentation erklärt weiterhin, dass die Filme unter den oben beschriebenen Vorzeichen in verschiedenen, oft gekürzten Fassungen in Umlauf gekommen sind, was sich auf dem Heimkinomarkt über den VHS-Vertrieb fortgesetzt habe. Mit Bezug auf die Aussagen der Friedrich-Wilhelm-Murnau-Stiftung wird darauf hingewiesen, dass im Ausland Die Nibelungen, vor allem über die Arbeit von Goethe-Instituten, als wichtiger Bestandteil der deutschen Kultur und des Erbes etabliert wurde. Ähnlich wie bei dem oben beschriebenen Fall von Die Schönste brachte die deutsche Wiedervereinigung auch in der Archivlandschaft Veränderungen, die Zugang zu neuem Material förderten. So beendet die für die vorliegende DVD produzierte Dokumentation ihren ,Marsch durch die Geschichte' mit 
der hier editierten Fassung auf DVD. Die editierte Restaurierung wird mit der Restaurierungsgeschichte des Films verglichen. Das heißt, frühere restaurative Eingriffe werden in die Überlieferungsgeschichte integriert und als deren Bestandteil gesehen - womit gleichzeitig auch die zeitliche Gebundenheit von Ergebnissen solcher Arbeiten deutlich gemacht wird. ${ }^{75}$

Die Nibelungen ist vor dem Hintergrund meiner Ausführungen zu sehen als ein Film der Filmgeschichte, als ein Film in der Geschichte (des Films). Das Werk steht besonders im Kontext seiner gesellschaftlichen wie politischen Gebrauchsweisen und Funktionalisierungen. Die Überlieferung ist Produkt und Faktor der geschichtlichen Entwicklung: Insofern vermittelt die Dokumentation ein Bild von Filmgeschichte, das von vornherein die Reprise, die eine politische Re-Vision zur Folge hat, mitdenkt. Als ästhetische Verfahren der Dokumentation sind vor allem die Formierungen der Bilder der Geschichte hervorzuheben, die ständig in verschiedenen Formen über die aktuelle Montage relativiert und in neuen, vervielfachten Zusammenhängen gezeigt werden. Diese Pluralität wird als Bedingung und Problem der Zugänglich- und Sichtbarkeit heute ausgestellt. Gleichzeitig, narrativiert und illustriert durch die Dokumentation, wird diese Pluralität zum Konsekrationsargument in der digitalen Edition. Durch den Paratext der Dokumentation thematisiert die DVD die Bedingungen in ästhetisch ausgeprägter Form, die hybridisierenden Verfahren verschrieben ist; hybridisierend in dem Sinne, dass die Bedeutungsproduktion über Bilder in doppelter Ebene koexistierend (nebeneinander) wirkt:

- Zum einen beschreibt das Verfahren der Hybridisierung der Register das Prinzip der Bilder- und Symbolaneignung der Nazis inhaltlich. Das ideologisch intendierte Geschichtsbild wird illustriert.

- In diesem Prozess der Illustration und damit der Reprise wiederholt die Dokumentation zum einen hybridisierende Verfahren zwischen Fakt und medialer Fiktion, die dem filmischen Material inhärent sind, und verstärkt diese noch im eigenen Montagezusammenhang (vgl. zu ähnlichen Verfahren der Legierung von Bildregistern und zu ihren Effekten $\rightarrow$ Kap. 6).

75 Für die vorliegende Fassung gilt, wie es Anke Wilkening, bis 2019 Restauratorin der Friedrich-Wilhelm-Murnau-Stiftung selbst in der Dokumentation formuliert: „[I]nsofern denke ich, ergibt sich dadurch die Möglichkeit, wirklich nochmal NibELUNGEN neu zu entdecken, weil jetzt vielleicht so richtig zum ersten Mal die Leistungen des Films in ästhetischer Hinsicht deutlich werden." Dies gilt vor allem für das besondere Tinting, die Einfärbung dieser restaurierten Version, zu der sich Wilkening auch publizistisch mit ihren Forschungsbefunden zum Umgang mit historischen Farbverfahren im Rahmen des Restaurierungsprozesses geäußert hat; vgl. Wilkening (2009). 
In dem Prozess der Reprise kommen sinnliche Schauwerte, vor allem des Lang'schen Spielfilms, zum Tragen, die die Attraktion des präsentischen Erlebnisses vermitteln. Die Form der Montage in der Dokumentation lässt die Bilder als fragmenthafte Passagen eines filmischen Spektakels wirken - im Sinne Benjamins (1982, vgl. $\rightarrow$ Kap.3). Dabei vermittelt sich eine Mise en Relation, die auf eine mit der sinnlichen Attraktion operierende Geschichtsbildmodellierung zielt und zugleich eine mehrschichtige historisierende Lektüre der Bilder initiiert. Thematisch wird die Idee eines Mythos in seinen Ambivalenzen verhandelt, der die Zeit überdauert. Allerdings wird der Mythos des Lang'schen Films von der Dokumentation nicht näher bestimmt - es bleibt letztendlich unklar, ob die Story des Films und ihr Sujet, der Film selbst als Medienereignis oder die Ikonografie der Bilder gemeint sind. So begründet die interagierende Überlagerung der Bedeutungsebenen, die Heterogenität des überlieferten Torsos, den schemenhaften Mythos, was wiederum eine Funktion in Hinblick auf die aktuelle Edition erfüllt. Die Funktionalisierung historischer und symbolischer Wandelbarkeit macht das Erzählen der Geschichte im Modus des Audiovisuellen attraktiv. Dabei wird zugleich eine interesselose Enunziationsinstanz von Geschichte etabliert. Dies ist paradox, da es ja gerade inhaltlich um die Wandelbarkeit der Sinnhaftigkeit und der möglichen ideologischen Vereinnahmung von Bewegtbildern geht. Es bleibt die ambivalente Situation bestehen, dass im kulturindustriellen Kontext der hier editierten Version die Heterogenität der Bedeutungsschichten von Bewegtbildern zum Mythos des editierten Films nachhaltig beiträgt. Gleichzeitig wird zudem über die Legierung von historisierenden Referenzen und präsentisch wirkenden filmischen Schauwerten eine interessegeleitete Narration in Hinblick auf die Restaurierungsleistung sinnlich vermittelt.

Auf einer grundsätzlicheren Ebene stellt somit die Dokumentation über ihr ästhetisches Verfahren die Relativität historischer Bedeutungsproduktion über Bilder und Konzepte geschichtlicher Zeitlichkeit auf der Erlebnisebene des Zuschauers aus - allerdings in einem Spannungsfeld, das aufzeigt, dass genau diese Komplexitäten für die heutigen Restaurierungsmaßnahmen nachhaltige Probleme darstellen. Aber - so die Argumentation der Dokumentation - diese seien mit der vorliegenden digitalen Edition (vorläufig) gelöst. So ist die Vermittlung der Clusterhaftigkeit des historischen Werks Die Nibelungen mit Blick auf seine filmhistorische Bedeutsamkeit und Kanonisierung eine zentrale Konsekrationsbedingung. Der Zuschauer wird über die heterogene Montage der unterschiedlichen Bildregister in eine Haltung versetzt, in der er ständig seine Lektüreformen - tatsächliche Geschichte referenzierend oder fiktionalisierend - vergleichend abwägen muss. Damit wird wirkungsästhetisch ein synthetisches Geschichtsbild modelliert, 
in das die mythenhaften Bilder der Spielfilme je nach Mise en Relation des Zuschauers historisierend wirken - oder aber gerade im Kontext der aktuellen Restaurierung als Ausdruck einer Fassbarmachung des Filmmythos erlebt werden - im Zeichen eines diffusen Erwartungshorizonts von Moderne.

\section{Mise en Relation der Mythen II. Metropolis (1927), ein mythisch- modernes filmhistorisches Cluster}

Der Fall Metropolis ist eines der prominentesten Beispiele der deutschen Filmrestaurierung und stellt damit ein weiteres sehr populäres Exempel eines sich in der Zeit wandelnden filmhistorischen Clusters dar: Seit Jahrzehnten ist Fritz Langs Werk Objekt restauratorischer wie rekonstruktiver Bemühungen und hat verschiedene Formen der Edition durchlaufen (Bohn 2005: 8). Dies gibt Anlass, weiterführend nach den spezifischen Ausprägungen der Mise en Relation sowie nach den umgebenden Diskurskontexten und ihren geschichtsbildmodellierenden Konsequenzen zu fragen. Dies schließt nicht zuletzt an Überlegungen im vorherigen Abschnitt an, in denen es um die clusterhafte Verwebung von Diskursen und medialen Vermittlungsformen von Zeitlichkeiten ging, die sich insbesondere bei Filmen auftun, die inhaltlich und überlieferungsgeschichtlich mit Konnotationen des mythenhaft Überzeitlichen in Verbindung gebracht werden.

In den 1980er Jahren hatte Enno Patalas sich mit seiner philologischen Quellenarbeit zu Metropolis hervorgetan und die sogenannte Münchner Fassung erstellt (Worschech 2010: 32). Seine Vorarbeiten hatten lange Zeit Referenzfunktion. 2001 folgte dann die digitale Restaurierung - mitgetragen von der Friedrich-Wilhelm-Murnau-Stiftung, der Deutschen Kinemathek in Zusammenarbeit mit der Firma Alpha Omega Digital. Mehrere Jahre war die editierte Fassung in Deutschland die bekannteste zirkulierende DVD von diesem Film. ${ }^{76}{ }_{2005}$ folgte dann die bemerkenswerte kritische Studienfassung, die auf DVD die Rekonstruktionsgeschichte strukturell in den Modus der Filmrezeption einbaute - dazu später mehr. Die Geschichte der 2010 rekonstruierten Fassung, in die das bis dahin verschollen geglaubte Material aus Argentinien integriert wurde, ist weithin in der Presse kommentiert worden. Als Basis dieser Rekonstruktion wurde das 2001 digital restaurierte Material verwendet.

Die publizistische und diskursive Rahmung dieser Rekonstruktionsgeschichte, die auch in einigen filmhistorischen Dokumentationen weiterge-

76 DVD: Friedrich-Wilhelm-Murnau-Stiftung/Transit Film, Deutsche Kinemathek 2003 $\rightarrow$ Filmverzeichnis. 
schrieben wird, zeigt vor allem eines: Das historische Werk Metropolis wird auf ganz unterschiedlichen Ebenen begriffen, narrativ konstruiert und instrumentalisiert. Die Komplexität liegt zum einen in dem industriellen, institutionellen wie ästhetischen Objekt begründet, das der (historische) Gegenstand Film darstellen kann. Über was redet man, wenn man über den historischen Film redet? Fokussiert man den Inhalt, das historische filmmaterielle Dokument oder Artefakt, die institutionellen und produktionsgeschichtlichen Umstände? Und mit wachsender zeitlicher Distanz multiplizieren sich auf allen Ebenen die entsprechenden Narrative.

Mit Blick auf die Überlieferungsgeschichte von Metropolis bedeutet dies, ${ }^{77}$ dass die immer wieder vorgenommene Zuschreibung von Modernität an das Werk zunächst auf die inhaltliche Ebene (der Story) sowie auch auf die historischen filmtechnischen Innovationen der Spezialeffekte und Tricktechniken bezogen wird. Diese beiden Ebenen werden bereits miteinander vermengt - wie anlässlich der Restaurierung 2010 noch einmal publizistisch deutlich wurde: „Alchimie für die Zukunft“ (Süddeutsche Zeitung 2010: 15). Die phantastischen Modernitätsattribute erfahren dann eine Übertragung auf die heutige Wirkung. Sie liefern in der Verlängerung der Argumentation die Begründung der heutigen Aktualität des Films und seiner Bedeutung als kulturelles Erbe. Interessanterweise scheint der Film gerade durch seine Geschichte in der Zeit seine zusätzliche Bedeutung erworben zu haben: „Bei seiner Premiere war Metropolis ein Film der Superlative. Aber noch kein Meisterwerk" (Worschech 2010: 30).

Dies wird zusätzlich überlagert von der abenteuerlich narrativierten Genese des Films sowie seiner mittlerweile nun über 8o-jährigen Fassungs- und Versionsgeschichte, deren Varianten mit dem Gestus einer archäologischen Schatzsuche nach den verschollenen Partien nachhaltig weitergeschrieben werden. Etwa werden Archivare zu Entdeckern: So erzählt Karen Naundorf 2008 eindrücklich in ihrer Reportage zu der „Reise nach Metropolis“:

Paula Félix Didier hatte geahnt, dass ihr niemand glauben würde. Sie saß an dem Schreibtisch in ihrem kalten Büro in Buenos Aires und wartete auf eine Mail aus Deutschland. Doch es kam - nichts. Warum auch sollte irgendein Experte glauben, dass sie [...] gefunden hatte, wonach Forscher und Restauratoren seit Jahrzehnten vergeblich in den Archiven der Welt suchten? [...] Aber in der kleinen Kammer hinter der grünen Metalltür gleich neben ihrem Büro lagen sie: drei große Rollen, vorsichtig in silbrig schimmernden Blechdosen verstaut (Naundorf 2008). 
Eine digitale, ideelle, zeitlich vorgelagerte Reaktion auf diese grundsätzliche Problematik ist die bereits erwähnte kritische Studienausgabe von Metropolis aus dem Jahr 2005. Sie übersetzt die Werkgenese und Überlieferungsgeschichte in eine „digitale Wissensarchitektur“ (Völckers 2005: 4). Strukturell heißt dies, dass in dieser Ausgabe nicht mehr eine lineare Auffassung von Filmgeschichte und dem Werk vorherrscht, sondern ein zeitliches Konzept von Simultaneität umgesetzt wird: Über horizontale und vertikale Rezeptionsmöglichkeiten können die clusterartigen Überformungen der Zeit und auch in der Zeit als Verweisungsstruktur über die DVD-Architektur erfahren werden. In der literarischen Editionsgeschichte sind solche Verfahren schon lange etabliert. Für das Medium Film existiert aber - so betont Friedemann Beyer von der Friedrich-Wilhelm-Murnau-Stiftung 2005 - bisher keine standardisierte vergleichbare Methode (Beyer 2005: 5).$^{78}$ Die kritische Studienausgabe versucht nun im Jahr 2005, gerade über das Medium der DVD ${ }^{79}$ die Arbeit der Rekonstruktion und Filmhistoriografie zu problematisieren:

\begin{abstract}
Die Arbeit der Rekonstruktion ist [...] wesentlich auch die des Erkennens eines komplexen, prozesshaften Geschehens, das es auf einer Zeitschiene - linear und zugleich simultan - zu begreifen gilt. Die Konsumentenvorstellung einer in geregelten Bahnen und in abgeschlossenen Einheiten rekonstruierbaren Filmgeschichte muss zwangsläufig enttäuscht werden; es kann sie logisch gar nicht geben (Emigholz 2005: 3).
\end{abstract}

Insbesondere mit den digitalen Möglichkeiten beschleunigt sich die Tendenz: „Alte Filme, neue Lektüre“ - so lautet Enno Patalas' (2005) Überschrift zu dem Problem, dass digitale Technologien die Komplexität grundsätzlicher Fragestellungen, die sich per se schon beim Film ergeben, steigern. Autor, Werk, Original - diese Grundbegriffe des bürgerlichen Kunstdenkens seien - so Patalas - durch die auf technischer Reproduzierbarkeit basierenden Medien mächtig ins Rutschen gekommen. Vor allem beim Film habe man es weniger mit Werken als mit Serien zu tun: mit Fassungen, Kopien, Aufführungen

${ }_{78}$ Beim Film gab es zu dem Zeitpunkt (2005) oft nur quellen- und editionskritische Informationen und Dokumentationen in Form schriftlicher und grafischer Rekonstruktions- wie Restaurationsberichte - und hier muss man bei der Quellenlage bedenken, dass es sich um Arbeitsberichte von Firmen handelt, die mit ihrem Produkt und ihrer Handfertigkeit werben wollen. Das heißt, die hergestellte Version muss eine gewisse materialinhärente Zwangsläufigkeit vermitteln.

79 Vgl. grundsätzlich zu anderen, jüngeren Formen der historisch-kritischen Filmedition in digitalen Medien am konkreten Beispiel des Werks von Dziga Vertov - auch mit wissenschaftsgeschichtlicher Reflexion von jüngeren Digital-Humanities-Ansätzen: Heftberger (2016). 
(Patalas 2005: 6). Wie bereits einleitend zu diesem $\rightarrow$ Kap. 7 ausführlicher kontextualisiert und theoretisiert, stellen diese Begriffe und Problemstellungen tatsächlich die kritischen Dreh- und Angelpunkte für die Erfahrung des Status eines Kunstwerks in der digitalen Domäne dar.

Die kritische Studienfassung von Metropolis sticht hier hervor. Denn man hat sich für die Edition eines Torsos entschieden. ${ }^{80}$ Sie legt offen, dass das filmische Werk nicht vollständig überliefert ist. MEт ROPOLIS wird bewusst eingedenk seiner tatsächlichen Überlieferungsgeschichte - als Fragment belassen. Mit meiner Terminologie könnte man dies auch als einen Versuch beschreiben, für den Film eine alternative „Ästhetik des Fragments“ (Bohn 2013b: 297-314) zu entwerfen - mit ambivalenten Folgen für die konkrete Filmerfahrung: Die Fehlstellen werden als solche deutlich gekennzeichnet und mit Verweisen auf mögliche außerfilmische Quellen optional angereichert. Das bewusst von dem anschaulichen Begleitheft reflektierte Konzept der Lacunae wird aus den Editionswissenschaften übernommen (Bohn 2005: 9). ${ }^{81}$ Damit wird sich einer linearen Konstruktion des ästhetischen Textes, die die Fragmenthaftigkeit der Quellenlage und des Wissenstandes um die fehlenden Bilder verschleiern würde, verweigert. Man akzeptiert, dass das ästhetische Er-

8o Ein weiteres interessantes Beispiel für die digitale Edition von Fragmenten etwa aus derselben Zeit (2006) wie die Metropolis-Studienfassung stellt die DVD-Edition von Charles Taits The Story of the Kelly GaNg aus dem Jahr 1906 dar (National Film and Sound Archive Australia). Der Film gilt als einer der weltweit ersten narrativen Langfilme. Er wurde lange als verschollen behandelt, ab den 1970er Jahren tauchten aber immer mehr Fragmente des Films auf - zusätzlich zu weiteren nichtfilmischen Quellenmaterialien wie Programmheften und Filmpostern. All diese Quellen wurden in der Edition - zusammen mit einer digitalen Restaurierung des Fragments - zusammengeführt. Von dem ursprünglich rund einstündigen Film sind etwa 17 Minuten Bewegtbilder erhalten. Auf der DVDEdition von 2006 sind die Fragmente als Restored Version zu sichten. Zur Auswahl stehen hier zwei verschiedene Musikbegleitungen, eine davon modern-experimentell. Darüber hinaus wird vom Menü auch eine STUDY VERSION angeboten, die dem Projekt der Studienfassung von Metropolis in gewisser Weise ähnelt: Hier wird versucht, die Narration beziehungsweise den narrativen Rhythmus mit der Markierung der entsprechenden Lücken zu rekonstruieren und damit auch einen Eindruck der eigentlichen Dauer des Films erfahrbar zu machen. Die Lacunae sind zum Teil mit ergänzten - vom Archiv als solche ausgewiesenen - rekonstruierten Zwischentiteln und auch anderen Quellen gekennzeichnet. Zusätzlich kann man diese Version mit einem kuratorischen Kommentar anschauen.

81 Vgl. hier auch den restaurationsethischen Umgang mit antiken Statuen: Bis zum 19. Jahrhundert war es üblich, die verstümmelten Statuen mit Ersatz-/Füllmaterial zu komplettieren. Heute ist die gängige Praxis in kunstwissenschaftlicher Restaurierung, dass nicht eine ,Ergänzung' oder ,Komplettierung‘ erfolgt, sondern die Praxis besteht in der Konservierung des Torsos als Fragment ohne imaginative Ergänzungen (vgl. auch ausführlich Bohn 2013a und 2013b). 
lebnis in der Zeit eine (nonlineare) fragmentierte Geschichte hat. So wird filmhistorisches Arbeiten offen gelegt und digital editiert. Allerdings tritt damit die ästhetische Filmerfahrung von Langs Film nachdrücklich in den Hintergrund. Der Modus des Vergleichens verlegt sich für den Nutzer/Rezipienten vollkommen in den Dienst eines kognitiven, quellenphilologisch orientierten Erkenntnisgewinns. Der zeitliche Fluss des Mediums Films in seiner linearen Form der Entfaltung wird unterminiert.

Historiografisch ist interessant - und hier hat die Causa MEtropolis sich selbst überholt -, dass nun die Fassung von 2005 ein Zeitdokument über die Forschung und das Wissen in jenem Jahr ist. Damit und mit dem technologischen Stand sowie den restaurativen und rekonstruktionseditorischen Entscheidungen schreibt sich der Moment der DVD-Produktion in die Filmhistoriografie ein. Im Sinne Deleuzes könnte man wissenschaftshistorisch fast feststellen: Hier liegt ein digitales Zeugnis vom filmhistoriografischen Wissensstand von 2005 vor - in Form eines clusterförmigen, auf Simultaneität bauenden Zeitbildes. Das hier vermittelte Wissen setzt sich aus verschiedenen (Forschungsstand-)Schichten und Bildbearbeitungsphasen zusammen.

Die Studienfassung stellt somit ein Beispiel dar, in dem das Cluster Metropolis mitsamt seiner Überlieferungsgeschichte und seinen diskursiven Zuschreibungen eine Konsekration erhält, die sich im Feld einer akademischen Editionsphilologie bewegt. Vor diesem Hintergrund tritt aber die sinnliche Erfahrung von Fritz Langs Film zurück - zugunsten einer medialen Konfiguration der Überlieferungsgeschichte im Zeichen einer Mise en Relation des Nebeneinanders heterogener Quellen. Damit ist die DVD aus dem Jahre 2005 ein Zeugnis der Konsekration des Films als Kulturerbe im akademischen und kulturpolitischen Diskurs. Darüber hinaus wird der Mythos der Überlieferungsgeschichte des Clusters Metropolis um eine Ebene erweitert - namentlich um das Diskursfeld neuerer, moderner, da zukunftsversprechender digitaler Restaurierungs- und vor allem Editionsmöglichkeiten. Insbesondere Letztere werden wohl in nächster Zeit noch vermehrt im Kontext der sich entwickelnden sogenannten Digital Humanities in konkreten Praktiken weitere Ausformulierungen finden. ${ }^{82}$

Gerade auch unter der Maßgabe meines Ansatzes wäre bei dieser Zukunftsprognose mein Plädoyer - auch in Anbetracht des Falles Metropolis -, bei Filmanalysen die Frage nach der Re-Konfiguration filmästhetischer

82 An dieser Stelle sei weiterführend erneut auf Heftberger (2016) hingewiesen, die sich in einer Monografie detailliert mit den Möglichkeiten einer von Digital Humanities geleiteten Analyse und der Publikation von filmhistorischen Erkenntnissen am Beispiel des Werks von Dziga Vertov auseinandersetzt. 
Erfahrungen in neuen (Untersuchungs-)Kontexten nicht aus den Augen zu verlieren und spezifische Fragen der Bewegtbildwahrnehmung in neu kreierten (Wissens-)Dispositiven weiterhin im Blick zu behalten; dies zumal auch unter dem Vorzeichen, dass diese Dispositive in der digitalen Domäne Elemente einer zeitlich sehr dynamischen (technischen) Entwicklung sind. Vor diesem Hintergrund ist eine kritische Perspektive auf die jeweiligen Ausprägungen und Konfigurationen von Wahrnehmungsdispositionen einer Mise en Relation - auch jenseits von den hier besprochenen Dispositiven wie DVD respektive Blu-Ray-Disc - von großer Bedeutung. ${ }^{83}$

\subsection{Körperlich-gestisch erlebte Mise en Relation. Split Screen und Do-it-yourself-(DIY-)Restaurierungen}

Split-Screen-Konfigurationen habe ich bereits mehrfach in unterschiedlichen Kontexten als ästhetische Vermittlungsform von medien- und filmhistoriografisch wirksamer Erfahrungsbildung beschrieben. Solche Bildkonfigurationen funktionalisieren den Zuschauer als performative (Nach-)Vollzugsinstanz. Die Ein- und Anbindung des Zuschauers geschieht entprechend meinen Feststellungen in $\rightarrow$ Kap. 5 sowohl kognitiv wie vor allem phänomenal. Meine Modellierung rekurriert hier auf Konzepte der Filmphänomenologie, die das Verhältnis zwischen Zuschauern und Bewegtbildern entwerfen.

Im Folgenden soll es um eine dispositivische Variante von Bildkonfigurationen gehen, die dem Split-Screen-Verfahren strukturell ähnlich ist. In diesem Zusammenhang behandelt der vorliegende Abschnitt den historiografisch wirksamen Prozess einer Mise en Relation; ein Prozess, in dem der Modus des Vergleichs sich dezidiert in einer tatsächlich körperlich-gestisch (nach)vollzogenen zeitlichen Differenz vermittelt, die ich an dieser Stelle als Ausprägung einer erlebten Mise en Relation konzeptualisiere.

83 Zur Erinnerung an eine wichtige Voraussetzung meiner Studie (vgl. $\rightarrow$ Kap. 1): Auch wenn mein Fokus gegenständlich auf der Untersuchung der zentralen Phase der Transition analog/digital in den letzten 25 Jahren liegt, so vertrete ich die These, dass hier praktische Grundlagen und -strukturen für die Imagologie, diskursiv-ideologische Überformung, Nutzung, Anwendung und Rezeption von Filmen in der digitalen Domäne gelegt wurden. Die in dieser Phase etablierten Praktiken und Phänomene sind nicht zu vernachlässigen für die Entwicklungsgeschichte einer digitalen Kultur, in der Bewegtbilder einen wichtigen Bestandteil ausmachen. Insofern lassen sich grundlegende methodologische Systematiken, wie sie meine Studie entfaltet, auch auf zukünftige Entwicklungen digitaler Dispositive von Filmen anwenden. 
Entsprechende Phänomene lassen sich bei jüngeren medialen Vermittlungsformen von Film- wie auch von Fotogeschichte beobachten. So erschien etwa im Juli 2016 auf Spiegel Online ein fotogeschichtlicher Artikel mit dem Titel „Plötzlich ist die Welt ein bisschen bunter" (Grothe 2016). Er war ein Beitrag zur Arbeit eines amerikanischen Archivars, der historische Schwarz-WeißFotos mittels eines Computerprogramms nachkolorierte.

Zur Erinnerung (vgl. hierzu auch die Ausführungen in $\rightarrow$ Kap. 2): Die nachträgliche Kolorierung von Archivmaterial ist kein neues Phänomen. Insbesondere seit der Colorization-Debatte bei Spielfilmen in den 1980er Jahren entfachten sich um die elektronisch vorgenommenen nachträglichen ästhetischen Eingriffe in historisches Bildmaterial ausgeprägte und bis heute emotional geführte memopolitische, ethische und denkmalpflegetheoretische Debatten. Neben den in $\rightarrow$ Kap. 2 besprochenen Beispielen aus der amerikanischen Fernsehindustrie wurde in Frankreich diese Diskussion anhand der Serie APOCALYPSE über den Zweiten Weltkrieg geführt (Clarke/ Costelle, Mini, TV-Serie 20og; vgl. u. a. Psenny 2014). In Deutschland gab es 2012 einen ähnlichen Fall mit einem breiten Medienecho im Kontext von Guido Knopps Weltenbrand (vgl. die Ausführungen hierzu in $\rightarrow$ Kap. 2). Jüngst brandete die Debatte international angesichts von Peter Jacksons Umgang mit Archivmaterial aus dem ersten Weltkrieg in ThEY S HALL NOT GROW OLD (2018) wieder auf (F. Heller/Ruedel 2019).

An dieser Stelle soll allerdings ein besonderes Augenmerk auf die mediale und dispositivische Vermittlungsform der Problematik von Nachkolorierung gelenkt werden: Denn der eingangs zitierte Beitrag in der Internetzeitschrift Spiegel Online (Grothe 2016) ist mit den im Text erwähnten Fotos illustriert, die interaktiv vom Nutzer veränderbar sind. Sie enthalten die Bildunterschrift und damit die Aufforderung an den Leser/Nutzer: „Vorher - nachher: Verschieben Sie den Regler auf dem Bild." So lässt sich etwa das Bild mit dem Titel Mai 1945. Amerikanische Truppen befreien das KZ Wöbbelin bei Ludwigslust von Schwarz-Weiß zu Farbe mittels eines vom Cursor geführten Striches verändern - mit spielerischem Wischen kann man die Bewegung von links nach rechts (von Schwarz-Weiß zu Farbe) und zurück von rechts nach links (von Farbe zu Schwarz-Weiß) vollziehen.

So findet sich hier medial in einer Handbewegung umgesetzt, worum es inhaltlich in dem Artikel geht: den Prozess der Veränderung - und die kontroverse Debatte darum, ${ }^{84}$ in deren Mittelpunkt die diskursive Prämisse steht, „[d]as Bild als Farbphoto glaubhaft [zu] machen“ (Grothe 2016). Die

84 In diesem Zusammenhang zitiert der Artikel Anton Holzer (Fotohistoriker, Herausgeber der Zeitschrift Fotogeschichte): „Die nachträgliche [digitale, FH] Kolorierung historischer 
editorische Aufbereitung dieser Diskussion von Spiegel Online über die Webseite verlagert die Frage ,Wird das Bild lebendiger? Historisch glaubhafter?' in die Entscheidungshoheit des lesenden Nutzers, der im Prozess des Bildveränderns graduell seine Wahrnehmungsveränderung vergleichen kann.

Hinter der Frage nach ,Glaubhaftigkeit' verbirgt sich nichts anderes als das Mehrwertversprechen, dass das Bild einer historischen Referenz zugeordnet wird. Dies erfolgt als ästhetische Strategie über die Konditionierung heutiger Wahrnehmungsmuster, die Bewegtbildern in Farbe eine höhere mimetische Qualität zuschreiben. Dies liegt auch in der Konditionierung von sinnlicher Unmittelbarkeit der Bildwirkung, die hier mit einer interaktiven Handbewegung verbunden wird: Zentral an der Präsentationsform der Bilder ist die Vermittlung des Veränderungsprozesses unter Einbindung des nutzenden Rezipienten. Medial wird dies erfahrbar gemacht, indem der Spiegel-Online-Leser als Nutzer die Veränderung tatsächlich gestisch (nach-)vollzieht. Es geschieht eine Geschichtsbildmodellierung im Prozess der Variation des Bildes. Was inhaltlich über die nachträgliche Kolorierung durchaus kritisch diskutiert wird Wünsche und Sichtweisen vergangener Zeiten lebendig machen zu wollen -, wird über die Vermittlungsform in den präsentischen Handlungsprozess des Nutzers verlegt, er wird aktiv Teil der medienhistoriografischen Erfahrungsbildung. Das Prinzip des Performativen ( $\rightarrow$ Kap. 5 ) wird mit diesem Beispiel über mein bisheriges Modell einer filmphänomenologisch verstandenen Interaktion mit Bewegtbildern hinausgehend - mit Blick auf die tatsächlich körperlich-leibliche Handlungsgeste erweitert. In den bisherigen Beispielen zu Split-Screen-Verfahren in den anderen Kapiteln (etwa $\rightarrow$ Kap. 4 und auch in dem vorliegenden $\rightarrow$ Kap. 7, vgl. insbesondere Analysen zu Dressed то Kill oder Die Nibelungen) wurden stets nur Vergleichsformationen innerhalb von Filmen untersucht. Die an dieser Stelle behandelten Beispiele gehen in den Möglichkeiten der Interaktivität darüber hinaus. Die hier formulierten Überlegungen führen damit die bereits in $\rightarrow$ Kap. 6 diskutierten Problemstellungen der Modellierung des Verhältnisses zwischen (sinnlich) wahrnehmendem, erfahrendem Subjekt und Bewegtbildern weiter, in denen es um das zentrale Spannungsverhältnis zwischen den folgenden Polen geht: Auf der einen Seite wird Geschichte verstanden als Wahrnehmungseffekt, wobei sich dies unter anderem nach Steinles Konzept (2005) nachhaltig mit der Annahme einer abstrakten, unpersönlichen (Enunziations-)Instanz verbindet.

Auf der anderen Seite formiert sich aber das phänomenologisch gedachte Subjekt, das relational über sein zeitliches Erleben im Wahrnehmungsakt Teil

Fotos hingegen verändere die Bildaussage - wir überziehen sie mit einer Lasur heutiger Wahrnehmungswünsche." 
dieser Instanz wird. Die hier angeführten Beispiele spitzen das Vexierspiel zwischen Geschichte als medialem Wahrnehmungseffekt und Geschichte als medial-leiblichem Vollzugseffekt (wenn nicht gar-affekt) zu - mit besonderem Fokus auf Modi einer Mise en Relation.

Wie sehr in einer solchen Anordnung die Grenzen zwischen der Geschichte von Filmbildern und Geschichte an sich, vermittelt als diffuses Erleben zeitlicher Differenzen anhand von Bildern, memopolitisch wirksam verschmelzen, zeigen Konfigurationen auf der Webseite der amerikanischen Film Foundation im Jahr 2016, die denen auf Spiegel Online ähneln. ${ }^{85}$ Im Zuge ihres dort formulierten Leitfadens zur allgemeinen Bedeutung von Filmsicherung präsentiert die Stiftung zur Illustration eine sogenannte Do-it-yourself-(DIY-) Filmrestaurierung. In diesem Kontext zeigte die Webseite lange Zeit Filmstills aus prestigeträchtigen Restaurierungen (zu Beginn 2016 ist es etwa ein Bild aus The Life and Death of Colonel Blimp von Powell/Pressburger, 1943; Abb. 7.34). Auch hier konnte man mit dem Cursor über das Bild wischen und es damit vor allem farblich ,restaurieren'.

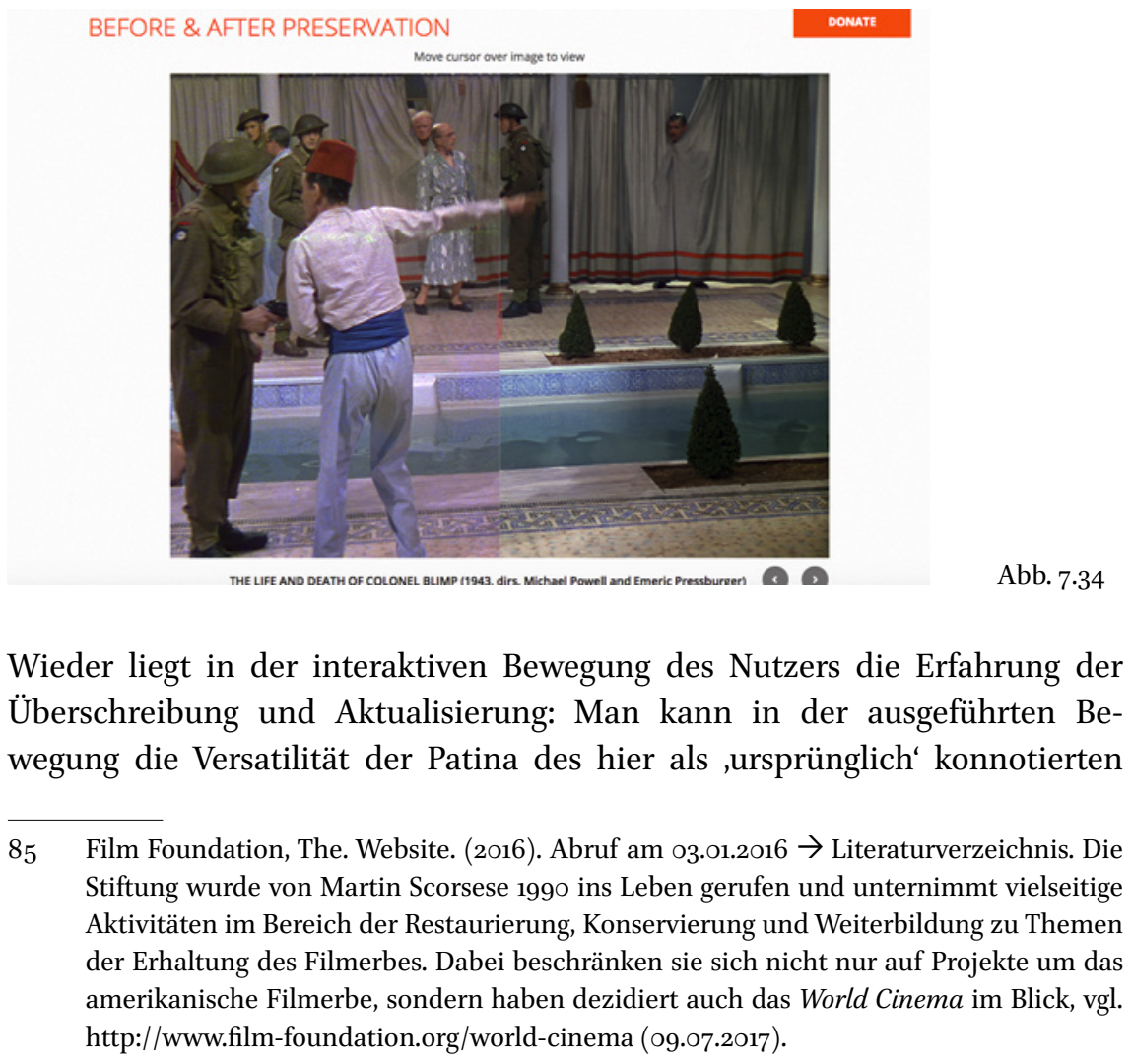


Bild-/Filmzustands wahrnehmen, man erlebt vergleichend den Prozess der Veränderungen - deren Zeitlichkeit man selbst bestimmt. In einem Bild wird die notwendige Gleichzeitigkeit und Relationierung von Altem und Neuem, zwischen Aktuellem und Restaurierungspotenzialen zum Ausdruck gebracht.

Die mediale Anordnung lässt es allerdings zu, dass man als Nutzer spielerisch die Veränderung rückgängig machen kann, dass man die Bewegung wiederholend hin und zurück ausprobieren und damit den Veränderungsprozess in seiner Wirkung immer wieder erproben kann. Vor- und Rückwärtsbewegung in der Zeit koexistieren potenziell in einem Bild, jeder Zustand ist nur ein vorläufiger. Somit wird im Bearbeitungsprozess der Bilder dessen Relativität körperlich-gestisch erfahrbar. Deshalb spreche ich oben auch in Hinblick auf das Wischen mit dem Cursor von einem gestisch-manifesten (Nach-) Vollzugsaffekt.

Die offizielle Webseite des Archivfilmfestivals Il Cinema Ritrovato erklärte 2016 und 2017 mit ähnlichen Wischbildern das Prinzip von Filmrestaurierung (Abb. 7.35).

Abb. $7 \cdot 35$

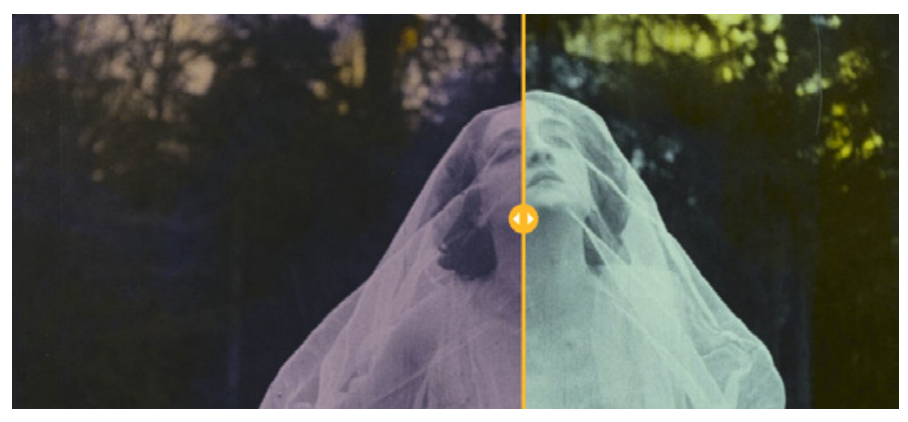

Mit der Beschreibung, man gebe den Filmen in der Restaurierung „[a] new life“, ${ }^{86}$ legierte die Webseite meines Erachtens die Vorstellung eines in der Restaurierung wiederbelebten Filmwerks mit der interaktiven Veränderungsmöglichkeit des Bildes seitens des Internetusers (Abb. 7·36-7·37).

In diesem Horizont erhielt auch der begleitende Paratext der Webseite eine Bedeutung auf der inhaltlichen wie rezeptionsästhetischen Ebene, wobei grundsätzlich die zeitliche Relativität von Restaurierungen respektive

86 Vgl. Cinema Ritrovato, Il. Website. Restoration. (2016). $\rightarrow$ Literaturverzeichnis. Weitere interessante, ähnliche Beispiele liefert die Webseite des niederländischen Eye Film Museums in Amsterdam zur Vermittlung seiner Arbeit; etwa das für junge Leute entwickelte Film restoration activity programme, vgl. Eyefilm.nl. Website. Restoration and Digitization (o. J.) $\rightarrow$ Literaturverzeichnis. 

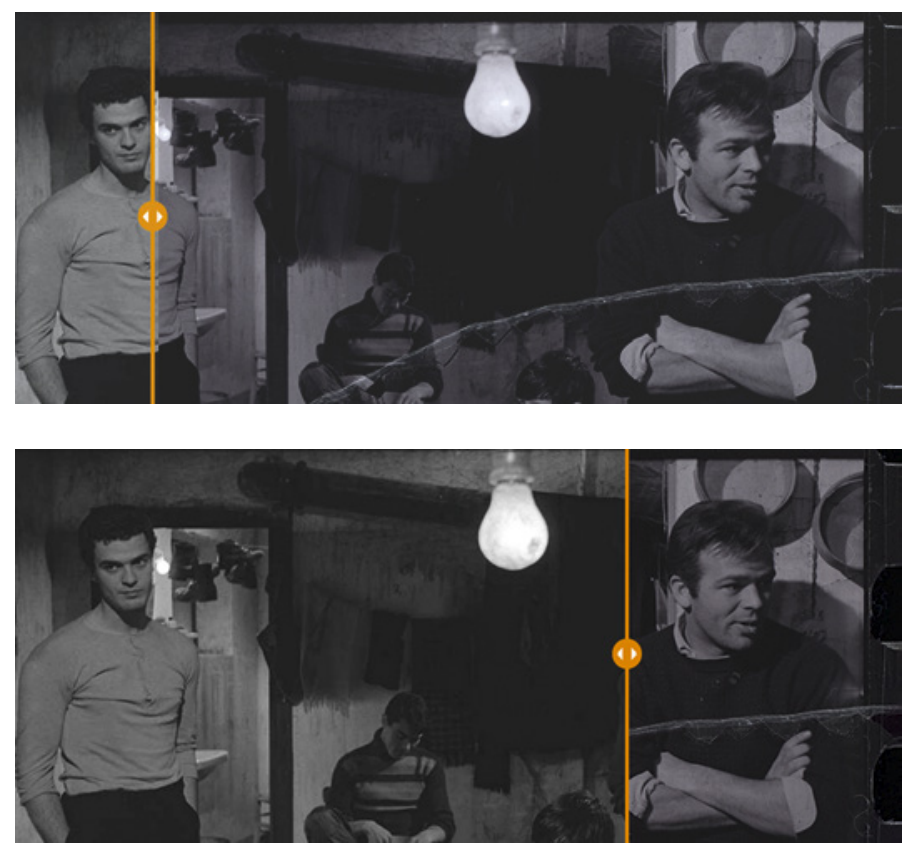

Abb. $7 \cdot 36$

Abb. $7 \cdot 37$

digitalen Bildbearbeitungen sowie die Abhängigkeit von den jeweils beteiligten Subjekten hervorgehoben wurden: „Every restoration is a child of its time. It is subject to the limitations and possibilities of the technologies employed, but also to the interpretation of the work by those individuals carrying out the restoration" (Cinema Ritrovato, Il. Website. Restoration. 2016). ${ }^{87} \mathrm{Im}$ übertragenen Sinne auf die Rezeptionsform der medialen Vermittlung von Filmrestaurierung bezogen, könnte man formulieren, dass die Einbeziehung des Internetnutzers in die Bewegung der Veränderung ideell zumindest ansatzweise auf die besondere Bedeutung des Subjekts für die Bestimmung der historischen Position und Erscheinungsweise der Bilder hinweist.

Somit lassen solche Wischbilder als mediale Vermittlungsform von Filmgeschichte emblematisch in ihrer Anordnung das Grundproblem manifest werden: Ausgehend von der schon in $\rightarrow$ Kap. 2 entwickelten Position, dass jede Erscheinungsform von digitalen Bildern - vor allem auch die ehemals analoger Bilder, welche nun digitalisiert und restauriert werden - das Resultat eines situativ, in der Gegenwart verhafteten Interpretationsprozesses darstellen, muss für die vermittelten Geschichtsbildmodellierungen eine stets

$87 \quad$ Abruf am 21.07.2016 $\rightarrow$ Literaturverzeichnis. 
prekäre Vorläufigkeit integral mitgedacht werden. Es sind nachhaltig über den augenblicklichen (technologischen, ökonomischen) Kontext und über situative Entscheidungsprozesse definierte Manifestationen von Bildern. Dies gilt im Besonderen für die Ergebnisse von Filmrestaurierungen. Die Möglichkeit einer zukünftigen Veränderung und Wiederbearbeitung ist immer schon (spielerisch-potenziell) vorhanden. ${ }^{88}$ Die Wischbilder, die über eine Handbewegung zeitliche Veränderungen historischer Zustände von Bildern thematisieren, stellen ein interessantes Medium dar, welches über seine Nutzungsform zeit- und geschichtstheoretische Dimensionen implizit erfahrbar macht. Dabei werden allerdings auch die Widersprüche von Bildkonfigurationen deutlich, die mit der diskursiven und wirkungsästhetischen Legierung der Ebenen a. Geschichte mit Bildern, b. Geschichte der Bilder und c. Bilder in der Geschichte entstehen. Die Legierung dieser Ebenen hat eine Diffundierung des eindeutigen historischen Bezugs zur Folge.

So gilt bei diesen Wischbildern: Das vollziehende Subjekt wird in den mehrschichtigen historiografischen Prozess mit der körperlichen Geste der Handbewegung und der entstehenden Bildvariationen zeitlich eingebunden. Gleichzeitig wird die Enunziationsinstanz Geschichte dem Bild von seiten des Nutzers zugeschrieben. Es entsteht damit das Paradox einer Do-it-yourself-(DIY-)Geschichte. Das Paradox entfaltet sich im Spektrum der Wahrnehmungspole zwischen Fremdheit und Nähe im Ausprobieren der graduellen Veränderung etwa vom schwarz-weißen Archivbild zum farbigen Bild beziehungsweise vom zerstörten zum restaurierten Bild. Man kann hier von einer dispositivisch-medialen Variante des historiografisch wirksamen Erfahrungsprinzips Bridging the gap, marking the difference sprechen ( $\rightarrow$ Kap. 5 ). Oder anders ausgedrückt: Dieses operative Verfahren der Bildbearbeitung und deren Veranschaulichung erscheinen im gestisch-körperlichen Nachvollzug als verzeitlichte Varianten dessen, was im Split-Screen-Verfahren statisch konfiguriert wird.

88 Die zeitliche Dimension, die in solchen Bildkonfigurationen konzeptionell, auf geschichtstheoretischer Ebene zum Ausdruck kommt, könnte man mit dem zeitphilosophischen Begriff des Werdens von Gilles Deleuze beschreiben (Deleuze 1997a und b). Das Konzept des Werdens bedeutet eine Prozessualisierung der Denkformen. Angewendet auf die Implikationen der interaktiv veränderbaren Bilder und in Hinblick auf die verschiedenen temporalen Referenzen und Zustände lassen sich die Schichtungen von koexistierenden Zeiten, in denen Vergangenes und Kommendes immer im Gegenwärtigen schon kopräsent sind, analytisch fassbar machen. 
Es ist bereits mehrfach auf das ästhetische Potenzial von Split-ScreenKonfigurationen hingewiesen worden, insbesondere mit Blick auf deren Wirkungsdimension, in welcher der Zuschauer zur Nachvollzugsinstanz wird, die gleichsam als sinnliche Entscheidungsinstanz im kulturindustriellen Interesse funktionalisiert werden kann. Solche Konfigurationen stellen bedeutsame Exempel von Verfahren der Mise en Relation im Dienste medienhistoriografischer Erfahrungsbildung dar. Vor diesem Hintergrund soll deshalb in einem kurzen Exkurs zum Split Screen das Phänomen theoretisch verortet werden - damit werden bereits an den Beispielen ausgemachte Befunde ergänzend vertieft (Abb. 7.38) ${ }^{89}$.

magazin play $^{4}$

Die große Remaster-flut

Mit Odin Sphere und Kick Off haben es zwei Remakes bereits in diese Ausgabe geschafft - die richtig dicken Revival-Brocken kommen aber erst noch...

The Elder Scrolls 5: Skyrim - Special Edition

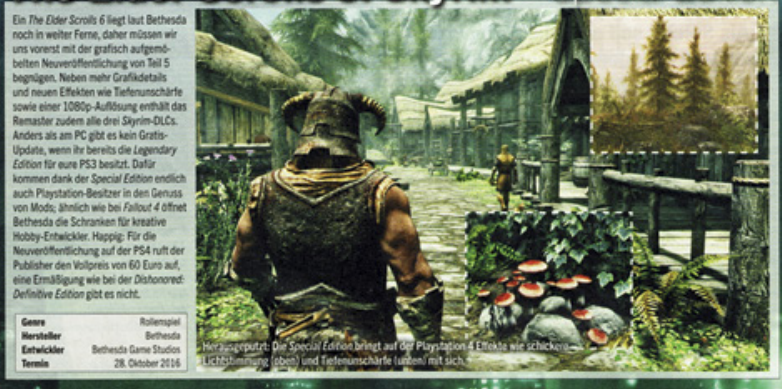

Bioshock; The-Collection :-

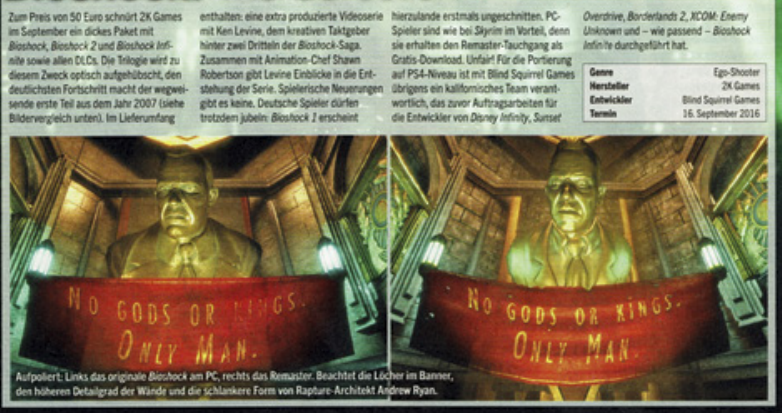

$90 \quad 09.2016$ in ing

momolawiard:

Abb. $7 \cdot 38$

89 Grundsätzlich kann man diese Konfiguration auch für andere Medienprodukte feststellen. Etwa bei Computerspielen stellt sich die Frage nach dem ,Remastering' noch 
Überdies wird unten die wirkungsästhetische Dimension methodologisch und wissenschaftsgeschichtlich eingeordnet. Letzteres ist vor allem für Fragen dispositivischer Anordnungen von Relevanz.

Insbesondere der Filmwissenschaftler Malte Hagener (u. a. 2008) hat zur mediengeschichtlichen Bedeutung und Einordnung des Split Screens gearbeitet. Unter anderem in seinem Text The Aesthetics of Displays. How the Split Screen Remediates Other Media (2008) bezieht sich Hagener auf die remediierenden Qualitäten von Kino, indem er eine Genealogie des Gebrauchs von Split Screens in Reaktion auf andere Medienentwicklungen wie Telefon, TV oder CCTV als digitale Bilderscheinungsweisen beschreibt. Wenn Hagener im Gebrauch des Split Screens immer einen inhärenten Metadiskurs zwischen Immersion in die Illusionsbildung der Bilder und dem Hinweis auf die artifizielle Natur des Filmbildes ausmacht, ${ }^{90}$ so gibt es Anschlussmöglichkeiten an den vorliegenden Gegenstandsbereich. Im Restaurierungsdiskurs bewegen wir uns immer schon auf einer Metaebene, die Film, vornehmlich im Zeichen digitaler Medienentwicklungen, retrospektiv vor allem über die Materialebene, dann aber auch in seinem Kunstwerkcharakter und in seinen ästhetischen Eigenschaften zu bestimmen sucht.

Hageners Argumentation ist deshalb interessant, weil er versucht, die Brüche, die der Split Screen mit Regeln der Fiktionsbildung verursacht, einzuordnen und medienhistorisch zu erklären. Er denkt über den Zusammenhang von Form, Stil und zugleich fiktionalem Inhalt und wirkungsästhetischer Dimension nach. Daran lehnt meine Vorgehensweise an, um Split-ScreenKonfigurationen - die entstehenden Vergleichsbilder - nicht nur als Illustration eines (materialästhetischen) Differenzverhältnisses zu sehen, sondern auch Angebote der Illusionsbildung und Immersion in eine fiktionale Welt über den Bildinhalt als filmische Erfahrung für den Rezipienten mit zu berücksichtigen.

Grundsätzlich hat die Konfiguration eine Wahrnehmungsmodellierung zur Folge:

A frame within a frame draws attention to the act of framing itself by visibly displaying the basic principle that forms the condition of possibility for the

deutlicher, da ,ältere' Spiele in immer neueren Umgebungen und Konsolen gespielt werden wollen, damit sie den sich ständig wandelnden ästhetischen Ansprüchen der Spieler an den immersiven Charakter genügen. Vgl. exemplarisch die Darstellung von der „Flut von Remastered-Versionen“ im play4-Magazin (o9.2016: 9o) für die Playstation von Sony (Abb. 7.38). Auch in diesen Kontexten wird immer wieder in der Promotion mit Split-Screen-Konfigurationen gearbeitet.

9o Eigentlich stellt der Split Screen eine ,Gefahr' für die immersiv wirkende Continuity im diegetischen Universum dar: Er unterminiert „the seemingly unmediated display of story information because it foregrounds the artificial nature of the image“ (Hagener 2008: $1 \mathrm{FH})$. 
image: the frame draws a distinction between inside and outside, between image and non-image (Hagener 2008: 1-2 FH) ${ }^{91}$

Dies habe ich etwa in $\rightarrow$ Kap. 4 am Beispiel des Werbeclips zur DVD-Edition von Peter Pan erläutert: Vorher und Nachher sind in einem umgebenden Filmframe vereinigt. Unterschiedliche zeitliche Zustände werden gleichzeitig dargestellt und ohne eine weitere Referenz als Differenzverhältnis aufeinander bezogen.

Wenn in einer filmhistorischen Dokumentation oder in digitalen Wissensarchitekturen Bilder in einer Form vergleichend aufeinander bezogen werden, wie es etwa in DAS ERbe Der Nibelungen (2011), bei der Blu-Ray-Funktion der Edition (2007) von Close Encounters of the Third Kind oder bei der Studienfassung (2005) von Metropolis der Fall ist, dann ergibt sich die Relation Bild-Nichtbild, wie sie Hagener (2008) nennt. Bei einem solchen medial inszenierten Versionenvergleich werden nun zwei eigentlich räumlich getrennte filmische Objekte (zum Beispiel zwei Filmversionen beziehungsweise stellvertretend zwei Filmkader aus den jeweiligen Versionen) ästhetisch aufeinander bezogen. ${ }^{92}$ Die Dimensionen des Memopolitischen und Kulturindustriellen liegen dann in der situativen bildlichen Anordnung, die bestimmt, wie die Bilder zueinander in Bezug gesetzt werden. Dies ist, wie oben mehrfach betont, aber nicht nur auf die Materialästhetik zu beziehen, sondern der Bildinhalt grundiert zusätzlich den bildlichen Eindruck. Das an seinen konkreten Analysebeispielen festgestellte Wechselspiel zwischen Präsentem und Absentem, Aktuellem und Virtuellem beschreibt Hagener (2008: $2 \mathrm{FH}$ ) angesichts von Split-Screen-Konfigurationen als spielerisches Verhältnis in den Raum- und Zeitkonstellationen: Es gebe eine An- und Abwesenheit sowohl in der Diegese wie aber auch in der Situation des Zuschauers zwischen Nähe und Distanz zu den filmischen Ereignissen. ${ }^{93}$ Angewendet auf Restaurierungsdokumentationen kann dies ein spielerisches Verhältnis zu der aktuellen Erscheinungsweise der Bilder bedeuten, welches durchaus

91 Zu Seitenzählungsangaben, die mit „FH“ gekennzeichnet sind, vgl. Erklärungen im $\rightarrow$ Literaturverzeichnis.

92 Hier adaptiere ich Hageners Argumentation für den metafilmischen Diskurs. Zur Distinktion: Bei Hageners Analyse (2008) sind es räumlich getrennte Orte innerhalb einer filmischen Fiktion, die vor allem narrativ auf diese Weise aufeinander bezogen werden. „Moreover, the graphic set-up alludes to the playful shifting of distance and proximity in another more oblique way as the split screen allegorises the spectatorial situation in the cinema which is similarly characterised by an oscillation between presence and absence, between proximity and distance“ (Hagener 2008: 2 FH). 
medienhistoriografische Implikationen zeitigen kann. ${ }^{94}$ In Abwandlung der Überlegungen Hageners kann man insofern formulieren, dass über die Bezugsetzung von einem Filmbild zu einem anderen die medienreferenzielle Ebene eingezogen wird, über die im speziellen Falle einer Restaurierungsdokumentation mittels der mehrschichtigen Bedeutungsdimensionen der Bilder der digitale Fortschritt dem Zuschauer vermittelt wird. Dies hat bereits in meinen konkreten Analysen entsprechender Beispiele etwa in $\rightarrow$ Kap. 4 oder $\rightarrow$ Kap. 7 seine Umsetzung gefunden.

Um die wirkungsästhetische Dimension methodologisch und in Fragen dispositiver Anordnungen einzuordnen, wird zudem an dieser Stelle der Begriff des vergleichenden Sehens in seiner Funktion für meine Argumentation kurz näher beleuchtet.

Nicht zuletzt der Kunsthistoriker Heinrich Dilly reflektiert den von Heinrich Wölfflin profilierten Begriff (Dilly 1995: 41), der sich mit den methodologischen Konsequenzen des Einsatzes von Diaprojektoren in der kunstund bildwissenschaftlichen Forschung - sozusagen einer wissenschaftshistorischen Variation eines filmbildlichen Split Screen - auseinandersetzt. ${ }^{95}$ Wölfflins Leistung habe für die kunsthistorische Forschung darin bestanden, "daß er dem ersten Projektor einen zweiten beistellte und somit jeweils zwei an die Wand geworfene Reproduktionen miteinander verglich" (Dilly 1995: 41) ${ }^{96}$

So lassen sich dispositivische Implikationen von Bildkonfigurationen des Vergleichens präzisieren und schließlich methodische Parameter für meine Studie ableiten: In diesem Sinne erscheinen Wölfflins respektive Dillys Überlegungen als historische Ausformulierungen meines Konzeptes der Mise en Relation avant la lettre. Interessant ist dies deshalb, da vor allem Dilly dann die Konsequenzen für die Disziplin und Arbeitsweise der Kunstgeschichte reflektiert.

Seine Aufarbeitung beginnt mit Verfahren der öffentlichen Projektion von Bildern mit erkenntnisleitendem Ansinnen:188o stellte bereits Bruno in seinem Karlsruher Hörsaal den ersten kunsthistorischen Projektor auf (Dilly 1995: 39). Nachfolger Grimm rechtfertigt die Projektion von Bildern im Hörsaal nicht nur aufgrund der gleichzeitigen und gleichmäßigen Anschaulichkeit, sondern

94 „The strategy of negotiating one medium (telephone) via another (cinema) is telling, as it provides the audience with a model for making sense of technological shifts" (Hagener 2008: 3).

95 Vgl. grundsätzlich zum bildwissenschaftlichen Diskurs um Differenzbestimmungen Böhm (2001/1994: bes. 29 ff.); zudem zu Aspekten des vergleichenden Sehens Bader/ Gaier/Wolf (2010).

96 Zur geistesgeschichtlichen Einordnung von Dilly auch mit Blick auf heutige digitale, postfotografische Bildproduktionen vgl. Schröter (2004b). 
auch methodologisch (Dilly 1995: 39). So entstehen etwa ästhetische Versuchsreihen, wodurch sich stilistische Kontextualisierungen über die Bilderrelationen eröffnen. Die Möglichkeiten von Collagen erlauben Denkspiele, das Format der Anordnung erhält zunehmend Bedeutung (Dilly 1995: 40). Solche bildlichen Montageexperimente bedingen ästhetische Argumentationen, Kontextualisierungen erfolgen über Formen und Stil und nicht nur über sozialhistorische Hintergründe. Neue Ordnungssysteme werden über die dispositivische Anordnung der Relationierung möglich. Nach Dilly erfolgt durch neue Reproduktionen ${ }^{97}$ eine Monumentalisierung („Purifikation“) der Kunstwerke, die einer Dekontextualisierung nahekomme: „Großes noch größer erscheinen zu lassen“ (Dilly 1995: 41). Zentral an diesen Ausführungen ist für mich die Beschreibung der erinnerungspolitischen Wirkung von solchen Bildanordnungen, die dann Kunst- oder Mediengeschichte nach neuen Ordnungsund Erlebnissystemen vermitteln:

Das geradezu sakrale Erlebnisangebot enthält nämlich immer auch ein großes Enttäuschungsrisiko. Denn die makellos immateriellen Reproduktionen bleiben, wie Herman Grimm bereits 1893 feststellte, besser im Gedächtnis haften als die Originale (Dilly 1995: 42).

Aus einer dispositivkritischen Perspektive sind die möglichen ideologisch und historiografisch wirksamen Implikationen solcher Bildkonfigurationen - im Modus der Reproduktion - nachdrücklich zu reflektieren.

Schröter (2004b) hat die Ausführungen von Dilly mit den Möglichkeiten postfotografischer Bildproduktion und deren Zirkulation und Deterritorialisierung zusammengebracht. Seine Überlegungen verbindet Schröter mit dem Begriff des „imaginären Museums“ von André Malraux (2012/1965); einem Begriff, der seinerseits bereits in gedanklichem Anschluss an Walter Benjamin zur technischen Reproduktion von Kunstwerken entwickelt wurde.

Vor dem Hintergrund dieses breiten und methodisch vielfältigen Diskursfeldes (Bader/Gaier/Wolf 2010: 14) sind die im Kontext digitaler Filmreprisen zirkulierenden vergleichenden Bildformationen als grundlegende Kulturtechnik eines erinnerungspolitisch wirksamen Denkens und Handelns zu betrachten; dies in der besonderen Ausprägung als phänomenales Erlebnis unter den Vorzeichen kulturindustrieller Formierungen, konstituiert von der Mise en Relation zeitlicher Verhältnisse.

97 Zur erkenntnisbildenden Dimension von (wissenschaftlich-performativen) Dispositiven schreibt Dilly in diesem Zusammenhang: „Darin liegt wohl die Faszination kunsthistorischer Vorträge: Was auch immer gesagt wird, jede Reproduktion hat ihre, wenn auch nur auf einen allerersten Moment konzentrierte Epiphanie“ (Dilly 1995: 42). 


\subsection{Erlebte Mise en Relation. Vergleichendes Hören. THE LODGER (1927), Moroder's Metropolis (1984), LE VOYAGE DANS LA LUNE (1902), VARIETÉ (1925)}

Was für das vergleichende Sehen gilt, kann entsprechend - da man es hier mit audiovisuellen Bildern zu tun hat - auch für die Mise en Relation auf der auditiven Ebene modelliert werden. In diesem Fall handelt es sich dann etwa um auditive Begleitungen und Präsentationsformen, die eine Mise en Relation konstituieren (vgl. $\rightarrow$ auch Kap. 5 zum Beispiel der Re-Edition von LumièreFilmen). Im Dienste einer Konsekration kann der Glaube als Funktionsprinzip des (ökonomischen) Mehrwerts, der dem editierten Werk zugeschrieben werden soll, als ästhetische Strategie und als Effekt über die Tonspur kreiert werden. Dies ist eine gängige Praxis der Aktualisierung von Werken im Zeichen einer zeitgenössischen Popkultur.98

Für den hier diskutierten Gegenstandsbereich ist zu bemerken, dass die Medientransition in die digitale Domäne sich bei der Tonwiedergabe im Kino und auch in der Restaurierung wesentlich unbemerkter vollzogen hat als auf der visuellen Ebene. Interessanterweise verliefen die restaurationsethischen Debatten mit Blick auf die Transition des Tons insgesamt weitaus weniger folgenreich und emotional, als es beim Filmbild der Fall war und ist. Dennoch gab und gibt es Ausnahmen, die die Regel bestätigen: Ein exponiertes Beispiel liefert, wie es Leo Enticknap (2004) in seiner methodologisch argumentierenden Analyse für die Folgen in der Filmwissenschaft beschrieben hat, die Restaurierung von Vertigo in der (Re-)Konstruktion von Tonspuren (F. Heller 2015: 114 f. bzw. Enticknap 2004) ${ }^{99}$

Stummfilme und ihre Musikbegleitung sind die deutlichsten Beispiele für Praktiken der Aktualisierung von Filmen und für die mittelbar historiografisch

98 Dass Musikbegleitung zum einen als Spektakel, aber auch auf der Metaebene zur Konsekration und kulturellen Aufwertung eines Filmes beitragen kann, hat Hansjörg Pauli (1981) eindrücklich für die frühe Phase der Kino- und Filmgeschichte in seiner Studie beschrieben.

99 Die von Robert Harris und James Katz im Auftrag von Universal durchgeführte Restaurierung wurde 1996 abgeschlossen. Generelles Ziel war laut Harris/Katz, den Film an die zwischenzeitlich neu entwickelten technischen Bild- und Tonstandards in Produktion und Projektion anzupassen. Hintergrund der Überarbeitung war unter anderem der ursprüngliche Ton und Sound im nur einkanaligen Monosystem. Das Resultat der Restaurierung von Harris/Katz stellt nun eine Bearbeitung dar, in der eine aus den ursprünglich vorhandenen Materialien dekonstruierte und neu synthetisierte sowie extrapolierte Ton- und Soundmischung mit Sechskanaltechnik vorgenommen wurde. Leo Enticknap (2004) hat sich detailliert kritisch mit den daraus folgenden Transformationen auseinandergesetzt. 
wirksame Formierung ihrer Wahrnehmung in der Geschichte. ${ }^{100}$ Beispielhaft kann dies für Hitchcocks Werk gelten, bei dessen Reprise man eben nicht nur von einem ,vergleichenden Sehen', sondern, wie exemplarisch bei der Edition aus dem Jahr 2012 von THE LODGER (1927) auszumachen, auch von einem, vergleichenden Hören' gesprochen werden kann. So nennt Ivan Radford (2012) in einem Wortspiel den neuen Score von 2012 des Komponisten Nitin Sawhney zu The LODGER, der auf der Blu-Ray-Disc editiert wurde, „post-herrmodern“. Radford wählt diese Formulierung, weil sich in der Musik von Sawhney viele Anspielungen auf Bernard Herrmanns Kompositionen für Hitchcock-Klassiker aus den 1950er und 196oer Jahren finden (Radford 2012). Der Soundtrack von Nitin Sawhney ist auf der 2012 herausgebrachten Blu-Ray-Disc-Edition auswählbar und sogar als Extra-CD der Edition beigelegt. Generell galt für die in Auftrag gegebenen Neukompositionen im Rahmen des öffentlich breit rezipierten Restaurierungsprojekts von Hitchcocks Stummfilmen unter dem Titel HitchCOCK 9:

All three [composers, Sawhney, Cohen, Davies; FH] have named Bernard Herrmann, whose music was integral to Hitchcock's late masterpieces, as an inspiration and the results promise at the very least to reveal connections between the silent films and the better known works that followed (Miller 2011).

Damit ist strukturell in den Kompositionen schon angelegt, dass Rezipienten diese beim Hören mit der vermutlich bekannten Begleitmusik späterer Hitchcock-Filme in Beziehung setzen. Der Filmzuschauer kann so zur Instanz der auditiv erlebten filmhistoriografischen Mise en Relation werden. ${ }^{101}$

100 Vgl. Pauli (1981) grundsätzlich zur historischen Entwicklung, den Aufführungspraktiken und den jeweiligen Musikstücken im Vaudeville-Theater, Zirkus und (später) Kinosälen etwa unter anderem zur Etablierung des ,Manns am Klavier' als verbreitete Form der Filmbegleitung (etwa 68 ff.). Vgl. darüber hinaus mit Blick auf die jüngere Medienumgebung (DVD etc.) den Sammelband von Donnelly/Wallengren (2016) mit zahlreichen Fallbeispielen.

101 Im Kino wie zu Hause mag bei der Sichtung des Films THE Lodge R dieser Soundtrack Befremden hervorrufen, da etwa in einer Schlüsselszene - der Liebesszene zwischen Daisy und dem Mieter - die Zweideutigkeit der Situation über gesungene Liedtexte gedoppelt wird; die Annäherung und zugleich evozierte Gewalt wird in einem Duett besungen: „Blue eyes as cold as ice, cut through me like a knife“. Die plötzliche Wahrnehmung von Gesang bricht mit dem herkömmlichen und bis dahin nur vom instrumentalen Soundtrack etablierten Rezeptionsmodus: „[T] he ambition of the curators is to give contemporary composers a free hand in producing soundtracks. [...] There is room for invention" (Miller 2011). 
Der bekannte Stummfilmpianist, Filmemacher und Autor Neil Brand,102 unter anderem 2012 Komponist des neuen Scores von B LACKMAIL (und zahlreicher anderer Stummfilmscores wie etwa für Chaplins The Gold RusH [1925] auf der MK2/Warner-Home-Video-DVD-Edition aus dem Jahre 2003, vgl. auch unten) hat die Funktion seiner Musik bei Stummfilmbegleitungen damit beschrieben, dass er nicht historisierend spielen würde. Generell gelte für ihn: „There is no definitive score“. Vielmehr folge er dem Prinzip, einen Mix zu finden, der die Distanz zu der vielleicht fremden Ästhetik oder Erzählweise der Filme für ein heutiges Publikum zu überbrücken suche, die Filme aber in ihren ästhetischen Eigenheiten ernst nehme. Damit kommt Brand Kesslers Konzept zum Wiederaufführungsdispositiv historischer Filme heute nicht nur begrifflich, sondern auch in der Funktions- und Wirkungsweise nahe: Bridging the gap, marking the difference (vgl. hierzu meine Ausführungen zum Begriff des Performativen in dieser Studie in $\rightarrow$ Kap. 5).

Eines der bekanntesten Beispiele eines Versuchs, über die musikalische Begleitung eine solche aktualisierende Brückenwirkung im Zusammenspiel mit Stummfilmästhetik zu schaffen, stellt Giorgio Moroders Version von метropolis dar, die bewusst die Aktualisierung der filmischen Bildwahrnehmung über zum Produktionszeitpunkt aktuelle, zeitgenössische Popkultur gesucht hat. ${ }^{103}$ Dies macht diesen Fall aus heutiger Perspektive zu einem sehr anschaulichen Beispiel für die (erlebbare) Historizität von musikalischer ,Kuratierung. Moroder unterlegte eine geschnittene und nachkolorierte Version von Fritz Langs Metropolis mit Musik der Stars der 198oer wie Freddie Mercury, Pat Benatar, Adam Ant, Bonnie Tyler, Loverboy, Billy Squier.

Das Werk kam erst 2011 unter dem Titel Giorgio Moroder PRESEnTs: Metropolis auf DVD wieder in Umlauf, da es bis dahin rechtliche Probleme um die Musiklizenzen gab. Auf der DVD von Kino Lorber wird die ästhetischakustische Aktualisierungspolitik Mordoders mit den folgenden Worten beschrieben:

In 1984, Academy Award-winning composer Giorgio Moroder (MidnIG HT EXPRESS, FLAS HDANCE) introduced Fritz Lang's science fiction epic METROPOLIS to a new generation of moviegoers. Working in collaboration with film archives around the world, he supervised a special reconstruction of the film, with color tinting, fewer inter-titles, and newly restored footage. [...] Unavailable on home

102 Vgl. http://www.neilbrand.com/ (11.10.2016). Neil Brand ist darüber hinaus Autor von Radiostücken, Drehbüchern sowie vor allem von einem Buch mit dem Titel Dramatic Notes. Foregrounding Music in the Dramatic Experience (1998). Vgl. weiterführend zur künstlerischen Arbeitsweise von Neil Brand: Wallengren (2016).

103 Weiterführend zum zeitgenössischen Produktionskontext sowie zur detaillierten Analyse des Scores von Moroder vgl. Smith (2016). 
video for more than two decades, Kino Classics is now proud to revive the 1984 edition of Metropolis, an historic achievement in film restoration (Klappentext, Kino Lorber 2011). ${ }^{104}$

Die DVD von 2011 gerät zu einem Zeugnis der Vorläufigkeit von ,restaurativen“ Versionen $^{105}$ als ein Cluster, dessen Historizität sich vor allem über die Musik der 1980er Jahre vermittelt. Dies verbindet sich mit einer Re-Autorisierung des Werks mit Fokus auf den Komponisten, der eine persönliche, signierte Nachricht der Box beilegt ${ }^{106}$ - immer das Ziel hervorhebend, den Film für neue Zuschauer bekannt machen zu wollen („introducing“). Dabei erscheint interessanterweise seine Version von Mетropolis zusätzlich als Zeugnis digitaler Tontechnikentwicklung - so die paratextuelle Auteur-orientierte Selbst-Stilisierung: „I should also note that my score for MEtropolis was one of the first uses of digital recording of music, which has become the standard today."

Moroder ordnet sich selbst in die philologische Überlieferungsgeschichte des Films ein und kommentiert implizit die Clusterbildung: Seit 1984 sei der Film 2002 und dann noch einmal 2010 restauriert worden, seine Version sei lange von der Zirkulation ausgenommen gewesen - aufgrund der oben genannten rechtlichen Probleme.

Dabei gilt es zu beachten, dass der Begriff der ,Restaurierung' hier von Moroder selbst benutzt wird. Unter restaurierungsethischen Gesichtspunkten gibt es gerade in Diskursen aus dem Archivfeld große Vorbehalte dieser Version gegenüber. ${ }^{107}$ Dennoch bleibt sie ein interessantes Phänomen und Zeugnis, das heute die Vorläufigkeit und Zeitgebundenheit von auditiven Aktualisierungspraktiken veranschaulicht. Die Historizität dieser Version wird auf höchst subjektivierende Weise auf der auditiven Ebene erfahrbar,

104 Vgl. auch Kino Lorber. Website. Giongio Moroder's Metropolis ( $\rightarrow$ Literaturverzeichnis).

105 Die Edition im Jahr 2011 von Moroders Film wirbt damit, materialästhetisch auf der visuellen Ebene ein ,authentisches‘ Zeugnis von Moroders Version aus den 1980er Jahren zu sein: „Rather than substitute digitally enhanced footage from one of the restorations that have occurred in the 27 years since the release of Moroder's Metropolis, Kino Classics has chosen to present the film exactly as it appeared in 1984, mastered from an archival 35mm print" (Kino Lorber. Website. Giorgio Moroders Metropolis; $\rightarrow$ Literaturverzeichnis).

106 Mittlerweile hat sich - hierzu sprichwörtlich sehr passend - im digitalen Vertriebskontext auch das Label ,Signature Edition' gebildet.

107 Zur kritischen Sichtweise aus Archivperspektive von Moroders Version vgl. Bromberg (2012: 12). 
gerade weil die verwendeten Popklassiker stilistisch erkennbar die 198oer Jahre evozieren. Im heutigen zeitlichen Abstand können die bekannten Songs darüber hinaus für verschiedene Generationen von Zuschauern - je nach Alter in unterschiedlicher Form und Intensität - zu einem Erfahrungshorizont zwischen präsentischem audiovisuellem Erlebnis, Wiedererkennen (des Films, der Musik) und Erinnerung in einer autobiografischen Zeitdimension führen; einem Erlebensmodus, der eine auf die eigene Lebenszeit bezogene Mise en Relation - der eigenen Person damals und heute - sinnlich-rhythmisch profiliert. ${ }^{108}$

Ein jüngeres, etwas anders gelagertes Beispiel ist die Re-Edition und Reprise in der Kinovorführung von Georges Méliès LE vOYAGE DANS LA LUNE (vgl. hierzu auch die Analyse der DVD-Blu-Ray-Disc-Edition in $\rightarrow$ Kap. 6). Die Restaurierung aus dem Jahre 2011 wurde nicht nur auf dem Filmfestival in Cannes gezeigt, sondern darüber hinaus auch in einer interessanten Programmierung auf dem Festival Il Cinema Ritrovato in Bologna. Als Teil des Eröffnungsabends im Kontext der Vorführung von Murnaus Nosferatu (1922) als Hauptfilm wurde LE VOYAGE DANS LA LUNE (1902) zunächst mit der modernen, mit elektronischen Elementen spielenden Filmmusik von AIR gezeigt. Diese Musikbegleitung kann aus heutiger Sicht auf den in den Bildern angelegten Science-Fiction-Inhalt des Films verstärkend wirken.

Der Film wurde aber dann darüber hinaus im Vergleich ein weiteres Mal in einer Orchesterbegleitung des Teatro Communale di Bologna unter der Leitung von Timothy Brock nach der Operette von Jacques Offenbach präsentiert. Dies ermöglichte einen Vergleich, in dem die Konditionierung der Zuschauer durch heutige Konventionen der Stummfilmbegleitung auf die Probe gestellt wurde. Die Orchesterversion entsprach hier einer traditionelleren, da ritualisierten musikalischen Begleitung. Insbesondere auch über die performative Gegenüberstellung der aufeinanderfolgenden Präsentationen wurde die Fremdartigkeit, da mit den Konventionen brechend, des neuen, elektronischen Scores von Air hervorgehoben. ${ }^{109}$

An dieser Stelle bietet es sich an, das bereits in Überlegungen zum Performativen (vgl. $\rightarrow$ Kap. 5) dargestellte Konzept von Pescetelli (2010), Filmrestaurierung

108 Zur Erinnerung: Mit einer Abwandlung von Barbara Klinger (2006a: bes. 180) - die sich allerdings mit dem Phänomen des Mehrfachschauens ein und desselben Films auseinandersetzt - sind Formen der autobiografischen, memophänomenalen Einverleibung bereits in $\rightarrow$ Kap. 6 thematisiert worden.

109 Ein paar Monate später im selben Jahr wurde im Rahmen des Festivals Le Giornate del Cinema Muto in Pordenone der Film nur mit dem neuen Score gespielt, wodurch er weniger zur Disposition gestellt schien. 
unter den Vorzeichen einer Aufführung mit Orchesterbegleitung als ereignishaftes Spektakel zu begreifen, im Zeichen des vergleichenden Rezeptionsmodus zu adaptieren: Hier war nicht allein der neue Score von AIR das Spektakel, sondern der programmierte Vergleich, der mit der Qualität des Liveevents verbunden wurde, stellte die Attraktion dar. Damit wurde aber auch die Variabilität der Aufführungsformen von historischen Filmen ausgestellt..10 Mit anderen Worten, der performative Aspekt von früher Filmgeschichte vermittelte einen Unterhaltungsmodus, der sich aus der Varianz der Dimension des Historischen speiste.

Eine Variante dieser Problematik ist der Fall der Restaurierung und ReEdition von Ewald André Duponts VARIETÉ (1925). Der Fall ist deshalb so lehrreich, weil er auf die memopolitischen Implikationen unterschiedlicher diskursiver Frameworks und Dispositive zu verweisen scheint, die unter Umständen durchaus zu äußerst emotionalen Reaktionen führen können (vgl. hierzu ähnlich das Ende von $\rightarrow$ Kap. 6). Die Restaurierung des Films Varieté hatte 2015 auf der Berlinale Premiere - mit der musikalischen Begleitung war das im Zirkus-Vaudeville-Stil zu verortende britische Trio The Tiger Lillies beauftragt. Wie die verantwortliche Restauratorin der Friedrich-WilhelmMurnau-Stiftung während ihrer Masterclass 2015 am Stummfilmfestival Le Giornate del Cinema Muto berichtete, waren die Reaktionen auf die Aufführung durchaus positiv. Anke Wilkening stellte die These auf, dass dies wohl daran lag, dass Besucher nicht nur primär wegen des Films als Attraktion und Spektakel gekommen seien, sondern wohl auch und vor allem wegen der Tiger Lillies, wobei der Film dann als Vehikel der Livemusik gesehen worden sei. Diese Gewichtung kehrte sich allerdings um, als die Restaurierung des Films für den Heimvideomarkt auf DVD von NFP/Friedrich-Wilhelm-MurnauStiftung herausgebracht wurde - mit ebenjener Musikbegleitung. Diese ist zum einen gekennzeichnet von einem Falsettgesang, zum anderen gibt es auch gesungene Textzeilen, die zum Teil die Handlung vorwegnehmen und damit in den Spannungsaufbau und den Rhythmus der Narration des Films eingreifen.

Bei Erscheinen der DVD - so berichtete Wilkening - habe sie extrem negative Reaktionen und Beschwerden über diese Musik erhalten, die äußerst emotional unter anderem im Internet und entsprechenden Foren ausgetragen wurden.

Dispositivisch wurde die in den Vordergrund rückende Wahrnehmung der Musik dadurch verstärkt, dass der Hauptfilm nur mit der Musik der Tiger Lillies

110 Zur Geschichte und zu den historischen Bedingungen der jeweiligen Wahl von Stummfilmbegleitungen vgl. Pauli (1981). 
anzuwählen ist. Allerdings - darauf wies Wilkening hin - ist unter ExTRAs beziehungsweise BonUs die US-Version des Films auswählbar, die mit einer eher den heutigen Stummfilmrezeptions-Gewohnheiten entsprechenden Kinoorgelbegleitung versehen ist.

Vor dem Hintergrund der von Wilkening formulierten Überlegungen bleibt so die interessante Beobachtung einer Verschiebung des Attraktionswertes der Musikbegleitung in unterschiedlichen Dispositiven: vom Mehrwert hin zum Makel und Anstößigen. Insbesondere im Kontext der sammlungsaffinen, cinephilen Aneignung der restaurierten Version für die Rezeption zu Hause artikulierte sich in der ablehnenden, emotionalen Reaktion implizit die Vorstellung eines Werks in einer historischen Integrität, die sich wohl unter anderem mit dem ungebrochenen Genuss des Dramas der erzählten (Mord-)Geschichte verband. Die Musik mit gesungenen Textzeilen galt als zu dominant angesichts der Bilder. So ist letztendlich die Diskussion um VARIETÉ meines Erachtens ein eindrückliches Zeugnis für die unterschiedlichen historiografischen Wirkungsweisen verschiedener Dispositive und ihrer jeweiligen diskursiven, ökonomischen oder sinnlichen Mehrwertversprechen. Umso mehr gilt: Die momentane Mise en Relation beeinflusst die Wahrnehmung des Films, was wiederum - im Kontext der Reprise eines Films mit zugeschriebenem Alters- oder Erinnerungswert - zugleich eine sinnliche Formierung von Historizität bedeutet. ${ }^{111}$

111 Weiterführende interessante Beispiele von Ausprägungen der Mise en Relation stellen in diesem Kontext etwa digitale Editionen dar, in denen Stumm- und Tonfilmfassungen von ein und demselben Film aus heutiger Sicht in einem bestimmten Verhältnis zueinander veröffentlicht werden. Bemerkenswert ist hier, dass meist die eigentlich historisch früheren Stummfilmversionen auf den DVD-Editionen als ,archivarischer Bonus ${ }^{4}$ - da weniger heutigen Sehgewohnheiten entsprechend - den Tonversionen beigegeben werden. Im Fall der DVD-Edition (MK2/Warner Bros. Home 2003) von The Gold RusH mit der Stummfilmfassung aus dem Jahr 1925 und der Tonfilmfassung von 1945 - beide unter der Regie von Charlie Chaplin entstanden - wird tatsächlich die ältere Fassung als ,Archivversion' und Zusatzbeigabe inszeniert. Ähnliche Fälle stellen DVD-Editionen von Alfred Hitchcocks BlackmaIl (1929) dar (u. a. Arthaus 2010). Hier zeigt sich die Pointe, dass sowohl Stummfilm- wie Tonfilmfassung aus demselben Produktionsjahr - eben gerade während der historischen technologischen Umbruchphase - stammen. Zu DVDEditionen von Mehrsprachenversionen vgl. Distelmeyer (2012: 98-99). 


\title{
7.13 Filmgeschichte(n) im Fluss? Dispositivische Konfigurationen am Beispiel von Video-on-Demand (VoD)
}

\author{
„We're moving away from a hard-media society. In the future, there will be \\ only images stored on hard drives, in clouds, and in our memories, as we \\ watch the digital transformation of the cinema“.
}

Dixon 2013, Kap. 1: 16

Im Mittelpunkt dieses $\rightarrow$ Kap. 7 standen bislang Prozesse der Mise en Relation im Horizont von medienhistoriografisch wirksamer Erfahrungsbildung. Im Zuge dessen spielte der Zuschauer/Nutzer/Konsument als Nachvollzugsinstanz eine zentrale Rolle, wobei dispositivische wie filmästhetische Formen der Erinnerungsbildung in ihren medialen Formen und zeitlichen Dimensionen reflektiert wurden.

Dabei stammen die meisten der bisher beschriebenen Beispiele aus den ersten fünfzehn Jahren des 21. Jahrhunderts, welche ich als wichtigen Teil der historischen Phase der Transition hin zu einer digitalen Medienumgebung von Film verstehe. Ich habe zunächst Fälle aus Diskursen, Rezeptions- und Nutzungsformen von DVD und Blu-Ray-Disc erörtert, da ich diese als vorherrschende Trägermedien des historischen Übergangsprozesses vom analogen zum digitalen Film und der impliziten (Film-)Geschichtsbilder begreife.

Aus heutiger Perspektive stellen DVD und Blu-Ray-Disc medienarchäologische Zeugnisse einer spezifischen historischen Phase dar, die Zukunft solcher Trägermedien ist aus der Sicht des Jahres 2019 ungewiss und unabsehbar. Es stellt sich nun die Frage, wie sich meine bisherige Methodik fruchtbar auf andere Dispositive, die Teile einer digitalen Filmkultur sind oder sein werden, übertragen lassen. So bilden zum gegenwärtigen Zeitpunkt vor allem Streaming- und Onlineportale ein heterogenes Feld der Zugriffsformen auf Filme und Filmgeschichte. Dies gilt sowohl für Anbieter aus dem Profit- als auch aus dem Non-Profit-Bereich (etwa Archive, die ihr Material zugänglich machen. Ich haben mich in diesem Zusammenhang bewusst auf stärker kulturindustrielle Kontexte fokussiert). ${ }^{12}$

112 Vgl. zur kritischen Diskussion einiger Plattformen im Zusammenhang mit cinephilen Interessen die Aufstellung von Hagener (2016). Hagener unterscheidet generell bei Plattformen drei Kategorien: 1. Plattformen wie YouTube, Vimeo, Dailymotion, die beitragsfrei sind und sich zu einem großen Teil aus nutzergenerierten Inhalten speisen. 2. Kommerzielle Plattformen, basierend auf einem Abonnementbezahlsystem, mit professionell produzierten Inhalten wie etwa Netflix, Amazon, Mubi, Hulu. 3. Die illegal operierenden Plattformen. Als nichkommerzielle ,webbasierte' Ressourcen nennt Hagener unter anderem archive.org/The Internet Archive wie auch UbuWeb. Vgl. zu einer 
Dazu, ob die DVD beziehungsweise die Blu-Ray-Disc tatsächlich einer Krise unterliegen und generell demnächst als Trägermedien abgelöst werden, gibt es unterschiedliche Positionen und Einschätzungen. ${ }^{113}$ Dixon etwa sieht schon 2013 die DVD und Blu-Ray-Disc als obsolet an (vgl. unten; Dixon 2013, Kap. 1: 2). Aktuell finden sich immer wieder unterschiedliche Formen des Abgesangs auf physische Trägermedien (vgl. hierzu zum europäischen Filmmarkt die Studie European Audiovisual Observatory/Europäische Kommission 2016). ${ }^{114}$

Es kann in einer Untersuchung wie der vorliegenden nicht darum gehen, eine prophetische Prognose für die Zukunft über Medienentwicklungen zu erstellen - oder zu versuchen, in einer derart sich im Fluss befindenden netzwerkartigen und „entropischen“ Medienumgebung (Andrews, n. Hagener 2016: 191) immer neue Phänomene und digitale Dispositive kausallogisch an einige wenige Epistemai fixierend zurückzubinden; ganz zu schweigen davon, der Versuchung nachzugeben, die Digitalisierung in ihren (film-) kulturellen Konsequenzen grundsätzlich, ontologisch oder überzeitlich, bestimmen zu wollen. Man liefe Gefahr, in der analytischen Auseinandersetzung mit konkreten Phänomenen den technologischen Entwicklungen mit all ihren technoimaginären und warenästhetischen Implikationen hinterherzurennen und damit - gerade in dem Versuch, über technizistische Diskurse Erscheinungsformen in einem Ursache-Wirkungs-Modell herleitend

etwas anderen Systematisierung, aber ebenso überblicksartigen Aufstellung von Portalen Krautkrämer/Kirsten/Vonderau (2017).

113 Besonders eindrücklich ist die 2017 zu der Preisverleihung für DVD-Editionen des filmhistorischen Festivals Il Cinema Ritrovato (Bologna, IT) veröffentlichte Stellungnahme zur Situation der sogenannten ,cinephilen' Filmdistributionsfirmen im Zeitalter des Streaming. Der Vizepräsident von Milestone Film \&Video, Dennis Doros, formuliert hier seine Erfahrung und Einschätzung. Die amerikanische Firma Milestone Film \& Video ist spezialisiert auf die Auffindung, Restaurierung und Distribution von Filmen, die in gewöhnlicher Filmgeschichtsschreibung oft als randständig behandelt werden - so geben sie etwa Filme von Regisseurinnen sowie Filme aus LGTBQ-Kontexten heraus. Doros schreibt nun 2017 zu der Arbeit seiner Firma im Zeichen des Streamings: „For the first time in many years, a sizable part of our income is coming from streaming, particularly from Turner Classic Movies' Filmstruck. There are also hundreds of other companies around the world that are seeking the rights to our titles for SVOD [Subscription-Videoon-Demand = Abonnementmodell, FH]. So the writing is definitely on the wall. However, we pride ourselves in doing the years of research for each film, finding the bonus features that put our films into context, and create learning tools for professors and their students to better understand our films. I think most of the companies that do this - such as Criterion, the BFI, Flicker Alley and the rest of our ilk - find this to be the artistic side of film distribution. So there are selfish professional reasons why I hope Blu-ray and DVD distribution will last“ (Doros 2017).

114 Weitere grundsätzliche, exemplarische Onlinebeiträge zu der Diskussion sind etwa Spira (2017) unter dem sprechenden Titel The Death of DVD Will Haunt Us. Vgl. auch ähnliche Artikel von Brew (2017) und Latchem (2015). 
zu erklären - unter Umständen unkritisch die Neuheit erst festzuschreiben. Letztendlich zwingt eine solche Situation insbesondere die medien- und filmwissenschaftlich Forschenden $\mathrm{zu}$ einer noch stärkeren Reflexion und Verzeitlichung ihres Gegenstandes; dieser als Produkt und Faktor einer vorgängigen ästhetischen wie medial dispositivischen (Neu-)Interpretation. Dazu gehört auch eine kontinuierliche selbstkritische Auseinandersetzung mit den methodologischen Implikationen einer sich ständig wandelnden Medienumgebung und ihrer ausgemachten Horizonte.

Ich folge deshalb grundsätzlich Dixons (2013) These - bezüglich der Frage, ob DVD und VoD sich verlässlich und lange in der Zukunft halten -, dass Filme per se phänomenal erhalten bleiben werden; die Plattformen aber - und damit auch die Vielfalt der Dispositive und der anhängigen Diskurse - werden einem ständigen Wandel und der Flüchtigkeit unterliegen. So ist meines Erachtens die Vielfalt und Kopräsenz von Zugangs- und Rezeptionsformen konzeptionell anzunehmen: „Audiences have adjusted to viewing moving images in a variety of different ways" (Dixon 2013, Kap. 1: 12). Allerdings sind bei film- und medienwissenschaftlichen wie vor allem -historischen Fragestellungen die jeweils formierenden Bedingungen der Erscheinungsform jedes digitalisierten Films insbesondere, wenn es sich um historische Reprisen handelt - in Zukunft noch nachhaltiger mitzudenken und die Bedingungen und der Zeitpunkt der Wissensproduktion zu dokumentieren und zu reflektieren.

Bei aller zeitlichen Gebundenheit an sich ständig vollziehende (Medien-) Technologieentwicklungen und an ökonomische Verdrängungskämpfe, die im Austauschverhältnis mit medialen (Nutzungs-)Bedürfnissen seitens der Konsumenten stehen, lassen sich insofern im Rahmen meiner Argumentation folgende grundlegende Tendenzen in einer dezidiert verzeitlichten Perspektive festhalten: Es besteht, wie die Ausführungen in $\rightarrow$ Kap. 7 zeigen, eine Koexistenz verschiedener, zum Teil gegenläufiger Konzepte und Denkmodelle, die sowohl diskursiv, dispositivisch wie ästhetisch in ihrer Relation stets funktional und im Verhältnis zueinander re-konfiguriert und neu ausgehandelt werden. Diese Konfigurationen im Zeichen der digitalen Domäne gehorchen nicht einer Logik oder einer Ideologie (auch wenn sie vom Fortschrittsnarrativ zunächst oberflächlich dominiert erscheinen), vielmehr funktionieren sie als vieldeutige und -schichtige Cluster. Dies gilt grundlegend für das Prinzip der situativ gebundenen Konstruktion von symbolischem Kapital, bei der sowohl kulturelle Autorität als auch der basale (Kunst-)Werkbegriff immer wieder zur Disposition stehen. Die sich ausbildenden momentanen medialen Konfigurationen schreiben sich in je unterschiedliche Erfahrungsdimensionen der Filmbilder ein, konstituieren mit Blick auf den Zuschauer auf diese Weise einen Subjektbegriff, der in seiner leiblichen Präsenz als integraler Bestandteil 
der Konfigurationen zu sehen ist. Wenn in irgendeiner Form der Mehrwert eines Films in seinem historischen Alterswert zum Tragen kommt, dann ist dies als mediale Geschichtsbildmodellierung zu verstehen, die nicht mehr zwischen Fakt, Fiktion und medialer Erfahrung unterscheidet. Mediale Geschichtsbildmodellierungen sind performativ, das wahrnehmende und nutzende Subjekt wird auf unterschiedlichen Ebenen eingebunden; dies unter der besonderen Betonung dessen, dass es immer zum Zusammenspiel der präsentischen Wahrnehmung mit (illusionsbildender) Bewegtbildwahrnehmung kommt. Das zeitigt die Konsequenz, dass der Enunziator Geschichte mit Erinnerungsfunktionen und präsentischen ästhetischen Erlebnisformen des Rezipienten/ Konsumenten verknüpft wird. Dabei muss aus analytischer Sicht immer berücksichtigt werden, was in dem jeweiligen (kulturindustriellen) Kontext situativ die digitale Qualität eines Phänomens in welcher Funktion und mit welchen Konsequenzen vermittelt.

Unter diesen Vorzeichen sind Tendenzen des Video-on-Demand zu systematisieren. Video-on-Demand (VoD) bezeichnet als Oberbegriff die Möglichkeit, über einen Videodienst auf Abruf beziehungsweise Verlangen individuell und zeitunabhängig audiovisuelle Inhalte zu betrachten, indem die digitalen Videodateien etwa zum Herunterladen oder für einen Videostream bereitgehalten werden (Eberle 2007, zit. nach Stegmann 2013: 51). ${ }^{115}$ Dixon konstatiert bereits 2013 aus amerikanischer Perspektive, dass Streaming als die Übertragung und Wiedergabe von Video- beziehungsweise Audiodateien über ein Netzwerk der dominante Modus der Filmdistribution geworden sei (Dixon 2013, Kap. 1: 2). Dies transportiere die Vorstellung von Omnipräsenz von Material - räumlich wie zeitlich. Hagener (2016) greift implizit in diesem Kontext Manovichs (2001) entmystifizierende Sicht digitaler Interaktivität auf, wenn er mit Blick auf Videoplattformen vom "Mythos der Verfügbarkeit“ spricht („myth of availability“, Hagener 2016: 185).

Insbesondere die zeitliche Nähe, die Unmittelbarkeit des Zugriffs - aus jeder alltäglichen Situation heraus - und die Direktheit beschreibt Dixon als entscheidend für die entsprechende Erwartungshaltung der nutzenden $\mathrm{Zu}-$ schauer: „For today's audiences, everything has to be instant“ (Dixon 2013, Kap. 1: 3). Neben dem sofortigen Zugriff etabliert sich eine weitere zeitliche

115 Dixon kommentiert von der technischen Seite her: „The concept of ,streaming“ of course, is nothing new. Early television was essentially streaming video, raw and unprocessed, with plenty of airtime and minimal commercial interruption“ (Dixon 2013, Kap. 1:4). Unter heutigen Bedingungen kommt allerdings die zeitliche und räumliche Ungebundenheit der Abruf- und Rezeptionsmöglichkeiten hinzu, die wesentlich variabler sind als noch bei dem von Dixon zitierten Fernsehen. 
Perspektive, indem verschiedene Zugriffsmöglichkeiten durch immer weiter verknüpfende Vorschläge eine eigene Programmstruktur entwickeln; dies ist vordergründig gekoppelt an bisheriges Sehverhalten von Nutzern eines Streamingangebots, das algorithmisch, das heißt rechnerisch analysiert wird. Das Dispositiv offeriert aufgrund von bisherigen Selektionen eine algorithmisch bestimmte Auswahl, die auf der Rezeptionsebene des Nutzers einen Fluss $(F l o w)^{116}$ von audiovisuellen Angeboten kreiert (dessen Zustandekommen aber letztlich weitgehend opak bleibt).

Bei kommerziellen Anbietern wie Netflix findet sich der nie endende Flussgedanke übersetzt in das gewünschte und suggerierte Konsumverhalten, das in Profit umgemünzt wird. Das zumeist angebotene Abonnement- beziehungsweise Flatratemodell ist auf eine möglichst lange Kundenbindung und Konsumhaltung ausgerichtet (Keiper 2014a, Stegmann 2013:54-56).

Der unter diesen Bedingungen entstehende Programmfluss aus unterschiedlichsten Angeboten ist ein schwer einzusehendes Zuordnungsverfahren. Es wird nicht deutlich, wie ,Personalisierung' eigentlich vom Anbieter definiert wird. ${ }^{117}$ Keiper hat dies mit dem Begriff des "kulturellen Kreisverkehrs" charakterisiert:118 Eine Institution und ein Dispositiv definieren unsere Bedürfnisse nach sinnlicher Erfahrung und Unterhaltung, wir wählen aus und speisen damit wieder das Angebot (Keiper 2014a und 2014b).

116 Weiterführend und ausführlich zum Begriff des Flows im Kontext von Streamingpraktiken und -kontexten vgl. Denecke (2017); in Auseinandersetzung mit seriellen Formen des Fernsehens: Zündel (2017).

117 Diese Form der auf Filme ausgerichteten algorithmisch konstituierten Bedürfnispersonalisierung und -strukturierung ließe sich unter Umständen weiterführend mit Diskursen um ein ,quantisiertes Selbst' verknüpfen, in denen es zunächst - zumindest bei Abend/Fuchs (2016) - um die Vermessung etwa der eigenen körperlichen Verfasstheit (etwa beim Sport o. Ä.) mittels entsprechender Technologien und Programmen zur Datenakquisition geht.

118 Der Filmwissenschaftler David Bordwell hat die Konsequenzen von solchen Zugängen in Hinblick auf eine mögliche historische Filmbildung lakonisch in einem Interview auf den Punkt gebracht: „This is the great paradox of classic film in the age of streaming. If you already know you're a film history buff, it's never been easier to devour title after title. [...] But the gap between ,casual film fan' and ,film history buff' has never been harder - or more expensive - to bridge“ (Bordwell, n. VanDerWerff 2016). Im Anschluss an Diskussionen um Filterblasen (Pariser 2012) und Echokammern des Internets, die versuchen zu vermessen, was man finden möchte, könnte man hier eingedenk von Bordwells Aussage auch von einer filmhistorisch bildenden Filterblase sprechen, die solche Personalisierungen zur Folge haben. Generell zur ,neuen Cinephilie' auch mit Blick auf das Internet und die Blogosphäre vgl. Balcerzak/Sperb (2012). 
Mit Blick auf die Reprise und längere, kontinuierliche Verfügbarkeit von älteren Filmen in solchen Dispositiven schreibt Keiper (2014a):

Obwohl sich VoD immer mehr durchsetzt, ist es sehr stark auf neue Produktionen beschränkt. Eine retrospektive Erfassung des filmischen Erbes ist nicht in Sicht. Allerdings zeigt sich die digitale Ökonomie dieses eine Mal nachhaltiger. Der long tail, also der rentable Abverkauf auch kleiner Lagerbestände, ist in der digitalen Ökonomie mit sehr geringen Kosten verbunden. Sind die Filme erst einmal auf den Servern, ist das Entfernen aufwändiger als der Verbleib - zumindest wenn die Verträge entsprechend konzipiert sind (Keiper 2014a).

Giovanna Fossati beschrieb in diesem Zusammenhang schon 2009 aus der Archivsicht die langfristigen Möglichkeiten der Sichtbarmachung in einem solchen Long-Tail-Ansatz. Sie rekurriert bereits auf den begriffsbestimmenden Aufsatz von Chris Anderson, den dieser unter dem Titel The Long Tail in Wired (2004) veröffentlicht hatte. Fossati beschreibt die Kernaspekte des Modells wie folgt:

The Long Tail model comprises a worldwide distribution system in which the current, relatively small number of mainstream hits - the head of the demand curve (the blockbusters, in film terms) - is substituted by a large number of niches - the tail (the art film, but also the archival film). Thanks to the new ways of on-line distribution this system is becoming economically viable. The need for a large number of people in one place (the film theater) to justify high production and distribution cost, is replaced by the need to satisfy the largest number of individual users spread world-wide with (cheaper) niche products (Fossati 2009: 97).

Insbesondere bei der Wiederaufnahme von historischen Filmen sieht Fossati eine Möglichkeit der Distribution - auch in der Konstitution kleinerer (cinephiler und historisch interessierter) Nischen. ${ }^{119}$ Allerdings sei dies nachhaltig unter der Berücksichtigung von Copyright und Urheberrechtsfragen zu sehen, was sich vor allem auch bei den jeweiligen Katalogen/Angeboten von

119 Vgl. hierzu oben meine Anmerkung zu der Aussage von David Bordwell (n. VanderWerff 2016). Ein weiteres, in diesem Sinne mögliches Diskussionsfeld stellen im Horizont von Onlinevideotheken sogenannte ,Quasiarchive dar, die - unterhalb des Wahrnehmungsschirms der Öffentlichkeit - als invite-only, als geschlossene (Geheim-)Gemeinschaften dezentral und am Rande der Legalität den Zugang zu „einer beachtlichen Zahl filmhistorisch relevanter Werke" bieten (Kirsten/Schmidt 2017: 59). Im Horizont meines Ansatzes könnte auch ein solches Dispositiv auf seine inhärenten (historiografisch wirksamen) Konsekrationsmechanismen untersucht werden, wenn etwa, wie Kirsten/ Schmidt schreiben, „die Netzwerke die Chance [bieten, FH], historisch relevantes Material in qualitativ hochwertigen Digitalisaten nicht nur zu bewahren, sondern ihren Mitgliedern auch ohne Mühe zugänglich zu machen“ (2017: 79). 
Onlinediensten wiederspiegele, die sich auch regional unterscheiden. ${ }^{120}$ So hebt auch Spiegel Online zu Netflix 2016 noch einmal hervor: „Der NetflixKatalog unterscheidet sich von Land zu Land zum Teil erheblich. Das liegt daran, dass Netflix die Rechte für Sendungen für verschiedene Regionen einzeln aushandeln muss" (Spiegel Online 2016).

Für meine Überlegungen sind vor allem Verfahren wichtig, die Keiper (2014b) mit Blick auf Netflix unter der Tagline einer „Vermessung der Seherfahrung" beschreibt. Gerade bei Netflix - aber diese Tendenz lässt sich auch allgemein beobachten - soll mit der suggerierten Personalisierung des Angebots über eine statistische Interpretation auch des Verhaltens anderer, ähnlich eingeschätzter Nutzer eine Typologie von Wünschen und (Konsum-) Bedürfnissen (Keiper 2014b) vorgenommen werden. Algorithmen analysieren und bestimmen Produktion und Angebot, das in den entsprechenden Interfaces in eigens kreierten Programmreihen über symbolische, auf ein Still oder Plakat reduzierte bildhafte Zeichen als Werk offeriert wird. Wirkungsästhetisch soll dies - im Zusammenspiel mit dem Abonnementmodell eine Form des seriellen binge watching beziehungsweise Komaglotzens (u. a. Zündel 2017: 29) hervorrufen, in dem aufgrund von algorithmisch errechneten und dann vorgeschlagenen Serien von Filmen und tatsächlichen seriellen Formaten eine inhaltsindifferente lange Bindung in dem sinnlichen Erlebnis von Bewegtbildern hervorgerufen wird. Mittell (2011) hatte eine ähnliche Rezeptionshaltung schon dem Dispositiv der DVD-Box zugeordnet, die die kulturelle Wertigkeit von editierten Werken beeinflusse (auch weil, so Mittell, dieses Prinzip an den Roman erinnert).

In diesem konkreten Fall der DVD-Box erfolgt eine Konsekration; bei VoD gilt es, dies anders zu perspektivieren. Denn gerade unter dem Vorzeichen der Personalisierung des Angebots verhalten sich die Konsekrationsmechanismen anders als bei einem singulär editierten und zu erwerbenden Werk. Es gibt im VoD-Dispositiv zwar oft noch eine paratextuelle Auteur-Zuschreibung gegebenenfalls auch den Hinweis auf institutionelle Auszeichnungen wie Festivalteilnahmen oder Preise. Aber darüber hinaus wird jedes Werk in der Präsentation - etwa im Falle von Netflix in der Schweiz - vor einem einheitlichen Hintergrund gezeigt; Credits und paratextuelle Informationen sind nur eingeschränkt zu finden. Damit einher geht auch, dass das Menü und entsprechende Bonusmaterialien eines DVD- oder Blu-Ray-Disc-Dispositivs hier mitnichten im Vordergrund stehen. Die eigentlich mögliche, von Distelmeyer (2012) hervorgehobene Flexibilität und die Selektivität digitaler Dispositive

120 Vgl. hierzu weiterführend Op den Kamp (2018) beziehungsweise Fossati (20o9: u. a. 97) sowie einführend zum Geoblocking als (regionale) Kontrolle des Angebots Krautkrämer/ Kirsten/Vonderau (2017: 10). 
werden auf dieser Ebene zurückgenommen. Der Fokus verlagert sich vielmehr auf den Eindruck eines personalisierten Angebots, das auf einer statistischen Hypothese über Wünsche und bisheriges Auswahlverhalten funktioniert (vgl. oben). Dies wird mit einer relationalen Rhetorik verknüpft, die sprachlich eine künstliche Kausallogik vermittelt, die aber die eigentliche Opazität der Verbindungen verschleiert. Es werden in kurzen Ausdrücken Begründungsund sogar Bedürfniszusammenhänge konstruiert wie etwa "title related to“ oder „Der Film könnte Ihnen gefallen, weil .....121 Auf diese Weise werden die sowohl genremäßig wie auch inhaltlich zum Teil recht arbiträr erscheinenden Gruppierungen und Reihungen als vermeintlich ,sinnvoll' vermittelt. Und auch hier setzt der „interaktive Kreisverkehr“, wie Keiper (2014b) es nennt, ein - allerdings unter klaren Vorgaben und (Zeichen-)Reglementierungen: Die Anordnungen von Werken können Ratings berücksichtigen, welche die Nutzer für entsprechende Inhalte abgeben, und beeinflussen - diesmal unter Konformitätsdruck - die Rangordnung sowie die Gruppierung der Inhalte innerhalb der entsprechenden Reihen (Alvino/Basilico 2015). Der Flow des Angebots soll gleichzeitig stets in Bewegung bleiben, die Bindung darf nicht erlahmen, Empfehlungen können sich deshalb regelmäßig - je nach $\mathrm{Zu}-$ griffsform - schon nach wenigen Sekunden ändern. Das Zurücktreten einer Menüstruktur mit Paratexten stellt eine Herausforderung für filmhistorische Angebote dar, vor allem wenn es um kuratierte, historisch kontextualisierende Rahmungen geht. ${ }^{122}$

Als kurzes Zwischenfazit ist festzuhalten: Um der Vielfalt der Ausprägungen von $\mathrm{VoD}^{123}$ unter den momentanen Gegebenheiten gerecht zu werden,

121 Hier gilt mein Dank Joel Singh für Anregungen in der Diskussion.

122 Vgl. zum Verhältnis von DVD und Streaming in Fragen des Begleitmaterials Doros (2017). Zur theoretischen Diskussion der hier anklingenden kuratorischen Prinzipien entweder a. eines Push-Modells, in dem von einer Institution (etwa Firma oder Archiv) etwas kommentiert zugänglich gemacht wird; oder b. eines Pull-Modells, das eher dem Ideal eines individuellen, unmittelbaren und instantanen, spontanen bedürfnisbefriedigenden Zugangs zum Film(inhalt) entspricht, vgl. Fossati/Verhoeff (2007:331). Vgl. darüber hinaus die Beiträge in Paneldiskussionen in den Jahren 2015 und 2016 im Rahmen des Archivfestivals in Bologna, bei denen DVD- und Blu-Ray-Disc-Herausgeber eben genau diese Problematik zur Sprache brachten; vgl. auch die Webseite von alleskino.de, wo die Verantwortlichen damit werben, dass sie Filme historisch kontextualisieren: https://www. alleskino.de/alleskino_die_idee (10.07.2017).

123 Keiper (2014a) notiert nicht zufällig: VoD zerfällt in unzählige Initiativen und Einzelprojekte. Netflix als eine der heutigen (2019) Marktgrößen stellt nur eine der bekanntesten Ausformungen dar, aber in meiner Untersuchung kann es nicht um eine umfängliche Erfassung derVielfalt auch anderer Online-/Streamingportale gehen; vgl. zu einer genaueren Systematisierung der Bezahl- und Finanzierungstrukturen Krautkrämer/Kirsten/ Vonderau (2017: 8 f.). Um weitergehend nur einige Beispiele aus dem archivarischen Kontext zu nennen: European Film Gateway, The Internet Archive; werbefinanziert: 
fokussiere ich die dispositivischen Mehrwertversprechen, die eine vermeintliche Aneignung des Materials über Instantanität, Personalisierung und möglichst lange (emotionale) Bindung des Zuschauers verheißen - wobei dies je nach Zugriffsmodell variiert. Mit anderen Worten, VoD kreiert eigene Formen von Kausalitäten und Serialitäten, die - einer Programmstruktur ähnlich werkübergreifend funktionieren und zur Formierung einer längerfristigen Zuschauerwahrnehmung beitragen. Es entsteht ein Fluss, der allerdings, so die dispositivischen Versprechen, sich auf die Nutzungsvergangenheit jedes einzelnen Nutzers bezieht, zugleich aber von äußeren Begrenzungen wie der regionalen Kataloggestaltung, der zeitlichen Determinierung der Verfügbarkeit von Filmen und der opaken, nicht einsichtigen algorithmisch bestimmten Bedürfnisformierung und -suggestion des Konsumenten abhängig ist. In meinen Augen ist dies eine kulturindustriell höchst ausgeprägte Aus- und Zurichtung einer Wahrnehmungshaltung der Mise en Relation.

Werbeclips des Dispositivs, wie sie bereits im Zusammenhang mit der DVD und Blu-Ray-Disc ausführlich besprochen wurden, formulieren mit sehr ähnlichen ästhetischen Strategien nun diese dispositivischen Mehrwertversprechen. Im Fall von Netflix geschieht dies etwa in einem Clip unter dem auf Bindungen zielenden Titel HoLd ON (entsprechende Lyrics auch im Text des beschwingten begleitenden Songs). ${ }^{124}$ Vor allem wird unter der im Clip prominent auftauchenden Tagline „unlimited stories“ die Vielfältigkeit und unendlich werkübergreifende Dauer des Erlebnisses filmischer Geschichten angepriesen. Es eröffnet sich ein Erlebnisuniversum (aus vielen Einzelgeschichten), auf das man immer und überall Zugriff hat und das damit in vielfältiger Weise die Erfahrung der eigenen Lebenszeit prägt.

Mit Blick auf die Filmgeschichte beschreibt Dixon die raumzeitlichen Dimensionen und zugleich die technoimaginären Implikationen des Instantanen und Omnipräsenten, die mit der Vorstellung von Zukunft identifiziert werden, wie folgt: „Everyone is headed full force into the future, and no one has time for the past" (Dixon 2013, Kap. 1: 22). So macht Dixon auf die Selektionsmechanismen des jeweiligen Katalogs aufmerksam. Zu Netflix stellt er fest:

What about all the classic films that aren't available as streaming video? In essence, they will cease to exist. Netflix is on the fact that most people have no real knowledge of film history, so they'll content themselves with streaming only the most recent and popular films (Dixon 2013, Kap. 1: 5).

YouTube; Abonnement-/Bezahlmodelle: Filmstruck, Mubi, Hulu, Realeyz, Maxdome, Amazon, Alleskino.de u. v. m.

124 Für wertvolle Anmerkungen danke ich hier Andreas Schmunk; weitere Angaben zum Clip $\rightarrow$ Filmverzeichnis. 
Dixon sieht gerade bei Netflix eine nachhaltige Affirmation des ohnehin existierenden Kanons (auch Hagener 2016: 184-185). Dabei spielt die Gewöhnung an den Direktzugriff eine wichtige Rolle, der die zeitliche Schwelle, die früher vor der Befriedigung jedes medialen Konsumbedürfnisses und -zugangs lag, schwinden lässt. Dixon spielt in seinem illustrativen Zitat auf die Vorgeschichte des Video-on-Demand, insbesondere bei Netflix, als einem postalischen Videoversand an:

What will be lost in the process is not only the physical reality of books and DVDs; many titles won't make it to Kindle or streaming video, simply because they're not popular enough. In short, we'll have the ,top ten' classics, and the rest of film history - many superb, remarkable films - will gather dust on the shelf. If you can just click and stream, why wait for the mailman? (Dixon 2013, Kap. 1:6). ${ }^{125}$

Auch wenn es mittlerweile andere Anbieter und Webseiten gibt, über die man zu weniger kanonisierten Werken Zugang erhält (Doros 2017; Krautkrämer/ Kirsten/Vonderau 2017; Hagener 2016), so bleibt doch vor allem Dixons (2013) Argument ${ }^{126}$ ernst zu nehmen, dass eine Institution oder eine Firma erst Geld investieren und damit technische wie inhaltliche Entscheidungen selektiv treffen muss, um einen Film - etwa aus dem Archiv - in die digitale Domäne zu transferieren und damit dort sichtbar und zugänglich zu machen. Es bleibt eine historiografisch wirksame Auswahl und Formierung nach einer Vielzahl von heterogenen Kriterien (ökonomisch, juristisch, kultur- und erinnerungspolitisch, materialästhetisch-quellenphilologisch, technologisch uvm.). Die mit Streaming verbundene Technoimagination des instantanen und omnipräsenten Zugangs verschleiert dies nachdrücklich und nachhaltig.

125 Dixon zitiert hier das folgende Beispiel: „The film (L'IM morTelLe, Robbe-Grillet 1963) is unavailable on DVD, Blu-ray, or even VHS, much less $16 \mathrm{~mm}$; it's available only in $35 \mathrm{~mm}$. To convert it to a digital master and then release it would apparently cost too much money - even with a down-and-dirty transfer - to make the investment back, so the film will remain in limbo, in-accessible to all but the most dedicated historians" (Dixon 2013, Kap. 1: 22). Erneut sei hier auf meine These einer filmhistorisch bildenden Echokammer in Anschluss an Bordwell (n. VanDerWerff 2016) erinnert, die im Horizont des heterogenen Netzwerks von Streamingportalen entsteht: Wer gezielt sucht, findet historische Filme und entsprechendes Material wesentlich schneller und leichter als zuvor; aber die breitere, nichtspezialisierte Sichtbarkeit von historischen Filmen und vor allem auch von verlässlichen und nachzuvollziehenden Kontextualisierungen nimmt ab, beziehungsweise die jeweiligen kanonisierend wirkenden Selektionsmechanismen werden vom Mythos und der Erwartungshaltung der Omnipräsenz von Material überlagert. Auch werden digitale Derivate von Filmen als Analysegegenstand in ihrer Herkunft immer schwerer verortbar.

126 Ähnlich, etwas grundsätzlicher argumentiert Hagener (2016). 
Zugleich ändert sich die Vorstellung vom Erwerb respektive vom tatsächlichen Besitztum eines künstlerischen Objekts, eines Werks - was auch das Verständnis eines (Kunst-)Sammlers beeinflusst: „But as events have proved, with streaming technology, even when you buy something, you don't really own it“" (Dixon 2013, Kap. 1: 8).127 Dies schließt an Diskussionen um DVDs und Blu-Ray-Discs und deren Bedeutung für sammlungsaffine, auf persönliche Archive ausgelegte Cinephilie an und zeigt die Variation von Konsekrationsmechanismen an dieser Stelle (u. a. Wortmann 2010). ${ }^{128}$

Diese Gemengelage um die Zugriffsform über Streaming und den prekären ,Objekt'-Status von Film (Film verstanden als „content“, Cherchi Usai 2012) zeitigt Konsequenzen; dies nicht zuletzt, weil der Gebrauchswert von Filmen in anderen Relationen werkübergreifend nun in dem seriellen Modus, in möglichst extensiver Unterhaltung gesehen wird - im Horizont ästhetischen entertainments als commodity in einer vom Anbieter kreierten Dauer:

,Watch it and forget it' is a reasonable model of consumption. Collectors may want to hold on to their DVDs for a variety of reasons - some sort of fleeting permanence being one of them - but for most viewers, the idea is to watch it now and then move on (Dixon 2013, Kap. 1: 24).

Insbesondere die über diese Form der Filmerfahrung kreierte Serialität impliziert wahrnehmungstheoretische Konsequenzen - gerade mit Blick auf die Erinnerungsaktivität und -praxis des Rezipienten/Konsumenten. ${ }^{129}$ Sowohl für die memotechnische Komponente als auch für die Zeiterfahrung als (über)filmische Dauer im Sinne einer subjektiv erlebten Zeit (Henri Bergson) assoziiert Dixon das Bild von fließendem Wasser. Mit dieser Metapher veranschaulicht er zugleich die Rezeptionshaltung und die Wertschätzung von

127 „An early har-binger of this was Amazon's now famous (and all too obviously ironic) deletion of George Orwell's 1984 from Kindles around the United States when the company discovered it had accidentally uploaded an unauthorized version of the novel“ (Dixon, Kap. 1: 8).

128 Dixon (2013, Kap. 1: 11) macht in diesem Kontext auf die Problematik der nötigen Pflege der digitalen Daten und auf die Ungelöstheit der Probleme digitaler Langzeitsicherung aufmerksam: „[S]torage of digital imagery is a never-ending process of maintenance and upgrading“. Damit spricht er einen wesentlichen, oft übersehenen Kernaspekt beziehungsweise eine Bedingung der Sichtbarkeit von Filmen an, da die nachhaltige Verfügbarkeit in der digitalen Domäne keineswegs gelöst ist und tatsächlich einer aktiveren Strategie zur Sicherung und Archivierung bedarf. Vgl. insbesondere hierzu sowie zu den notwendigen, auch praktischen wie kulturpolitischen Konsequenzen F. Heller (2017).

129 Vgl. zur grundsätzlichen Auseinandersetzung von VoD im Verhältnis zur Serialität des Fernsehens Zündel (2017). 
ästhetischer Erfahrung über VoD:130 „In short, we live in a streaming world where everything, inevitably, will be available like running water - all you have to do is pay the utility bill" (Dixon 2013: 24). ${ }^{131}$ Nicht nur dokumentiert Dixons Bild den kulturindustriellen Gebrauchszusammenhang von Filmen als ästhetischem Erlebnisfluss. Man ist darüber hinaus versucht, dieses Bild mit Heraklit zeitphilosophisch und damit erinnerungspolitisch zu wenden: „Man kann nicht zweimal in denselben Fluss steigen." Aus erinnerungskultureller Perspektive scheint somit VoD eine Mise en Relation zu etablieren, die auf ein ständiges Fließen ausgelegt ist. Bedeutungsgenerierende Konfigurationen manifestieren sich hier nur situativ, sie sind wenig fixiert und stellen damit permanent neue (historische) Zusammenhänge her.

\subsection{VoD und Versionenhaftigkeit. DAs Bоот (1981/1985) als amphibischer Film}

Unter den oben formulierten Vorzeichen und Selektionsmechanismen ist im Kontext dieses $\rightarrow$ Kap. 7 abschließend nach der Bedeutung des für die Filmgeschichte und die entsprechende Geschichtsbildmodellierung konstitutiven Phänomens der Versionenhaftigkeit zu fragen.

Das mögliche Fehlen einer Menüstruktur, die unter Umständen den Zugriff auf unterschiedliche Versionen eines Werkes ermöglicht hätte, impliziert, wie oben mit Rekurs auf Distelmeyer (2012) schon angesprochen, dass Formen der audiovisuellen werkspezifischen Kontextualisierungen zurückgenommen werden: So wird, um wieder eines der populärsten Versionenphänomene der

130 Theoriegeschichtlich ist die Begriffswahl von Dixon angelehnt an Raymond Williams' Begriff vom Flow; der über Zapping von Rezipienten hergestellten (eigenen) Programmstruktur des Fernsehens. Die Metapher des Flusses ist in diesen Zusammenhängen allgegenwärtig, ob als Programmfluss oder auf der Ebene der zirkulierenden Ausbreitung; vgl. Denecke (2017) sowie Hagener/Hediger/Strohmaier (2016). Im letztgenannten Band wird allerdings das Bild des Wassers als Metapher der ausströmenden Zirkulation verwendet. Auch wird das Bild des Flusses für die besondere Flüchtigkeit der Verweildauer des Materials in der Streamingdomäne bemüht.

131 Dies ist auch auf der Ebene der konkreten Selektion für den Katalog zu sehen: „Of course, there are still large gaps - films [...] that haven't entered the streaming era: films that are too marginal or are strictly controlled by their makers; ,orphan' films that have no legal copyright status or no living owners and have thus entered the public domain, making them available to anyone but significantly diminishing their potential for corporate profits" (Dixon 2013: 24). Vgl. weiterführend zum Problem insbesondere von ,Orphans'/ verwaisten Filmen und Copyright - und damit ihrer Sichtbarkeit in der digitalen Domäne Op den Kamp (2018). 
jüngeren Filmgeschichte zu zitieren, etwa auf Netflix/Schweiz im Herbst 2015 von BLAde RunNer nur der Final Cut - dieser aber zumindest über den Titel ausgewiesen, aber ansonsten unkommentiert - zugänglich gemacht. Dem generellen Design und Interface des Anbieters unter- und eingeordnet erfolgte hier nur eine minimale Angabe der Produktionsdaten. Dazu in einer zeitlich limitierten Form: 2017 existieren der FinAL CUT sowie der TheAT RICAL CUT lediglich nur noch als Suchbegriffe in der Maske - ansonsten wird uns eine auf den ersten Blick doch in Thematik und Genre zum Teil überraschende Produktpalette vermeintlich ähnlicher Filme angeboten, zu der nur textsprachlich suggeriert wird, dass sie mit Ridley Scotts Film in einem - wie auch immer gearteten - Verhältnis stünde: „Titles related to“ (Abb. 7·39).

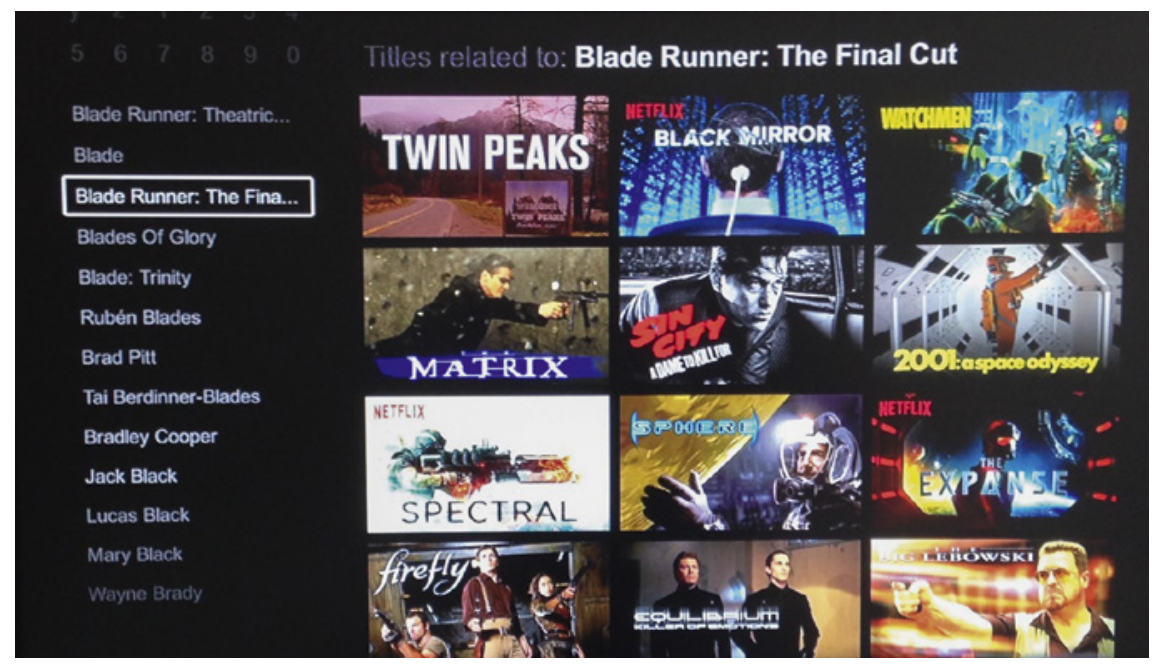

Abb. $7 \cdot 39$

Bei einem schon vom historischen Produktionskontext her clusterhaften, gar als „amphibisch“ zu bezeichnenden Werk wie DAs Bоoт (Kinofassung 1981) von Wolfgang Petersen hat das zur Folge, dass in der Zuschneidung und Nichtkontextualisierung der komplexen Überlieferungs- und Produktionsgeschichte des Films zugleich auch die Wahrnehmung des auf Zeitgeschichte referenzierenden Filminhalts formiert wird.

Zur Erinnerung:132 DAS Воот ist zu sehen als eine "Sonderform des amphibischen Films"; ein Begriff, den der Produzent Günter Rohrbach mit Blick auf die Zusammenarbeit von Film und Fernsehen geprägt hatte (Scherfer 1998:

132 Für Anregungen in der auf das Dispositiv bezogenen Analyse der Versionen von DAS Воот danke ich Petra Uphues. 
218). Das Ergebnis der koproduzierenden Zweckgemeinschaft aus privaten Filmgesellschaften und öffentlich-rechtlichen Fernsehanstalten waren unterschiedliche Versionen: Eine Kinofassung, die 1981 in die Kinos kam, sowie die Version in Form einer TV-Miniserie (erst 1985 drei-, dann 1987 sechsteilig ausgestrahlt). Die Versionen entstanden aus Material, das im selben Produktionsprozess gedreht wurde. Als Veröffentlichung weiterer Versionen folgte zunächst 1997 die Fassung Das Boот. The Director's Cut mit einer Distribution im Kino sowie auf DVD - eine der ersten DVDs in Deutschland überhaupt. 2011 erschien eine Blu-Ray-Disc, über die der Film als JubıLÄUms-EDitıon erneut auf den Markt gebracht wurde (Distelmeyer 2012: 21-23).

2004 wurde auch die TV-Filmfassung als DVD veröffentlicht. Auf zwei DVDs wurden „[f]ast 5 Stunden Spannung und Dramatik pur“ versprochen. Die ursprüngliche TV-Miniserie ist für diese Edition verändert worden. Die einleitende ,Rückblende‘ als Wiederholung am Anfang jeder Folge wurde weggelassen und das Material zu einem Film aneinandergereiht (Relinger 2009: 80). 2014 erschien die TV-Serie in der sechsteiligen Version auf Blu-Ray-Disc (Eurovideo, 2014) - vermarktet unter der Tagline „DAs Воот taucht wieder auf - in voller Länge!“.

Die einzelnen Versionen - die Fernsehserien auf der einen und die Kinofassungen auf der anderen Seite - unterscheiden sich in der narrativen und ästhetischen Struktur grundlegend dahingehend, dass sie - zudem bedingt durch die unterschiedliche Laufzeit und dispositivische Rahmung (TV vs. Kino) - eine alternative erzählerische Dynamik und zeitliche Rhythmik aufweisen (Relinger 2009: 82). Vor allem wird in der Serie inhaltlich-atmosphärisch den Wahrnehmungsveränderungen angesichts der räumlichen Enge an Bord, dem veränderten Raum- und Zeiterleben der Matrosen im U-Boot-Krieg mehr Platz eingeräumt. Durch die Einstreuung von entschleunigenden, handlungsarmen Bildern wird eine nachdenklichere Stimmung erzeugt, als es in der Version für das Kino der Fall ist, die im Vergleich mehr auf die Kriegshandlung ausgelegt ist (Uphues 2016). So beeinflusst die jeweilige Version deutlich die filmische Darstellung und Vermittlung einer historischen Kriegserfahrung, die gerade in Hinblick auf DAs Воот oft in einem dokumentarischen Referenzmodus gelesen und somit in enger Beziehung zur Ereignisgeschichte des Zweiten Weltkriegs wahrgenommen wird (Uziel 20o1).

Auf die Präsentation und Zugangsform über Netflix in der Schweiz bezogen, ist vor diesem Hintergrund festzuhalten, dass diese Formen der sowohl filmwie mittelbar ereignisgeschichtlichen Formierung in keinem entsprechenden Kontext der Versionenhaftigkeit des Films wahrnehmbar wird. ${ }^{133}$ Im Juli 2017

133 Interessant ist in diesem Kontext, dass auf der frei zugänglichen Webseite von Alleskino.de, die explizit mit einem filmhistorisch bewussteren Umgang und der 
etwa bekommt man über die Titelsuchfunktion „Das Boot“ letztendlich die TV-Miniserie angeboten (Abb. 7.40); diese wird paratextuell ausgezeichnet als die Version von 1985: „The original television mini series version of the classic war thriller about a German U-Boat crew's harrowing, deadly mission in World War II“.

\section{Das Boot}

$1985 \quad 12 \quad 1$ Season HD

The original television miniseries version of 7.61 194. the classic war thriller about a German U-Boat

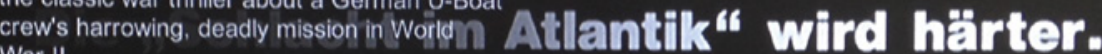
War II.

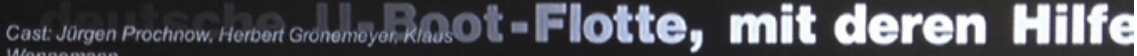
Wennemann Creator: Wolfgang Potorsen
TV Shows, TV Dramas

Play Season 1: Episode 1 คinkschläge erlftten. Immer

More episodes werden die in Celeitzitg

四 Audio and subtitles IIIG englischen Frachtschifie vor

+ Add to My List rern abgesichert. Dennoeh werde ৬ Rate this title anzösischen Atlantlkhẗen aus in neue U-Boote in den Kampf geschiokt.

Abb. 7.40

Erst bei der Anwahl der einzelnen Episoden wird deutlich, dass es sich um die sechsteilige Serienversion handelt (Abb. 7.41). Es ist somit die Version von DAS Bоoт, die die fließende epische Dauer der seriellen Produktion am meisten ausspielt. Die zeitliche Entfaltung passt sich hiermit der Erwartung an den vom Dispositiv gewollten Erlebnisfluss an - bei gleichzeitiger mehrschichtiger

Kontextualisierung der von ihr zugänglich gemachten deutschen Filme wirbt, ausgerechnet in der Selbstbeschreibung in der Rubrik „Über uns“ im Hintergrund ein Filmstill aus DAs Bоот zu sehen ist, das auch textsprachlich als Director's Cut gekennzeichnet wird (zuletzt gesehen am 12.07.2017). Ich begreife dies als eine spezifische Form eines vielschichtigen filmhistorischen Konsekrationsakts, in dem die Selbstdarstellung des Anbieters mit quellenphilologisch geprägtem Bewusstsein, Autorisierungsstrategien und zudem mit der filminhaltlich verhandelten realgeschichtlichen Dimension sowie dem internationalen Erfolg von Petersons Film assoziiert werden soll. 


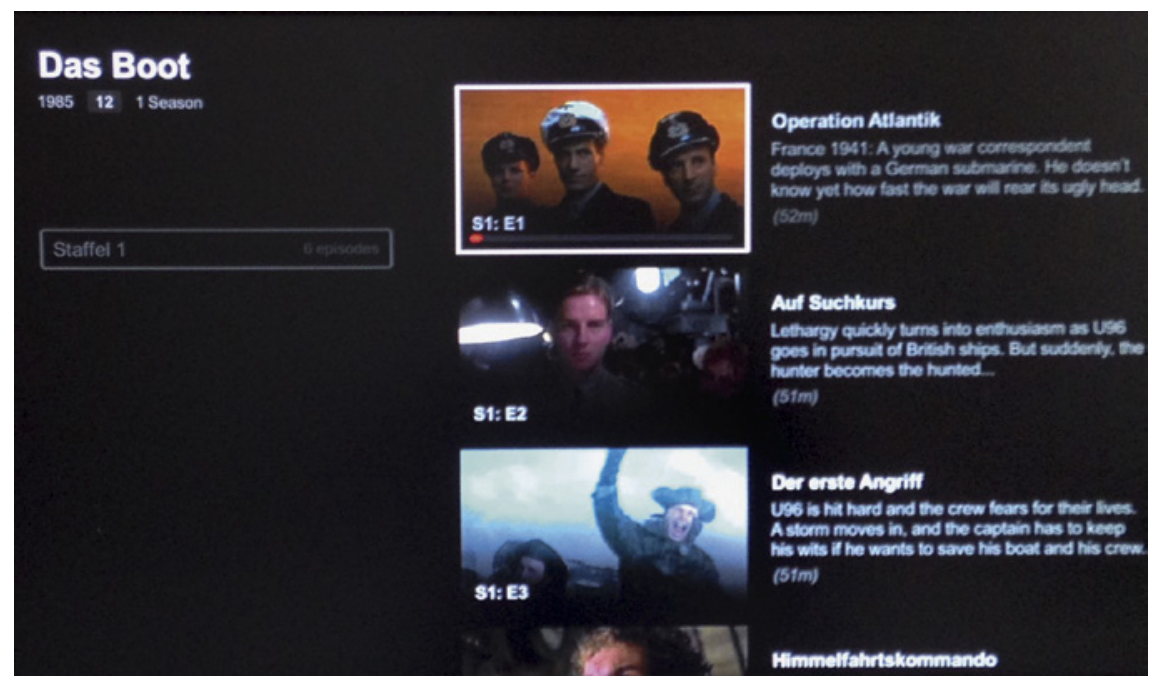

Abb. 7.41

Assoziierung einer bedeutungstragenden, historischen Dimension. Hierbei verweben sich die filmische Metaebene der Überlieferung und deren auf den historischen Status des Werkes ausgerichtete Konsekrationsrhethorik „original“ und „classic“ - mit dem die Erwartung formierenden inhaltlichen Label „Zweiter Weltkriegsthriller“. Zugespitzt könnte man formulieren, dass die auszuwählende Version mit ihren jeweiligen historisierenden Lektüremodi - in der Verwebung von metafilmischem Status und Filminhalt Teil des kontinuierlichen Flusses einer Bewegtbildgegenwart wird. In einem solchen Kontext könnte man von einer Variante der zeitlich personalisierten Geschichtsbildmodellierung sprechen, die den nutzenden Zuschauer vielschichtig - nicht zuletzt auch über die mobilen Zugriffsmöglichkeiten auf VoD - in seiner Lebens- und Alltagsdauer einbindet, funktionalisiert und affiziert.

Das kurz behandelte Beispiel DAs Boot auf Netflix verdeutlicht im vorliegenden Zusammenhang Tendenzen, die man grundsätzlich dispositivisch und erinnerungskulturell einordnen kann: Zum einen suggeriert das Dispositiv Video-on-Demand eine an den Nutzer gebundene Dauer, die vor allem werkübergreifend funktioniert. Die Erinnerungsfunktion des Nutzers wird im Fluss des personalisierten Programms zum Mitkonstituens der seriellen Anordnung von Filmen. Wenn in solchen Kontexten einem Werk dann ein Altersoder Erinnerungswert zugeschrieben wird - in dem Sinne, dass es historisch referenzierend (ob metafilmisch oder ereignisgeschichtlich gelesen) wird dann finden eine Vielzahl von historiografisch wirksamen Formierungen 
statt. Einmal mehr kommt das Zusammenspiel von Erwartung und konkreter Erfahrung zum Tragen. Allerdings sind diese Ausprägungen der zeiträumlichen Mise en Relation nur noch schwer inhaltich in vollem Umfang zu systematisieren.

Dijk (2007) hat in ihrer Reflexion zur Rolle von digitalen Medien für Erinnerungspraktiken und -funktionen gerade auch mit Blick auf die Zugriffsformen von einer googlization von Gedächtnis und Erinnerung gesprochen. Sie stellt in diesem Zusammenhang die Frage nach der Re-Definition des Verhältnisses von Gehirn und Maschine (Dijk 2007:xvi): Die Maschine erkenne Muster und interagiere damit von außen mit unseren Erinnerungen und Handlungen, die unser Selbst konstituierten. Kulturelle und mediale Phänomene verbänden sich so mit persönlichen Erinnerungen und damit mit der Selbstkonstitution. Öffentlich und privat seien als Kategorien nicht mehr voneinander zu trennen: Es finde ein mutual shaping statt; Öffentliches werde verinnerlicht, und umgekehrt werde über persönliche Erinnerungsprozesse kulturelles Gedächtnis affirmativ konstituiert. Dies habe im Horizont der digitalen Medienumgebung einen performativen Charakter (Dijk 2007: 169).

Angesichts dieser Überlegungen haben Bewegtbilder aufgrund ihrer sinnlichen Erlebnisqualitäten sowie ihrer Möglichkeiten zur narrativen Illusionsbildung das besondere Potenzial, sich in die kulturelle Gedächtnisbildung einzuschreiben (Welzer/Moller/Tschuggnall 2002). Bewegtbilder nehmen damit über ihre spezifischen raumzeitlichen Vermittlungsmodi Geschichtsbildmodellierungen vor, in denen Fakt und Fiktion - Reales und Imaginäres - sich jeweils abhängig von der medialen und ästhetischen Konfiguration konstituieren. Dies verbindet sich wirkmächtig mit der Zirkulation und den Zugriffsformen zwischen öffentlicher und privater Gedächtnisbildung. Der sich auf eine privatwirtschaftliche Firma beziehende Begriff der googlization deutet schon darauf hin, dass der gesamte Komplex zudem nicht losgelöst zu sehen ist von den ökomischen Interessen des jeweiligen Dispositivs. ${ }^{134}$ Insofern scheinen kritische filmanalytische Ansätze zu heutigen Formen der Warenästhetik digitaler Dispositive höchst aktuell und notwendig. Dies wird umso anschaulicher, wenn unter den Maßgaben der Interessen von kulturindustriell betriebenen Dispositiven filmische Geschichtsbildmodellierungen - etwa über die Auswahl von Filmen und re-kontextualisierten Filmversionen Erwartungen und Erfahrungen der personalisierten oder interaktiven Mise

134 Vgl. konkret zu Google als Institution, die kulturelle Praktiken des Wissens kreiert, die Studie von Röhle (2010) zum „Google-Komplex“. 
en Relation vermittelt werden. Dies hat eine zeiträumliche Relativierung historischer Wahrnehmungsmodi und damit verbundener historiografisch wirksamer (leiblicher) Erfahrungsbildung des Subjekts zur Folge. So hat das Versprechen der Do-it-yourself-History wahrnehmungstheoretische, darüber hinaus aber auch grundsätzlichere geschichtspolitische Konsequenzen, die stets im Blick behalten werden müssen. So ist mein Fazit vor allem ein methodisches: Diese Aspekte sind weiterhin in jedem gegebenen Kontext in ihrer Funktionsweise kritisch zu hinterfragen. Dies gilt besonders für die Konstitution eines historischen Subjekts angesichts von extrem dynamischen und flüchtigen medialen Konfigurationen, die mit Bewegtbildern arbeiten. Solche sind jeweils sowohl als Erwartungshorizonte, in denen sich Technoimaginationen digitaler Medien spiegeln, als auch als unmittelbare körperliche Erlebnissphären zu konzipieren. 